\title{
Two-Multicast Channel with Confidential
}

\section{Messages}

\author{
Hassan ZivariFard, Matthieu Bloch, Senior Member, IEEE, and Aria Nosratinia, \\ Fellow, IEEE
}

\begin{abstract}
Motivated in part by the problem of secure multicast distributed storage, we analyze secrecy rates for a channel in which two transmitters simultaneously multicast to two receivers in the presence of an eavesdropper. Achievable rates are calculated via extensions of a technique due to Chia and El Gamal and the method of output statistics of random binning. Outer bounds are derived for both the degraded and non-degraded versions of the channel, and examples are provided in which the inner and outer bounds meet. The inner bounds recover known results for the multiple-access wiretap channel, broadcast channel with confidential messages, and the compound MAC channel. An auxiliary result is also produced that derives an inner bound on the minimal randomness necessary to achieve secrecy in multiple-access wiretap channels.
\end{abstract}

\section{INTRODUCTION}

We study the multiuser secure multicast problem (Fig. 1), more specifically, when two transmitters multicast messages securely to two receivers in the presence of an eavesdropper. All senders, receivers, and eavesdropper are at different terminals. This problem is motivated in part by secure access of multiple users to data in a distributed cache, which is a multi-transmitter (multiple-access) multicast scenario [1], [2]. This problem is also equivalent to a compound twostate multiple-access wiretap channel. It has been known [3] that problems involving compound

H. ZivariFard and A. Nosratinia are with Department of Electrical Engineering, The University of Texas at Dallas, Richardson, TX, USA. M. Bloch is with School of Electrical and Computer Engineering, Georgia Institute of Technology, Atlanta, GA, USA. E-mail: hassan@utdallas.edu, matthieu.bloch@ece.gatech.edu, aria@utdallas.edu.

The material in this paper was presented in part at the 55th Annual Allerton Conference on Communication, Control, and Computing, Monticello, IL, September 2017. 


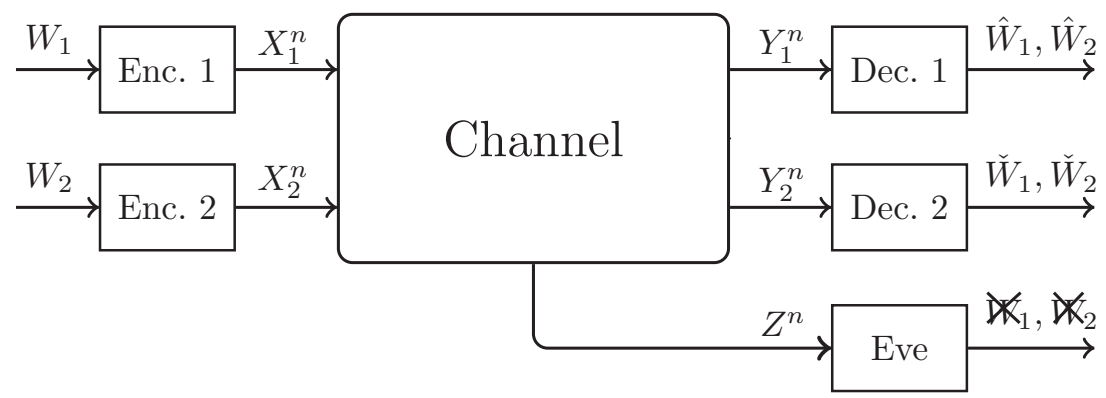

Fig. 1. Two-sender two-receiver channel with an eavesdropper

channels have an equivalent multicast representation, in which the channel to each multicast receiver is equivalent to one of the states of the compound channel 1

This paper takes a two-pronged approach to the analysis of the network mentioned above, producing a number of new results and insights. In Section [II] we present an analysis inspired by the work of Chia and El Gamal [7], which uses Marton coding and indirect decoding (also known as non-unique decoding) [8] to achieve an improved secrecy rate for the transmission of one common message to two receivers that may experience different channel statistics. In extending the method of Chia and El Gamal to multiple transmitters, we introduce a two-level Marton-type coding with associated non-unique decoding.

In Section VI, we employ the method of output statistics of random binning (OSRB) [9] for analyzing the two-transmitter two-receiver problem (see also [10] for a related approach). OSRB analyzes channel coding problems by conversion to a related source coding problem, where it tests achievability by probability approximation rather than counting arguments on typical sets, followed by a reverse conversion to complete the analysis. OSRB is well suited for secrecy problems because secrecy is tightly related to probability approximation. OSRB encoding is purely by random binning and is enabled by (and named after) the following asymptotic result: apply two independent random binning schemes on the same set and take a random sample from the set. The two bin indices corresponding to the random sample are statistically independent as long as binning rates are sufficiently small [9]-[11]. We extend the tools and techniques of OSRB to match the requirements of the two-transmitter multicast problem.

\footnotetext{
${ }^{1}$ The problem studied herein is the secrecy counterpart of the classical problem posed by Ahlswede [4], which proved highly influential for the MAC channel [5] and the interference channel [6].
} 
The different parts of this paper complement each other, producing a more complete picture in the understanding of the problem of multi-transmitter secure multicast. The extension of the method of Chia and El Gamal is utilized to highlight the minimal amount of randomness required to achieve secrecy rates over the multiple-access wiretap channel, and that therein channel prefixing can be replaced with superposition, in a manner reminiscent of Watanabe and Oohama [12] for minimizing the randomness resources for secrecy encoding. The analysis based on OSRB generates strong secrecy, which interestingly has an expression that is a superset of the achievable weak secrecy calculated in the first part. Furthermore, the expression for the strong secrecy region can be greatly simplified via a constraint found in the weak secrecy analysis, highlighting the synergy between the two. More broadly, the developments in these two parts each offer techniques and insights that can potentially be useful in a wider class of problems.

Outer bounds for degraded and non-degraded channels are derived and shown to be tight against inner bounds in some special cases. Part of the results, including the proof of the outer bounds, appeared in an earlier conference version of this paper [13] and are not duplicated here in the interest of brevity.

A brief outline of the related literature is as follows. Multicasting with common information in the presence of an eavesdropper has been studied in [14], [15], deriving inner bounds on secrecy capacity, and in some special cases also deriving secrecy capacity region. Salehkalaibar et al. [14] studied a one-receiver, two-eavesdropper broadcast channel with three degraded message sets. Ekrem and Ulukus [15] studied the transmission of public and confidential messages to two legitimate users, in the presence of an eavesdropper. Benammar and Piantanida [16] calculated the secrecy capacity region of some classes of wiretap broadcast channels.

The MAC wiretap channel has been investigated in [17]-[24]. In [17], a discrete memoryless MAC with confidential messages has been studied that consists of a MAC with generalized feedback [25] where each user's message must be kept confidential from the other. The multiple access wiretap channel [18], [19], [23] consists of a MAC with an additional channel output to an eavesdropper. In [18], [19], achievable rate regions for the secrecy capacity region have been derived. Secrecy in the interference channel and broadcast channel has been studied in [26], where inner and outer bounds for the broadcast channel with confidential messages and the interference channel with confidential messages have been compared. 


\section{PRELIMINARIES}

Throughout this paper, random variables are denoted by capital letters and their realizations by lower case letters. The set of $\epsilon$-strongly jointly typical sequences of length $n$, according to $p_{X, Y}$, is denoted by $\mathcal{T}_{\epsilon}^{(n)}\left(p_{X, Y}\right)$. For convenience in notation, whenever there is no danger of confusion, typicality will reference the random variables rather than the distribution, e.g., $\mathcal{T}_{\epsilon}^{(n)}(X, Y)$. The set of sequences $\left\{x^{n}:\left(x^{n}, y^{n}\right) \in T_{\epsilon}^{(n)}(X, Y)\right\}$ for a fixed $y^{n}$, when the fixed sequence $y^{n}$ is clear from the context, is denoted with the shorthand notation $\mathcal{T}_{\epsilon}^{(n)}(X \mid Y)$. Superscripts denote the dimension of a vector, e.g., $X^{n}$. The integer set $\{1, \ldots, M\}$ is denoted by $\llbracket 1, M \rrbracket$, and $X_{[i: j]}$ indicates the set $\left\{X_{i}, X_{i+1}, \ldots, X_{j}\right\}$. The cardinality of a set is denoted by $|\cdot|$. We utilize the total variation between probability mass functions (pmfs), defined by $\|q-p\|_{1}=\frac{1}{2} \sum_{x}|p-q|$. Following Cuff [27] we use the concept of random pmfs denoted by capital letters (e.g. $P_{X}$ ).

Definition 1. $A\left(M_{1, n}, M_{2, n}, n\right)$ code for the considered model (Fig. 1) consists of the following:

i) Two message sets $\mathcal{W}_{i}=\llbracket 1, M_{i, n} \rrbracket, i=1,2$, from which independent messages $W_{1}$ and $W_{2}$ are drawn uniformly distributed over their respective sets.

ii) Stochastic encoders $f_{i}, i=1,2$, which are specified by conditional probability matrices $f_{i}\left(X_{i}^{n} \mid w_{i}\right)$, where $X_{i}^{n} \in \mathcal{X}_{i}^{n}, w_{i} \in \mathcal{W}_{i}$ are channel inputs and private messages, respectively, and $\sum_{x_{i}^{n}} f_{i}\left(x_{i}^{n} \mid w_{i}\right)=1$. Here, $f_{i}\left(x_{i}^{n} \mid w_{i}\right)$ is the probability of the encoder producing the codeword $x_{i}^{n}$ for the message $w_{i}$.

iii) A decoding function $\phi_{1}: \mathcal{Y}_{1}^{n} \rightarrow \mathcal{W}_{1} \times \mathcal{W}_{2}$ that assigns $\left(\hat{w}_{1}, \hat{w}_{2}\right) \in \llbracket 1, M_{1, n} \rrbracket \times \llbracket 1, M_{2, n} \rrbracket$ to received sequence $y_{1}^{n}$.

iv) A decoding function $\phi_{2}: \mathcal{Y}_{2}^{n} \rightarrow \mathcal{W}_{1} \times \mathcal{W}_{2}$ that assigns $\left(\check{w}_{1}, \check{w}_{2}\right) \in \llbracket 1, M_{1, n} \rrbracket \times \llbracket 1, M_{2, n} \rrbracket$ to received sequence $y_{2}^{n}$.

The probability of error is given by:

$$
P_{e} \triangleq \mathbb{P}\left(\left\{\left(\hat{W}_{1}, \hat{W}_{2}\right) \neq\left(W_{1}, W_{2}\right)\right\} \cup\left\{\left(\check{W}_{1}, \check{W}_{2}\right) \neq\left(W_{1}, W_{2}\right)\right\}\right) .
$$

Definition 2 ( [28]). A rate pair $\left(R_{1}, R_{2}\right)$ is said to be achievable if there exists a sequence of $\left(M_{1, n}, M_{2, n}, n\right)$ codes with $M_{1, n} \geq 2^{n R_{1}}, M_{2, n} \geq 2^{n R_{2}}$, so that $P_{e} \underset{n \rightarrow \infty}{\longrightarrow} 0$ and

$$
\begin{gathered}
\frac{1}{n} \mathbb{I}\left(W_{1}, W_{2} ; Z^{n}\right) \underset{n \rightarrow \infty}{\longrightarrow} 0 \text { for weak secrecy regime } \\
\mathbb{I}\left(W_{1}, W_{2} ; Z^{n}\right) \underset{n \rightarrow \infty}{\longrightarrow} 0 \text { for strong secrecy regime. }
\end{gathered}
$$


Definition 3. $p_{X} \approx q_{X}$ indicates $\left\|p_{X}-q_{X}\right\|_{1}<\epsilon$. For two random pmfs [27], $P_{X} \approx Q_{X}$ indicates $\mathbb{E}\left\|P_{X}-Q_{X}\right\|_{1}<\epsilon$.

\section{Achievable Rate Region Under Weak Secrecy}

We start with a lemma that fits Marton coding with indirect decoding in a MAC structure and produces an entropy bound needed in the secrecy analysis. Its basic idea can be highlighted as follows: given $X^{n}$, if we independently produce $2^{n R}$ random codevectors $Y^{n}$, we will have approximately $2^{n R-\mathbb{I}\left(X^{n} ; Y^{n}\right)}$ jointly typical pairs, i.e., the "excess" rate will determine the number of jointly typical pairs. This lemma extends the basic idea of excess rate to multiple codebooks, multiple conditioning, and furthermore, a generalization is made from a counting argument to the entropy of the index of the codebook, which is essential for the subsequent secrecy analysis.

Lemma 1. Consider random variables $\left(Q, U_{0}, V_{0}, U_{1}, V_{1}, Z\right)$ distributed according to $p_{Q} p_{U_{0}, U_{1} \mid Q} p_{V_{0}, V_{1} \mid Q} p_{Z \mid U_{0}, U_{1}, V_{0}, V_{1}}$. Draw random sequences $Q^{n}, U_{0}^{n}, V_{0}^{n}$ according to $\prod_{i=1}^{n} p_{Q}\left(q_{i}\right) \quad p_{U_{0} \mid Q}\left(u_{0, i} \mid q_{i}\right) \quad p_{V_{0} \mid Q}\left(v_{0, i} \mid q_{i}\right)$. Conditioned on $U_{0}^{n}$, draw $2^{n S}$ i.i.d. copies of $U_{1}^{n}$ according to $\prod_{i=1}^{n} p_{U_{1} \mid U_{0}}\left(u_{1, i} \mid u_{0, i}\right)$, denoted $U_{1}^{n}(\ell), \ell \in \llbracket 1,2^{n S} \rrbracket$. Similarly, conditioned on $V_{0}^{n}$, draw $2^{n T}$ i.i.d. copies of $V_{1}^{n}$ according to $\prod_{i=1}^{n} p_{V_{1} \mid V_{0}}\left(v_{1, i} \mid v_{0, i}\right)$, denoted $V_{1}^{n}(k), k \in \llbracket 1,2^{n T} \rrbracket$. Let $L \in \llbracket 1,2^{n S} \rrbracket$ and $K \in \llbracket 1,2^{n T} \rrbracket$ be random variables with arbitrary pmf. If

$$
\begin{gathered}
S>\mathbb{I}\left(U_{1} ; Z \mid Q, U_{0}, V_{0}\right)+\delta_{1}(\epsilon) \\
T>\mathbb{I}\left(V_{1} ; Z \mid Q, U_{0}, V_{0}\right)+\delta_{1}(\epsilon) \\
S+T>\mathbb{I}\left(U_{1}, V_{1} ; Z \mid Q, U_{0}, V_{0}\right)+\delta_{1}(\epsilon)
\end{gathered}
$$

for a positive $\delta_{1}(\epsilon)$ and if for an arbitrary sequence $Z^{n}$,

$$
\mathbb{P}\left(\left(Q^{n}, U_{0}^{n}, V_{0}^{n}, U_{1}^{n}(L), V_{1}^{n}(K), Z^{n}\right) \in \mathcal{T}_{\epsilon}^{(n)}\right) \underset{n \rightarrow \infty}{\longrightarrow} 1
$$

there exists a positive $\delta_{2}(\epsilon) \underset{\epsilon \rightarrow 0}{\longrightarrow} 0$, such that for $n$ sufficiently large

$$
\mathbb{H}\left(L, K \mid Q^{n}, U_{0}^{n}, V_{0}^{n}, Z^{n}, \mathcal{C}\right) \leq n\left(S+T-\mathbb{I}\left(U_{1}, V_{1} ; Z \mid Q, U_{0}, V_{0}\right)\right)+n \delta_{2}(\epsilon) .
$$

where $\mathcal{C}=\left\{U_{1}^{n}(1), \ldots, U_{1}^{n}\left(2^{n S}\right), V_{1}^{n}(1), \ldots, V_{1}^{n}\left(2^{n T}\right)\right\}$

The proof is provided in Appendix A. This result is related to, and contains, [7, Lemma 1]. In particular, [7] considers a single-input channel and explores the properties of codebooks driven by this input, while observing an output $Z$. In contrast, this paper's Lemma 1 develops a 


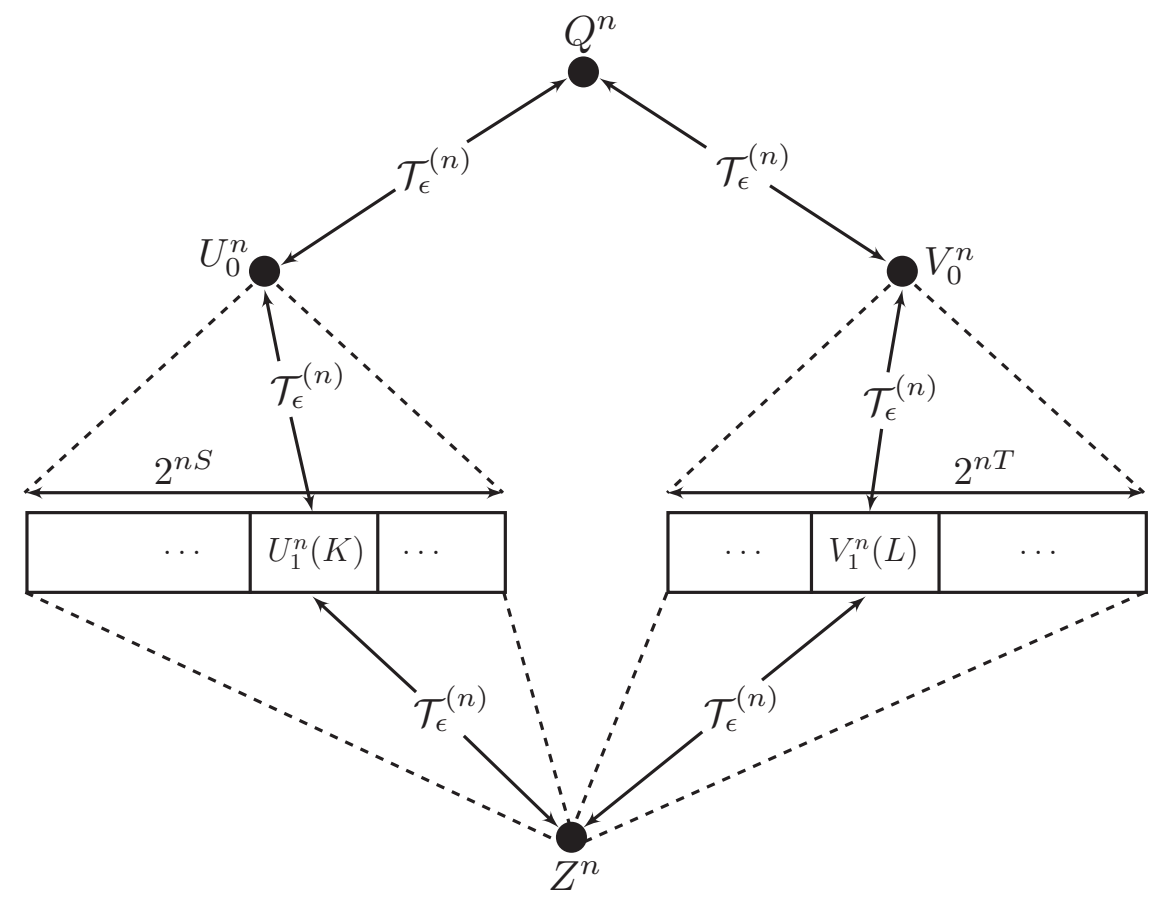

Fig. 2. Structure of Lemma 1 subject to jointly typical sequences $\left(Q^{n}, U_{0}^{n}, V_{0}^{n}, U_{1}^{n}(K), V_{1}^{n}(L), Z^{n}\right)$, finding a bound on the conditional entropy of $(K, L)$, thus implicitly bounding the number of sequence pairs that can be jointly typical with $\left(Q^{n}, Z^{n}\right)$ from codebooks with certain size.

corresponding result for a multiple-access channel with respect to $Z$, motivated by the twotransmitters present in the model of this paper. This accounts for the new features of our Lemma 1, namely three rate constraints instead of one, as well as monitoring the entropy of two index random variables instead of one. Furthermore, the present result has one additional layer of conditioning to allow for indirect decoding of multiple confidential messages in the sequel, while in [7] only one confidential message is decoded.

Remark 1. In addition to establishing the main results of this paper, Lemma 1 also has broader implications on the necessity of prefixing in multi-transmitter secrecy problems [29] and deriving the minimum amount of randomness needed to achieve secrecy. Csiszár and Körner introduced prefixing in [30] to expand the achievable rate region of the non-degraded broadcast channel with confidential messages, a technique that was subsequently used in essentially the same manner in multi-transmitter settings. Subsequently, Chia and El Gamal showed that in a single-transmitter wiretap channel, prefixing can be replaced with superposition coding [7]. Appendix B extends this concept to a multi-transmitter setting and presents an achievability technique for the multiple 
access wiretap channel that utilizes minimal randomness and matches the best known achievable rates without prefixing.

Theorem 1. An inner bound on the secrecy capacity region of the two-transmitter two-receiver channel with confidential messages is given by the set of non-negative rate pairs $\left(R_{1}, R_{2}\right)$ such that

$$
\begin{aligned}
& R_{1}<\mathbb{I}\left(U_{0}, U_{1} ; Y_{1} \mid Q, V_{0}, V_{1}\right)-\mathbb{I}\left(U_{0} ; Z \mid Q\right)-\mathbb{I}\left(U_{1} ; Z \mid U_{0}, V_{0}\right) \\
& R_{1}<\mathbb{I}\left(U_{0}, U_{2} ; Y_{2} \mid Q, V_{0}, V_{2}\right)-\mathbb{I}\left(U_{0} ; Z \mid Q\right)-\mathbb{I}\left(U_{2} ; Z \mid U_{0}, V_{0}\right) \\
& R_{1}<\mathbb{I}\left(U_{0}, U_{1}, V_{1} ; Y_{1} \mid Q, V_{0}\right)-\mathbb{I}\left(U_{0} ; Z \mid Q\right)-\mathbb{I}\left(U_{1}, V_{1} ; Z \mid U_{0}, V_{0}\right) \\
& R_{1}<\mathbb{I}\left(U_{0}, U_{2}, V_{2} ; Y_{2} \mid Q, V_{0}\right)-\mathbb{I}\left(U_{0} ; Z \mid Q\right)-\mathbb{I}\left(U_{2}, V_{2} ; Z \mid U_{0}, V_{0}\right) \\
& R_{2}<\mathbb{I}\left(V_{0}, V_{1} ; Y_{1} \mid Q, U_{0}, U_{1}\right)-\mathbb{I}\left(V_{0} ; Z \mid Q\right)-\mathbb{I}\left(V_{1} ; Z \mid U_{0}, V_{0}\right) \\
& R_{2}<\mathbb{I}\left(V_{0}, V_{2} ; Y_{2} \mid Q, U_{0}, U_{2}\right)-\mathbb{I}\left(V_{0} ; Z \mid Q\right)-\mathbb{I}\left(V_{2} ; Z \mid U_{0}, V_{0}\right) \\
& R_{2}<\mathbb{I}\left(U_{1}, V_{0}, V_{1} ; Y_{1} \mid Q, U_{0}\right)-\mathbb{I}\left(V_{0} ; Z \mid Q\right)-\mathbb{I}\left(U_{1}, V_{1} ; Z \mid U_{0}, V_{0}\right) \\
& R_{2}<\mathbb{I}\left(U_{2}, V_{0}, V_{2} ; Y_{2} \mid Q, U_{0}\right)-\mathbb{I}\left(V_{0} ; Z \mid Q\right)-\mathbb{I}\left(U_{2}, V_{2} ; Z \mid U_{0}, V_{0}\right) \\
& R_{1}+R_{2}<\mathbb{I}\left(U_{0}, U_{1}, V_{0}, V_{1} ; Y_{1} \mid Q\right)-\mathbb{I}\left(U_{0}, U_{1}, V_{0}, V_{1} ; Z \mid Q\right) \\
& R_{1}+R_{2}<\mathbb{I}\left(U_{0}, U_{2}, V_{0}, V_{2} ; Y_{2} \mid Q\right)-\mathbb{I}\left(U_{0}, U_{2}, V_{0}, V_{2} ; Z \mid Q\right) \\
& R_{1}+R_{2}<\mathbb{I}\left(U_{0}, U_{1} ; Y_{1} \mid Q, V_{0}, V_{1}\right)+\mathbb{I}\left(U_{1}, V_{0}, V_{1} ; Y_{1} \mid Q, U_{0}\right) \\
& -\mathbb{I}\left(U_{0}, U_{1}, V_{0}, V_{1} ; Z \mid Q\right)-\mathbb{I}\left(U_{1} ; Z \mid U_{0}, V_{0}\right) \\
& R_{1}+R_{2}<\mathbb{I}\left(U_{0}, U_{1} ; Y_{1} \mid Q, V_{0}, V_{1}\right)+\mathbb{I}\left(V_{0}, V_{2} ; Y_{2} \mid Q, U_{0}, U_{2}\right) \\
& -\mathbb{I}\left(U_{0}, V_{0} ; Z \mid Q\right)-\mathbb{I}\left(U_{1} ; Z \mid U_{0}, V_{0}\right)-\mathbb{I}\left(V_{2} ; Z \mid U_{0}, V_{0}\right) \\
& R_{1}+R_{2}<\mathbb{I}\left(U_{0}, U_{1} ; Y_{1} \mid Q, V_{0}, V_{1}\right)+\mathbb{I}\left(U_{2}, V_{0}, V_{2} ; Y_{2} \mid Q, U_{0}\right) \\
& -\mathbb{I}\left(U_{1} ; Z \mid U_{0}, V_{0}\right)-\mathbb{I}\left(U_{0}, U_{2}, V_{0}, V_{2} ; Z \mid Q\right) \\
& R_{1}+R_{2}<\mathbb{I}\left(V_{0}, V_{1} ; Y_{1} \mid Q, U_{0}, U_{1}\right)+\mathbb{I}\left(U_{0}, U_{1}, V_{1} ; Y_{1} \mid Q, V_{0}\right) \\
& -\mathbb{I}\left(U_{0}, U_{1}, V_{0}, V_{1} ; Z \mid Q\right)-\mathbb{I}\left(V_{1} ; Z \mid U_{0}, V_{0}\right) \\
& R_{1}+R_{2}<\mathbb{I}\left(V_{0}, V_{1} ; Y_{1} \mid Q, U_{0}, U_{1}\right)+\mathbb{I}\left(U_{0}, U_{2} ; Y_{2} \mid Q, V_{0}, V_{2}\right) \\
& -\mathbb{I}\left(U_{0}, V_{0} ; Z \mid Q\right)-\mathbb{I}\left(V_{1} ; Z \mid U_{0}, V_{0}\right)-\mathbb{I}\left(U_{2} ; Z \mid U_{0}, V_{0}\right) \\
& R_{1}+R_{2}<\mathbb{I}\left(V_{0}, V_{1} ; Y_{1} \mid Q, U_{0}, U_{1}\right)+\mathbb{I}\left(U_{0}, U_{2}, V_{2} ; Y_{2} \mid Q, V_{0}\right)
\end{aligned}
$$




$$
\begin{aligned}
& -\mathbb{I}\left(V_{1} ; Z \mid U_{0}, V_{0}\right)-\mathbb{I}\left(U_{0}, U_{2}, V_{0}, V_{2} ; Z \mid Q\right) \\
& R_{1}+R_{2}<\mathbb{I}\left(U_{0}, U_{2} ; Y_{2} \mid Q, V_{0}, V_{2}\right)+\mathbb{I}\left(U_{1}, V_{0}, V_{1} ; Y_{1} \mid Q, U_{0}\right) \\
& -\mathbb{I}\left(U_{0}, U_{1}, V_{0}, V_{1} ; Z \mid Q\right)-\mathbb{I}\left(U_{2} ; Z \mid U_{0}, V_{0}\right) \\
& R_{1}+R_{2}<\mathbb{I}\left(U_{0}, U_{2} ; Y_{2} \mid Q, V_{0}, V_{2}\right)+\mathbb{I}\left(U_{2}, V_{0}, V_{2} ; Y_{2} \mid Q, U_{0}\right) \\
& -\mathbb{I}\left(U_{0}, U_{2}, V_{0}, V_{2} ; Z \mid Q\right)-\mathbb{I}\left(U_{2} ; Z \mid U_{0}, V_{0}\right) \\
& R_{1}+R_{2}<\mathbb{I}\left(V_{0}, V_{2} ; Y_{2} \mid Q, U_{0}, U_{2}\right)+\mathbb{I}\left(U_{0}, U_{2}, V_{2} ; Y_{2} \mid Q, V_{0}\right) \\
& -\mathbb{I}\left(U_{0}, U_{2}, V_{0}, V_{2} ; Z \mid Q\right)-\mathbb{I}\left(V_{2} ; Z \mid U_{0}, V_{0}\right) \\
& R_{1}+R_{2}<\mathbb{I}\left(V_{0}, V_{2} ; Y_{2} \mid Q, U_{0}, U_{2}\right)+\mathbb{I}\left(U_{0}, U_{1}, V_{1} ; Y_{1} \mid Q, V_{0}\right) \\
& -\mathbb{I}\left(U_{0}, U_{1}, V_{0}, V_{1} ; Z \mid Q\right)-\mathbb{I}\left(V_{2} ; Z \mid U_{0}, V_{0}\right) \\
& R_{1}+R_{2}<\mathbb{I}\left(U_{0}, U_{1}, V_{1} ; Y_{1} \mid Q, V_{0}\right)+\mathbb{I}\left(U_{1}, V_{0}, V_{1} ; Y_{1} \mid Q, U_{0}\right) \\
& -\mathbb{I}\left(U_{0}, V_{0} ; Z \mid Q\right)-2 \mathbb{I}\left(U_{1}, V_{1} ; Z \mid U_{0}, V_{0}\right) \\
& R_{1}+R_{2}<\mathbb{I}\left(U_{0}, U_{1}, V_{1} ; Y_{1} \mid Q, V_{0}\right)+\mathbb{I}\left(U_{2}, V_{0}, V_{2} ; Y_{2} \mid Q, U_{0}\right) \\
& -\mathbb{I}\left(U_{0}, V_{0} ; Z \mid Q\right)-\mathbb{I}\left(U_{1}, V_{1} ; Z \mid U_{0}, V_{0}\right)-\mathbb{I}\left(U_{2}, V_{2} ; Z \mid U_{0}, V_{0}\right) \\
& R_{1}+R_{2}<\mathbb{I}\left(U_{1}, V_{0}, V_{1} ; Y_{1} \mid Q, U_{0}\right)+\mathbb{I}\left(U_{0}, U_{2}, V_{2} ; Y_{2} \mid Q, V_{0}\right) \\
& -\mathbb{I}\left(U_{0}, U_{1}, V_{0}, V_{1} ; Z \mid Q\right)-\mathbb{I}\left(U_{2}, V_{2} ; Z \mid U_{0}, V_{0}\right) \\
& R_{1}+R_{2}<\mathbb{I}\left(U_{0}, U_{2}, V_{2} ; Y_{2} \mid Q, V_{0}\right)+\mathbb{I}\left(U_{2}, V_{0}, V_{2} ; Y_{2} \mid Q, U_{0}\right) \\
& -\mathbb{I}\left(U_{0}, V_{0} ; Z \mid Q\right)-2 \mathbb{I}\left(U_{2}, V_{2} ; Z \mid U_{0}, V_{0}\right)
\end{aligned}
$$

for some

$$
\begin{aligned}
& p(q) p\left(u_{0} \mid q\right) p\left(u_{1}, u_{2} \mid u_{0}\right) p\left(v_{0} \mid q\right) p\left(v_{1}, v_{2} \mid v_{0}\right) \\
& p\left(x_{1} \mid u_{0}, u_{1}, u_{2}\right) p\left(x_{2} \mid v_{0}, v_{1}, v_{2}\right) p\left(y_{1}, y_{2}, z \mid x_{1}, x_{2}\right)
\end{aligned}
$$

such that

$$
\begin{aligned}
& \mathbb{I}\left(U_{1}, U_{2}, V_{1}, V_{2} ; Z \mid U_{0}, V_{0}\right) \leq \mathbb{I}\left(U_{1}, V_{1} ; Z \mid U_{0}, V_{0}\right) \\
& +\mathbb{I}\left(U_{2}, V_{2} ; Z \mid U_{0}, V_{0}\right)-\mathbb{I}\left(U_{1} ; U_{2} \mid U_{0}\right)-\mathbb{I}\left(V_{1} ; V_{2} \mid V_{0}\right) .
\end{aligned}
$$

The proof uses superposition coding, Wyner's wiretap coding, Marton coding, as well as indirect decoding. The details of the proof are provided in Appendix C. 


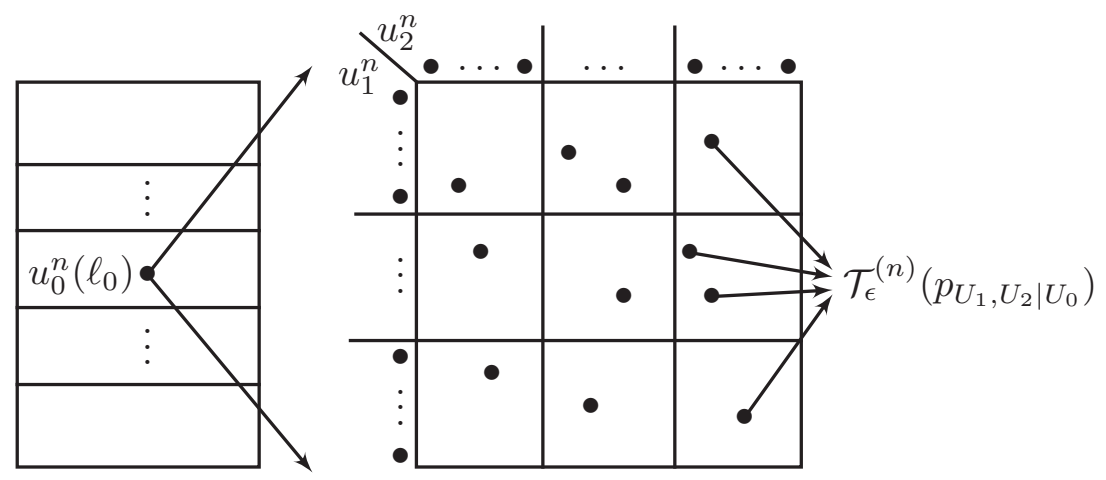

Fig. 3. Coding scheme for the first transmitter

This result covers several known earlier results:

- By setting $Z=\emptyset, U_{0}=U_{1}=U_{2}=X_{1}$, and $V_{0}=V_{1}=V_{2}=X_{2}$, the result in Theorem 1 reduces to the capacity region of compound multiple access channel discussed in [4].

- By setting $Y_{2}=\emptyset$ (or $Y_{1}=\emptyset$ ), $U_{0}=U_{1}=U_{2}=X_{1}$ and $V_{0}=V_{1}=V_{2}=X_{2}$, the result in Theorem 1 reduces to the achievable rate region of multiple access wiretap channel without common message [18]-[20].

- By setting $X_{2}=\emptyset$ ( or $X_{1}=\emptyset$ ), $U_{0}=U_{1}=U_{2}$, and $Y_{2}=\emptyset$ (or $Y_{1}=\emptyset$ ), the result in Theorem 1 reduces to the capacity region of broadcast channel with confidential message [31, Corollary 2].

- By setting $X_{2}=\emptyset$ (or $X_{1}=\emptyset$ ), the result in Theorem 1 reduces to the achievable rate region for two-receiver, one-eavesdropper wiretap channel presented in [7, Theorem 1].

Remark 2. By doing some algebraic manipulation we can show that the constraint in (5) holds only if

$$
\mathbb{I}\left(U_{1}, V_{1} ; U_{2}, V_{2} \mid U_{0}, V_{0}, Z\right)=0 .
$$

Intuitively speaking, (6) shows that the Marton coding codebooks remain independent even if the eavesdropper has access to the the cloud centers.

Corollary 1. An inner bound on the secrecy capacity region of degraded two-transmitter tworeceiver channel with confidential messages (Definition 4) is given by the set of non-negative rate pairs $\left(R_{1}, R_{2}\right)$ such that

$$
R_{1} \leq \mathbb{I}\left(U_{0} ; Y_{2} \mid V_{0}, Q\right)-\mathbb{I}\left(U_{0} ; Z \mid Q\right)
$$




$$
\begin{aligned}
R_{2} & \leq \mathbb{I}\left(V_{0} ; Y_{2} \mid U_{0}, Q\right)-\mathbb{I}\left(V_{0} ; Z \mid Q\right) \\
R_{1}+R_{2} & \leq \mathbb{I}\left(U_{0}, V_{0} ; Y_{2} \mid Q\right)-\mathbb{I}\left(U_{0} ; Z \mid Q\right)-\mathbb{I}\left(V_{0} ; Z \mid Q\right)
\end{aligned}
$$

for some

$$
p(q) p\left(u_{0} \mid q\right) p\left(v_{0} \mid q\right) p\left(x_{1} \mid u_{0}\right) p\left(x_{2} \mid v_{0}\right) \text {. }
$$

Proof. The proof follows from Theorem 1 by setting $U_{0}=U_{1}=U_{2}$ and $V_{0}=V_{1}=V_{2}$ and considering the fact that the channel is degraded.

\section{An Outer Bound For the Degraded Model}

We develop an outer bound for the degraded version of the model and provide an example in which it meets the inner bound of Theorem 1 ,

Definition 4. The degraded two-transmitter two-receiver channel with confidential messages obeys:

$$
p\left(y_{1}, y_{2}, z \mid x_{1}, x_{2}\right)=p\left(y_{1} \mid x_{1}, x_{2}\right) p\left(y_{2} \mid y_{1}\right) p\left(z \mid y_{2}\right)
$$

Theorem 2. The secrecy capacity region for the degraded two-transmitter two-receiver channel with confidential messages is included in the set of rate pairs $\left(R_{1}, R_{2}\right)$ satisfying

$$
\begin{gathered}
R_{1} \leq \mathbb{I}\left(U_{0} ; Y_{2} \mid Q\right)-\mathbb{I}\left(U_{0} ; Z \mid Q\right), \\
R_{2} \leq \mathbb{I}\left(V_{0} ; Y_{2} \mid Q\right)-\mathbb{I}\left(V_{0} ; Z \mid Q\right), \\
R_{1}+R_{2} \leq \mathbb{I}\left(U_{0}, V_{0} ; Y_{2} \mid Q\right)-\mathbb{I}\left(U_{0}, V_{0} ; Z \mid Q\right),
\end{gathered}
$$

for some joint distribution

$$
p(q) p\left(u_{0}, v_{0} \mid q\right) p\left(x_{1} \mid u_{0}\right) p\left(x_{2} \mid v_{0}\right)
$$

Proof. The proof is provided by the authors in [13, Section IV] and is omitted here for brevity.

Example (Degraded Switch Model): We consider an example of the two-transmitter tworeceiver channel where the first legitimate receiver has access to the noisy version of each of the two transmitted values in a time-sharing (switched) manner, without interference from the other transmitter (Fig. 4). The second legitimate receiver has access to a noisy version of the first receiver, and the eavesdropper has access to a noisy version of the second receiver. The 


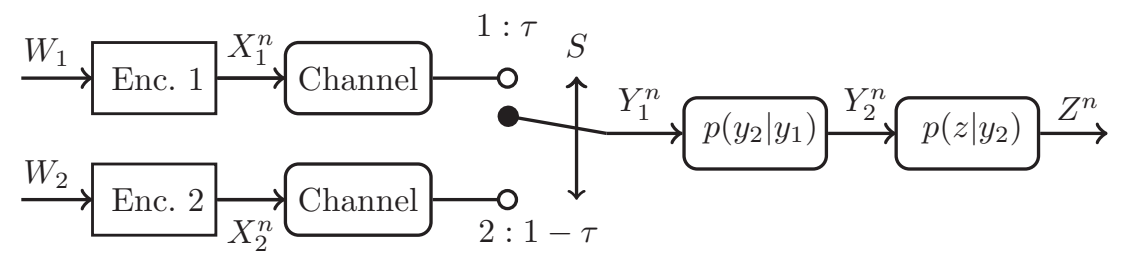

Fig. 4. Degraded switch model

switch channel state information is made available to all terminals. In this model the channel outputs are as follows:

$$
\begin{aligned}
& y_{1}^{\prime}=\left(y_{1}, s\right), \\
& y_{2}^{\prime}=\left(y_{2}, s\right), \\
& z^{\prime}=(z, s) .
\end{aligned}
$$

This model consists of a channel with states that are causally available at both the encoders and decoders.

The statistics of the channel, conditioned on the switch state, are expressed as follows:

$$
p\left(y_{1}^{\prime}, y_{2}^{\prime}, z \mid x_{1},, x_{2}, s\right)=p\left(y_{1} \mid x_{1}, x_{2}, s\right) p\left(y_{2} \mid y_{1}, s\right) p\left(z \mid y_{2}, s\right)
$$

The switch model describes, e.g., frequency hopping over two frequencies [26]. The state (switch) is a binary random variable that chooses between listening to the Transmitter 1, with probability $\tau$, and listening to the Transmitter 2 , with probability $1-\tau$, independently at each time slot. We further assume the state is i.i.d. across time.

$$
p\left(y_{1} \mid x_{1}, x_{2}, s\right)=p\left(y_{1} \mid x_{1}\right) \mathbb{1}\{s=1\}+p\left(y_{1} \mid x_{2}\right) \mathbb{1}\{s=2\}
$$

where $\mathbb{1}$ is the indicator function.

Theorem 3. The secrecy capacity region for the degraded switch two-transmitter two-receiver channel with confidential messages, is given by the set of rate pairs $\left(R_{1}, R_{2}\right)$ satisfying

$$
\begin{gathered}
R_{1} \leq \mathbb{I}\left(U_{0} ; Y_{2}^{\prime} \mid V_{0}, Q\right)-\mathbb{I}\left(U_{0} ; Z^{\prime} \mid Q\right), \\
R_{2} \leq \mathbb{I}\left(V_{0} ; Y_{2}^{\prime} \mid U_{0}, Q\right)-\mathbb{I}\left(V_{0} ; Z^{\prime} \mid Q\right), \\
R_{1}+R_{2} \leq \mathbb{I}\left(U_{0}, V_{0} ; Y_{2}^{\prime} \mid Q\right)-\mathbb{I}\left(U_{0}, V_{0} ; Z^{\prime} \mid Q\right),
\end{gathered}
$$


for some joint distribution

$$
p(q) p\left(u_{0} \mid q\right) p\left(v_{0} \mid q\right) p\left(x_{1} \mid u_{0}\right) p\left(x_{2} \mid v_{0}\right) \text {. }
$$

Proof. The proof is available in [13, Section IV].

\section{A General Outer Bound}

We now develop a general outer bound for the model of Fig. 1 and provide an example in which it meets the inner bound of Theorem 1 .

Theorem 4. The secrecy capacity region for the two-transmitter two-receiver channel with confidential messages is included in the set of rate pairs $\left(R_{1}, R_{2}\right)$ satisfying

$$
\begin{gathered}
R_{1} \leq \mathbb{I}\left(U_{0} ; Y_{1}, Y_{2} \mid Q\right)-\mathbb{I}\left(U_{0} ; Z \mid Q\right), \\
R_{2} \leq \mathbb{I}\left(V_{0} ; Y_{1}, Y_{2} \mid Q\right)-\mathbb{I}\left(V_{0} ; Z \mid Q\right), \\
R_{1}+R_{2} \leq \mathbb{I}\left(U_{0}, V_{0} ; Y_{1}, Y_{2} \mid Q\right)-\mathbb{I}\left(U_{0}, V_{0} ; Z \mid Q\right),
\end{gathered}
$$

for some joint distribution

$$
p(q) p\left(u_{0}, v_{0} \mid q\right) p\left(x_{1} \mid u_{0}\right) p\left(x_{2} \mid v_{0}\right)
$$

Proof. The proof is available in [13, Section V].

Example (Noiseless Switch Model): This example is motivated by two transmitters operating on different spectral bands, while the receiving terminals may receive adaptively on one band at a time [26]. The eavesdropper in our example has access to one noiseless interference-free transmitted value at a time. Here, it is assumed that both legitimate receivers operate according to a common random switch $s_{1}$ that is connected to Transmitter 1 with probability $\tau_{1}$ and to Transmitter 2 with probability $1-\tau_{1}$, and the eavesdropper operates according to another random switch $s_{2}$ that is connected to Transmitter 1 with probability $\tau_{2}$ and to Transmitter 2 with probability $1-\tau_{2}$. Aside from the switches, the channel is noiseless. Both receivers and the eavesdropper have access to their own switch state information. Therefore the channel outputs are considered

$$
\begin{aligned}
& y_{1}^{\prime}=\left(y_{1}, s_{1}\right), \\
& y_{2}^{\prime}=\left(y_{2}, s_{1}\right),
\end{aligned}
$$




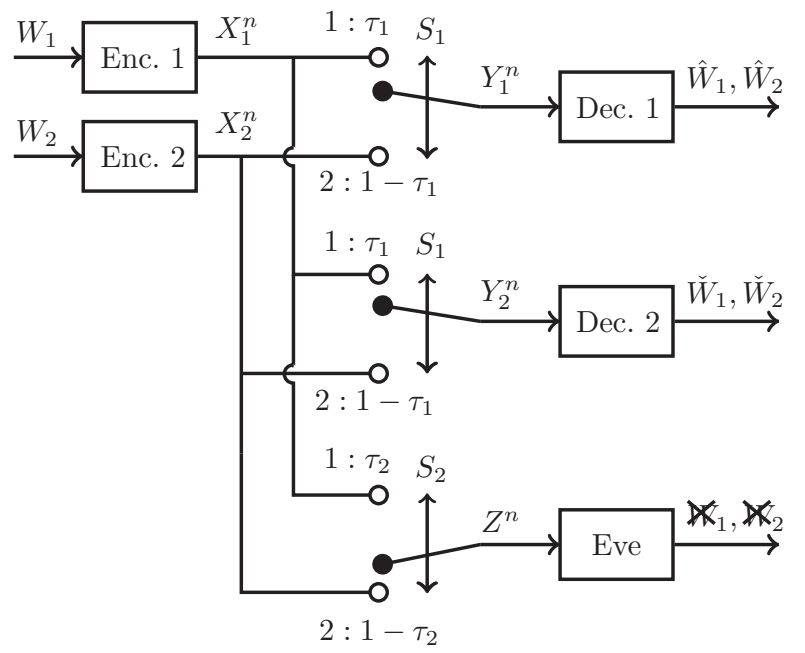

Fig. 5. Noiseless switch model

$$
z^{\prime}=\left(z, s_{2}\right)
$$

Since $y_{1}=y_{2}$, we also have $y_{1}^{\prime}=y_{2}^{\prime}$.

Theorem 5. The secrecy capacity region for the noiseless switch two-transmitter two-receiver channel with confidential messages is given by the set of rate pairs $\left(R_{1}, R_{2}\right)$ satisfying

$$
\begin{aligned}
& R_{1} \leq\left(\tau_{1}-\tau_{2}\right)^{+} \mathbb{H}\left(X_{1}\right), \\
& R_{2} \leq\left(\tau_{2}-\tau_{1}\right)^{+} \mathbb{H}\left(X_{2}\right),
\end{aligned}
$$

where $(x)^{+}=\max \{0, x\}$.

Proof. The proof is available in [13, Section V].

The capacity region in Theorem 5 shows that transmitters can securely communicate to receivers as long as $\tau_{1} \neq \tau_{2}$.

\section{Strong Secrecy}

Theorem 6. An inner bound on the secrecy capacity region of the two-transmitter two-receiver channel with confidential messages is given by the set of non-negative rate pairs $\left(R_{1}, R_{2}\right)$ such that

$R_{1}<\mathbb{I}\left(U_{0}, U_{1} ; Y_{1} \mid Q, V_{0}, V_{1}\right)-\mathbb{I}\left(U_{0} ; Z \mid Q\right)-\mathbb{I}\left(U_{1} ; Z \mid U_{0}, V_{0}\right)$ 


$$
\begin{aligned}
& R_{1}<\mathbb{I}\left(U_{0}, U_{2} ; Y_{2} \mid Q, V_{0}, V_{2}\right)-\mathbb{I}\left(U_{0} ; Z \mid Q\right)-\mathbb{I}\left(U_{2} ; Z \mid U_{0}, V_{0}\right) \\
& R_{1}<\mathbb{I}\left(U_{0}, U_{1}, V_{1} ; Y_{1} \mid Q, V_{0}\right)-\mathbb{I}\left(U_{0} ; Z \mid Q\right)-\mathbb{I}\left(U_{1}, V_{1} ; Z \mid U_{0}, V_{0}\right) \\
& R_{1}<\mathbb{I}\left(U_{0}, U_{2}, V_{2} ; Y_{2} \mid Q, V_{0}\right)-\mathbb{I}\left(U_{0} ; Z \mid Q\right)-\mathbb{I}\left(U_{2}, V_{2} ; Z \mid U_{0}, V_{0}\right) \\
& 2 R_{1}<\mathbb{I}\left(U_{0}, U_{1} ; Y_{1} \mid Q, V_{0}, V_{1}\right)+\mathbb{I}\left(U_{0}, U_{2} ; Y_{2} \mid Q, V_{0}, V_{2}\right) \\
& -2 \mathbb{I}\left(U_{0} ; Z \mid Q\right)-\mathbb{I}\left(U_{1}, U_{2} ; Z \mid U_{0}, V_{0}\right)-\mathbb{I}\left(U_{1} ; U_{2} \mid U_{0}\right) \\
& 2 R_{1}<\mathbb{I}\left(U_{0}, U_{1} ; Y_{1} \mid Q, V_{0}, V_{1}\right)+\mathbb{I}\left(U_{0}, U_{2}, V_{2} ; Y_{2} \mid Q, V_{0}\right) \\
& -2 \mathbb{I}\left(U_{0} ; Z \mid Q\right)-\mathbb{I}\left(U_{1}, U_{2}, V_{2} ; Z \mid U_{0}, V_{0}\right)-\mathbb{I}\left(U_{1} ; U_{2} \mid U_{0}\right) \\
& 2 R_{1}<\mathbb{I}\left(U_{0}, U_{2} ; Y_{2} \mid Q, V_{0}, V_{2}\right)+\mathbb{I}\left(U_{0}, U_{1}, V_{1} ; Y_{1} \mid Q, V_{0}\right) \\
& -2 \mathbb{I}\left(U_{0} ; Z \mid Q\right)-\mathbb{I}\left(U_{1}, U_{2}, V_{1} ; Z \mid U_{0}, V_{0}\right)-\mathbb{I}\left(U_{1} ; U_{2} \mid U_{0}\right) \\
& 2 R_{1}<\mathbb{I}\left(U_{0}, U_{1}, V_{1} ; Y_{1} \mid Q, U_{0}\right)+\mathbb{I}\left(U_{0}, U_{2}, V_{2} ; Y_{2} \mid Q, U_{0}\right) \\
& -2 \mathbb{I}\left(U_{0} ; Z \mid Q\right)-\mathbb{I}\left(U_{1}, U_{2}, V_{1}, V_{2} ; Z \mid U_{0}, V_{0}\right)-\mathbb{I}\left(U_{1}, V_{1} ; U_{2}, V_{2} \mid U_{0}, V_{0}\right) \\
& R_{2}<\mathbb{I}\left(V_{0}, V_{1} ; Y_{1} \mid Q, U_{0}, U_{1}\right)-\mathbb{I}\left(V_{0} ; Z \mid Q\right)-\mathbb{I}\left(V_{1} ; Z \mid U_{0}, V_{0}\right) \\
& R_{2}<\mathbb{I}\left(V_{0}, V_{2} ; Y_{2} \mid Q, U_{0}, U_{2}\right)-\mathbb{I}\left(V_{0} ; Z \mid Q\right)-\mathbb{I}\left(V_{2} ; Z \mid U_{0}, V_{0}\right) \\
& R_{2}<\mathbb{I}\left(U_{1}, V_{0}, V_{1} ; Y_{1} \mid Q, U_{0}\right)-\mathbb{I}\left(V_{0} ; Z \mid Q\right)-\mathbb{I}\left(U_{1}, V_{1} ; Z \mid U_{0}, V_{0}\right) \\
& R_{2}<\mathbb{I}\left(U_{2}, V_{0}, V_{2} ; Y_{2} \mid Q, U_{0}\right)-\mathbb{I}\left(V_{0} ; Z \mid Q\right)-\mathbb{I}\left(U_{2}, V_{2} ; Z \mid U_{0}, V_{0}\right) \\
& 2 R_{2}<\mathbb{I}\left(V_{0}, V_{1} ; Y_{1} \mid Q, U_{0}, U_{1}\right)+\mathbb{I}\left(V_{0}, V_{2} ; Y_{2} \mid Q, U_{0}, U_{2}\right) \\
& -2 \mathbb{I}\left(V_{0} ; Z \mid Q\right)-\mathbb{I}\left(V_{1}, V_{2} ; Z \mid U_{0}, V_{0}\right)-\mathbb{I}\left(V_{1} ; V_{2} \mid V_{0}\right) \\
& 2 R_{2}<\mathbb{I}\left(V_{0}, V_{1} ; Y_{1} \mid Q, U_{0}, U_{1}\right)+\mathbb{I}\left(U_{2}, V_{0}, V_{2} ; Y_{2} \mid Q, U_{0}\right) \\
& -2 \mathbb{I}\left(V_{0} ; Z \mid Q\right)-\mathbb{I}\left(U_{2}, V_{1}, V_{2} ; Z \mid U_{0}, V_{0}\right)-\mathbb{I}\left(V_{1} ; V_{2} \mid V_{0}\right) \\
& 2 R_{2}<\mathbb{I}\left(V_{0}, V_{2} ; Y_{2} \mid Q, U_{0}, U_{2}\right)+\mathbb{I}\left(U_{1}, V_{0}, V_{1} ; Y_{1} \mid Q, U_{0}\right) \\
& -2 \mathbb{I}\left(V_{0} ; Z \mid Q\right)-\mathbb{I}\left(U_{1}, V_{1}, V_{2} ; Z \mid U_{0}, V_{0}\right)-\mathbb{I}\left(V_{1} ; V_{2} \mid V_{0}\right) \\
& 2 R_{2}<\mathbb{I}\left(U_{1}, V_{0}, V_{1} ; Y_{1} \mid Q, U_{0}\right)+\mathbb{I}\left(U_{2}, V_{0}, V_{2} ; Y_{2} \mid Q, U_{0}\right) \\
& -2 \mathbb{I}\left(V_{0} ; Z \mid Q\right)-\mathbb{I}\left(U_{1}, U_{2}, V_{1}, V_{2} ; Z \mid U_{0}, V_{0}\right)-\mathbb{I}\left(U_{1}, V_{1} ; U_{2}, V_{2} \mid U_{0}, V_{0}\right) \\
& R_{1}+R_{2}<\mathbb{I}\left(U_{0}, U_{1} ; Y_{1} \mid Q, V_{0}, V_{1}\right)+\mathbb{I}\left(V_{0}, V_{2} ; Y_{2} \mid Q, U_{0}, U_{2}\right) \\
& -\mathbb{I}\left(U_{0}, V_{0} ; Z \mid Q\right)-\mathbb{I}\left(U_{1}, V_{2} ; Z \mid U_{0}, V_{0}\right)
\end{aligned}
$$




$$
\begin{aligned}
& R_{1}+R_{2}<\mathbb{I}\left(U_{0}, U_{1} ; Y_{1} \mid Q, V_{0}, V_{1}\right)+\mathbb{I}\left(U_{1}, V_{0}, V_{1} ; Y_{1} \mid Q, U_{0}\right) \\
& -\mathbb{I}\left(U_{0}, V_{0} ; Z \mid Q\right)-\mathbb{I}\left(U_{1} ; Z \mid U_{0}, V_{0}\right)-\mathbb{I}\left(U_{1}, V_{1} ; Z \mid U_{0}, V_{0}\right) \\
& R_{1}+R_{2}<\mathbb{I}\left(U_{0}, U_{1} ; Y_{1} \mid Q, V_{0}, V_{1}\right)+\mathbb{I}\left(U_{2}, V_{0}, V_{2} ; Y_{2} \mid Q, U_{0}\right) \\
& -\mathbb{I}\left(U_{0}, V_{0} ; Z \mid Q\right)-\mathbb{I}\left(U_{1}, U_{2}, V_{2} ; Z \mid U_{0}, V_{0}\right)-\mathbb{I}\left(U_{1} ; U_{2} \mid U_{0}\right) \\
& R_{1}+R_{2}<\mathbb{I}\left(U_{0}, U_{2} ; Y_{2} \mid Q, V_{0}, V_{2}\right)+\mathbb{I}\left(V_{0}, V_{1} ; Y_{1} \mid Q, U_{0}, U_{1}\right) \\
& -\mathbb{I}\left(U_{0}, V_{0} ; Z \mid Q\right)-\mathbb{I}\left(U_{2}, V_{1} ; Z \mid U_{0}, V_{0}\right) \\
& R_{1}+R_{2}<\mathbb{I}\left(U_{0}, U_{2} ; Y_{2} \mid Q, V_{0}, V_{2}\right)+\mathbb{I}\left(U_{1}, V_{0}, V_{1} ; Y_{1} \mid Q, U_{0}\right) \\
& -\mathbb{I}\left(U_{0}, V_{0} ; Z \mid Q\right)-\mathbb{I}\left(U_{1}, U_{2}, V_{1} ; Z \mid U_{0}, V_{0}\right)-\mathbb{I}\left(U_{1} ; U_{2} \mid U_{0}\right) \\
& R_{1}+R_{2}<\mathbb{I}\left(U_{0}, U_{2} ; Y_{2} \mid Q, V_{0}, V_{2}\right)+\mathbb{I}\left(U_{2}, V_{0}, V_{2} ; Y_{2} \mid Q, U_{0}\right) \\
& -\mathbb{I}\left(U_{0}, V_{0} ; Z \mid Q\right)-\mathbb{I}\left(U_{2} ; Z \mid U_{0}, V_{0}\right)-\mathbb{I}\left(U_{2}, V_{2} ; Z \mid U_{0}, V_{0}\right) \\
& R_{1}+R_{2}<\mathbb{I}\left(V_{0}, V_{1} ; Y_{1} \mid Q, U_{0}, U_{1}\right)+\mathbb{I}\left(U_{0}, U_{1}, V_{1} ; Y_{1} \mid Q, V_{0}\right) \\
& -\mathbb{I}\left(U_{0}, V_{0} ; Z \mid Q\right)-\mathbb{I}\left(V_{1} ; Z \mid U_{0}, V_{0}\right)-\mathbb{I}\left(U_{1}, V_{1} ; Z \mid U_{0}, V_{0}\right) \\
& R_{1}+R_{2}<\mathbb{I}\left(V_{0}, V_{1} ; Y_{1} \mid Q, U_{0}, U_{1}\right)+\mathbb{I}\left(U_{0}, U_{2}, V_{2} ; Y_{2} \mid Q, V_{0}\right) \\
& -\mathbb{I}\left(U_{0}, V_{0} ; Z \mid Q\right)-\mathbb{I}\left(U_{2}, V_{1}, V_{2} ; Z \mid U_{0}, V_{0}\right)-\mathbb{I}\left(V_{1} ; V_{2} \mid V_{0}\right) \\
& R_{1}+R_{2}<\mathbb{I}\left(V_{0}, V_{2} ; Y_{2} \mid Q, U_{0}, U_{2}\right)+\mathbb{I}\left(U_{0}, U_{1}, V_{1} ; Y_{1} \mid Q, V_{0}\right) \\
& -\mathbb{I}\left(U_{0}, V_{0} ; Z \mid Q\right)-\mathbb{I}\left(U_{1}, V_{1}, V_{2} ; Z \mid U_{0}, V_{0}\right)-\mathbb{I}\left(V_{1} ; V_{2} \mid V_{0}\right) \\
& R_{1}+R_{2}<\mathbb{I}\left(V_{0}, V_{2} ; Y_{2} \mid Q, U_{0}, U_{2}\right)+\mathbb{I}\left(U_{0}, U_{2}, V_{2} ; Y_{2} \mid Q, V_{0}\right) \\
& -\mathbb{I}\left(U_{0}, V_{0} ; Z \mid Q\right)-\mathbb{I}\left(V_{2} ; Z \mid U_{0}, V_{0}\right)-\mathbb{I}\left(U_{2}, V_{2} ; Z \mid U_{0}, V_{0}\right) \\
& R_{1}+R_{2}<\mathbb{I}\left(U_{0}, U_{1}, V_{1} ; Y_{1} \mid Q, V_{0}\right)+\mathbb{I}\left(U_{1}, V_{0}, V_{1} ; Y_{1} \mid Q, U_{0}\right) \\
& -\mathbb{I}\left(U_{0}, V_{0} ; Z \mid Q\right)-2 \mathbb{I}\left(U_{1}, V_{1} ; Z \mid U_{0}, V_{0}\right) \\
& R_{1}+R_{2}<\mathbb{I}\left(U_{0}, U_{1}, V_{1} ; Y_{1} \mid Q, V_{0}\right)+\mathbb{I}\left(U_{2}, V_{0}, V_{2} ; Y_{2} \mid Q, U_{0}\right)-\mathbb{I}\left(U_{0}, V_{0} ; Z \mid Q\right) \\
& -\mathbb{I}\left(U_{1}, U_{2}, V_{1}, V_{2} ; Z \mid U_{0}, V_{0}\right)-\mathbb{I}\left(U_{1}, V_{1} ; U_{2}, V_{2} \mid U_{0}, V_{0}\right) \\
& R_{1}+R_{2}<\mathbb{I}\left(U_{0}, U_{2}, V_{2} ; Y_{2} \mid Q, V_{0}\right)+\mathbb{I}\left(U_{1}, V_{0}, V_{1} ; Y_{1} \mid Q, U_{0}\right)-\mathbb{I}\left(U_{0}, V_{0} ; Z \mid Q\right) \\
& -\mathbb{I}\left(U_{1}, U_{2}, V_{1}, V_{2} ; Z \mid U_{0}, V_{0}\right)-\mathbb{I}\left(U_{1}, V_{1} ; U_{2}, V_{2} \mid U_{0}, V_{0}\right) \\
& R_{1}+R_{2}<\mathbb{I}\left(U_{0}, U_{2}, V_{2} ; Y_{2} \mid Q, V_{0}\right)+\mathbb{I}\left(U_{2}, V_{0}, V_{2} ; Y_{2} \mid Q, U_{0}\right)
\end{aligned}
$$




$$
\begin{aligned}
& -\mathbb{I}\left(U_{0}, V_{0} ; Z \mid Q\right)-2 \mathbb{I}\left(U_{2}, V_{2} ; Z \mid U_{0}, V_{0}\right) \\
& R_{1}+R_{2}<\mathbb{I}\left(U_{0}, U_{2}, V_{0}, V_{2} ; Y_{2} \mid Q\right)-\mathbb{I}\left(U_{0}, U_{2}, V_{0}, V_{2} ; Z \mid Q\right) \\
& R_{1}+R_{2}<\mathbb{I}\left(U_{0}, U_{1}, V_{0}, V_{1} ; Y_{1} \mid Q\right)-\mathbb{I}\left(U_{0}, U_{1}, V_{0}, V_{1} ; Z \mid Q\right) \\
& 2 R_{1}+R_{2}<\mathbb{I}\left(U_{0}, U_{1} ; Y_{1} \mid Q, V_{0}, V_{1}\right)+\mathbb{I}\left(U_{0}, U_{2}, V_{0}, V_{2} ; Y_{2} \mid Q\right) \\
& -\mathbb{I}\left(U_{0} ; Z \mid Q\right)-\mathbb{I}\left(U_{0}, V_{0} ; Z \mid Q\right)-\mathbb{I}\left(U_{1}, U_{2}, V_{2} ; Z \mid U_{0}, V_{0}\right)-\mathbb{I}\left(U_{1} ; U_{2} \mid U_{0}\right) \\
& 2 R_{1}+R_{2}<\mathbb{I}\left(U_{0}, U_{2} ; Y_{2} \mid Q, V_{0}, V_{2}\right)+\mathbb{I}\left(U_{0}, U_{1}, V_{0}, V_{1} ; Y_{1} \mid Q\right) \\
& -\mathbb{I}\left(U_{0} ; Z \mid Q\right)-\mathbb{I}\left(U_{0}, V_{0} ; Z \mid Q\right)-\mathbb{I}\left(U_{1}, U_{2}, V_{1} ; Z \mid U_{0}, V_{0}\right)-\mathbb{I}\left(U_{1} ; U_{2} \mid U_{0}\right) \\
& 2 R_{1}+R_{2}<\mathbb{I}\left(U_{0}, U_{1}, V_{1} ; Y_{1} \mid Q, V_{0}\right)+\mathbb{I}\left(U_{0}, U_{2}, V_{0}, V_{2} ; Y_{2} \mid Q\right) \\
& -\mathbb{I}\left(U_{0} ; Z \mid Q\right)-\mathbb{I}\left(U_{0}, V_{0} ; Z \mid Q\right)-\mathbb{I}\left(U_{1}, U_{2}, V_{1}, V_{2} ; Z \mid U_{0}, V_{0}\right)-\mathbb{I}\left(U_{1}, V_{1} ; U_{2}, V_{2} \mid U_{0}, V_{0}\right) \\
& 2 R_{1}+R_{2}<\mathbb{I}\left(U_{0}, U_{2}, V_{2} ; Y_{2} \mid Q, V_{0}\right)+\mathbb{I}\left(U_{0}, U_{1}, V_{0}, V_{1} ; Y_{1} \mid Q\right) \\
& -\mathbb{I}\left(U_{0} ; Z \mid Q\right)-\mathbb{I}\left(U_{0}, V_{0} ; Z \mid Q\right)-\mathbb{I}\left(U_{1}, U_{2}, V_{1}, V_{2} ; Z \mid U_{0}, V_{0}\right)-\mathbb{I}\left(U_{1}, V_{1} ; U_{2}, V_{2} \mid U_{0}, V_{0}\right) \\
& R_{1}+2 R_{2}<\mathbb{I}\left(V_{0}, V_{1} ; Y_{1} \mid Q, U_{0}, U_{1}\right)+\mathbb{I}\left(U_{0}, U_{2}, V_{0}, V_{2} ; Y_{2} \mid Q\right) \\
& -\mathbb{I}\left(V_{0} ; Z \mid Q\right)-\mathbb{I}\left(U_{0}, V_{0} ; Z \mid Q\right)-\mathbb{I}\left(U_{2}, V_{1}, V_{2} ; Z \mid U_{0}, V_{0}\right)-\mathbb{I}\left(V_{1} ; V_{2} \mid V_{0}\right) \\
& R_{1}+2 R_{2}<\mathbb{I}\left(V_{0}, V_{2} ; Y_{2} \mid Q, U_{0}, U_{2}\right)+\mathbb{I}\left(U_{0}, U_{1}, V_{0}, V_{1} ; Y_{1} \mid Q\right) \\
& -\mathbb{I}\left(V_{0} ; Z \mid Q\right)-\mathbb{I}\left(U_{0}, V_{0} ; Z \mid Q\right)-\mathbb{I}\left(U_{1}, V_{1}, V_{2} ; Z \mid U_{0}, V_{0}\right)-\mathbb{I}\left(V_{1} ; V_{2} \mid V_{0}\right) \\
& R_{1}+2 R_{2}<\mathbb{I}\left(U_{0}, U_{1}, V_{0}, V_{1} ; Y_{1} \mid Q\right)+\mathbb{I}\left(U_{2}, V_{0}, V_{2} ; Y_{2} \mid Q, U_{0}\right) \\
& -\mathbb{I}\left(V_{0} ; Z \mid Q\right)-\mathbb{I}\left(U_{0}, V_{0} ; Z \mid Q\right)-\mathbb{I}\left(U_{1}, U_{2}, V_{1}, V_{2} ; Z \mid U_{0}, V_{0}\right)-\mathbb{I}\left(U_{1}, V_{1} ; U_{2}, V_{2} \mid U_{0}, V_{0}\right) \\
& R_{1}+2 R_{2}<\mathbb{I}\left(U_{1}, V_{0}, V_{1} ; Y_{1} \mid Q, U_{0}\right)+\mathbb{I}\left(U_{0}, U_{2}, V_{0}, V_{2} ; Y_{2} \mid Q\right) \\
& -\mathbb{I}\left(V_{0} ; Z \mid Q\right)-\mathbb{I}\left(U_{0}, V_{0} ; Z \mid Q\right)-\mathbb{I}\left(U_{1}, U_{2}, V_{1}, V_{2} ; Z \mid U_{0}, V_{0}\right)-\mathbb{I}\left(U_{1}, V_{1} ; U_{2}, V_{2} \mid U_{0}, V_{0}\right) \\
& 2 R_{1}+2 R_{2}<2 \mathbb{I}\left(U_{0}, U_{1} ; Y_{1} \mid Q, V_{0}, V_{1}\right)+\mathbb{I}\left(V_{0}, V_{2} ; Y_{2} \mid Q, U_{0}, U_{2}\right)+\mathbb{I}\left(U_{1}, V_{0}, V_{1} ; Y_{1} \mid Q, U_{0}\right) \\
& -2 \mathbb{I}\left(U_{0}, V_{0} ; Z \mid Q\right)-2 \mathbb{I}\left(U_{1} ; Z \mid U_{0}, V_{0}\right)-\mathbb{I}\left(U_{1}, V_{1}, V_{2} ; Z \mid U_{0}, V_{0}\right)-\mathbb{I}\left(V_{1} ; V_{2} \mid V_{0}\right) \\
& 2 R_{1}+2 R_{2}<2 \mathbb{I}\left(U_{0}, U_{1} ; Y_{1} \mid Q, V_{0}, V_{1}\right)+\mathbb{I}\left(U_{1}, V_{0}, V_{1} ; Y_{1} \mid Q, U_{0}\right)+\mathbb{I}\left(U_{2}, V_{0}, V_{2} ; Y_{2} \mid Q, U_{0}\right) \\
& -2 \mathbb{I}\left(U_{0}, V_{0} ; Z \mid Q\right)-2 \mathbb{I}\left(U_{1} ; Z \mid U_{0}, V_{0}\right)-\mathbb{I}\left(U_{1}, U_{2}, V_{1}, V_{2} ; Z \mid U_{0}, V_{0}\right)-\mathbb{I}\left(U_{1}, V_{1} ; U_{2}, V_{2} \mid U_{0}, V_{0}\right) \\
& 2 R_{1}+2 R_{2}<\mathbb{I}\left(U_{0}, U_{1} ; Y_{1} \mid Q, V_{0}, V_{1}\right)+\mathbb{I}\left(U_{0}, U_{2} ; Y_{2} \mid Q, V_{0}, V_{2}\right) \\
& +\mathbb{I}\left(U_{1}, V_{0}, V_{1} ; Y_{1} \mid Q, U_{0}\right)+\mathbb{I}\left(U_{2}, V_{0}, V_{2} ; Y_{2} \mid Q, U_{0}\right)-2 \mathbb{I}\left(U_{0}, V_{0} ; Z \mid Q\right)-\mathbb{I}\left(U_{1}, U_{2} ; Z \mid U_{0}, V_{0}\right)
\end{aligned}
$$


$-\mathbb{I}\left(U_{1}, U_{2}, V_{1}, V_{2} ; Z \mid U_{0}, V_{0}\right)-\mathbb{I}\left(U_{1} ; U_{2} \mid U_{0}\right)-\mathbb{I}\left(U_{1}, V_{1} ; U_{2}, V_{2} \mid U_{0}, V_{0}\right)$

$$
2 R_{1}+2 R_{2}<\mathbb{I}\left(U_{0}, U_{1} ; Y_{1} \mid Q, V_{0}, V_{1}\right)+2 \mathbb{I}\left(V_{0}, V_{2} ; Y_{2} \mid Q, U_{0}, U_{2}\right)+\mathbb{I}\left(U_{0}, U_{2}, V_{2} ; Y_{2} \mid Q, V_{0}\right)
$$

$-2 \mathbb{I}\left(U_{0}, V_{0} ; Z \mid Q\right)-2 \mathbb{I}\left(V_{2} ; Z \mid U_{0}, V_{0}\right)-\mathbb{I}\left(U_{1}, U_{2}, V_{2} ; Z \mid U_{0}, V_{0}\right)-\mathbb{I}\left(U_{1} ; U_{2} \mid U_{0}\right)$

$$
\begin{gathered}
2 R_{1}+2 R_{2}<\mathbb{I}\left(U_{0}, U_{1} ; Y_{1} \mid Q, V_{0}, V_{1}\right)+\mathbb{I}\left(V_{0}, V_{2} ; Y_{2} \mid Q, U_{0}, U_{2}\right)+\mathbb{I}\left(U_{0}, U_{1}, V_{0}, V_{1} ; Y_{1} \mid Q\right) \\
-2 \mathbb{I}\left(U_{0}, V_{0} ; Z \mid Q\right)-\mathbb{I}\left(U_{1} ; Z \mid U_{0}, V_{0}\right)-\mathbb{I}\left(U_{1}, V_{1}, V_{2} ; Z \mid U_{0}, V_{0}\right)-\mathbb{I}\left(V_{1} ; V_{2} \mid V_{0}\right)
\end{gathered}
$$$$
2 R_{1}+2 R_{2}<\mathbb{I}\left(U_{0}, U_{1} ; Y_{1} \mid Q, V_{0}, V_{1}\right)+\mathbb{I}\left(V_{0}, V_{2} ; Y_{2} \mid Q, U_{0}, U_{2}\right)+\mathbb{I}\left(U_{0}, U_{2}, V_{0}, V_{2} ; Y_{2} \mid Q\right)
$$$$
-2 \mathbb{I}\left(U_{0}, V_{0} ; Z \mid Q\right)-\mathbb{I}\left(V_{2} ; Z \mid U_{0}, V_{0}\right)-\mathbb{I}\left(U_{1}, U_{2}, V_{2} ; Z \mid U_{0}, V_{0}\right)-\mathbb{I}\left(U_{1} ; U_{2} \mid U_{0}\right)
$$

$$
2 R_{1}+2 R_{2}<\mathbb{I}\left(U_{0}, U_{1} ; Y_{1} \mid Q, V_{0}, V_{1}\right)+\mathbb{I}\left(U_{1}, V_{0}, V_{1} ; Y_{1} \mid Q, U_{0}\right)+\mathbb{I}\left(U_{0}, U_{2}, V_{0}, V_{2} ; Y_{2} \mid Q\right)
$$$$
-2 \mathbb{I}\left(U_{0}, V_{0} ; Z \mid Q\right)-\mathbb{I}\left(U_{1} ; Z \mid U_{0}, V_{0}\right)-\mathbb{I}\left(U_{1}, U_{2}, V_{1}, V_{2} ; Z \mid U_{0}, V_{0}\right)-\mathbb{I}\left(U_{1}, V_{1} ; U_{2}, V_{2} \mid U_{0}, V_{0}\right)
$$

$$
\begin{aligned}
& 2 R_{1}+2 R_{2}<\mathbb{I}\left(U_{0}, U_{1} ; Y_{1} \mid Q, V_{0}, V_{1}\right)+\mathbb{I}\left(U_{2}, V_{0}, V_{2} ; Y_{2} \mid Q, U_{0}\right)+\mathbb{I}\left(U_{0}, U_{1}, V_{0}, V_{1} ; Y_{1} \mid Q\right) \\
& \quad-2 \mathbb{I}\left(U_{0}, V_{0} ; Z \mid Q\right)-\mathbb{I}\left(U_{1} ; Z \mid U_{0}, V_{0}\right)-\mathbb{I}\left(U_{1}, U_{2}, V_{1}, V_{2} ; Z \mid U_{0}, V_{0}\right)-\mathbb{I}\left(U_{1}, V_{1} ; U_{2}, V_{2} \mid U_{0}, V_{0}\right)
\end{aligned}
$$

$$
\begin{gathered}
2 R_{1}+2 R_{2}<\mathbb{I}\left(U_{0}, U_{2} ; Y_{2} \mid Q, V_{0}, V_{2}\right)+2 \mathbb{I}\left(V_{0}, V_{1} ; Y_{1} \mid Q, U_{0}, U_{1}\right)+\mathbb{I}\left(U_{0}, U_{1}, V_{1} ; Y_{1} \mid Q, V_{0}\right) \\
-2 \mathbb{I}\left(U_{0}, V_{0} ; Z \mid Q\right)-2 \mathbb{I}\left(V_{1} ; Z \mid U_{0}, V_{0}\right)-\mathbb{I}\left(U_{1}, U_{2}, V_{1} ; Z \mid U_{0}, V_{0}\right)-\mathbb{I}\left(U_{1} ; U_{2} \mid U_{0}\right)
\end{gathered}
$$

$$
2 R_{1}+2 R_{2}<2 \mathbb{I}\left(U_{0}, U_{2} ; Y_{2} \mid Q, V_{0}, V_{2}\right)+\mathbb{I}\left(V_{0}, V_{1} ; Y_{1} \mid Q, U_{0}, U_{1}\right)+\mathbb{I}\left(U_{2}, V_{0}, V_{2} ; Y_{2} \mid Q, U_{0}\right)
$$$$
-2 \mathbb{I}\left(U_{0}, V_{0} ; Z \mid Q\right)-2 \mathbb{I}\left(U_{2} ; Z \mid U_{0}, V_{0}\right)-\mathbb{I}\left(U_{2}, V_{1}, V_{2}: Z \mid U_{0}, V_{0}\right)-\mathbb{I}\left(V_{1} ; V_{2} \mid V_{0}\right)
$$

$$
\begin{aligned}
& 2 R_{1}+2 R_{2}<2 \mathbb{I}\left(U_{0}, U_{2} ; Y_{2} \mid Q, V_{0}, V_{2}\right)+\mathbb{I}\left(U_{1}, V_{0}, V_{1} ; Y_{1} \mid Q, U_{0}\right)+\mathbb{I}\left(U_{2}, V_{0}, V_{2} ; Y_{2} \mid Q, U_{0}\right) \\
& \quad-2 \mathbb{I}\left(U_{0}, V_{0} ; Z \mid Q\right)-2 \mathbb{I}\left(U_{2} ; Z \mid U_{0}, V_{0}\right)-\mathbb{I}\left(U_{1}, U_{2}, V_{1}, V_{2} ; Z \mid U_{0}, V_{0}\right)-\mathbb{I}\left(U_{1}, V_{1} ; U_{2}, V_{2} \mid U_{0}, V_{0}\right)
\end{aligned}
$$

$$
\begin{gathered}
2 R_{1}+2 R_{2}<\mathbb{I}\left(U_{0}, U_{2} ; Y_{2} \mid Q, V_{0}, V_{2}\right)+\mathbb{I}\left(V_{0}, V_{1} ; Y_{1} \mid Q, U_{0}, U_{1}\right)+\mathbb{I}\left(U_{0}, U_{1}, V_{0}, V_{1} ; Y_{1} \mid Q\right) \\
-2 \mathbb{I}\left(U_{0}, V_{0} ; Z \mid Q\right)-\mathbb{I}\left(V_{1} ; Z \mid U_{0}, V_{0}\right)-\mathbb{I}\left(U_{1}, U_{2}, V_{1} ; Z \mid U_{0}, V_{0}\right)-\mathbb{I}\left(U_{1} ; U_{2} \mid U_{0}\right)
\end{gathered}
$$$$
2 R_{1}+2 R_{2}<\mathbb{I}\left(U_{0}, U_{2} ; Y_{2} \mid Q, V_{0}, V_{2}\right)+\mathbb{I}\left(V_{0}, V_{1} ; Y_{1} \mid Q, U_{0}, U_{1}\right)+\mathbb{I}\left(U_{0}, U_{2}, V_{0}, V_{2} ; Y_{2} \mid Q\right)
$$$$
-2 \mathbb{I}\left(U_{0}, V_{0} ; Z \mid Q\right)-\mathbb{I}\left(U_{2} ; Z \mid U_{0}, V_{0}\right)-\mathbb{I}\left(U_{2}, V_{1}, V_{2} ; Z \mid U_{0}, V_{0}\right)-\mathbb{I}\left(V_{1} ; V_{2} \mid V_{0}\right)
$$$$
2 R_{1}+2 R_{2}<\mathbb{I}\left(U_{0}, U_{2} ; Y_{2} \mid Q, V_{0}, V_{2}\right)+\mathbb{I}\left(U_{1}, V_{0}, V_{1} ; Y_{1} \mid Q, U_{0}\right)+\mathbb{I}\left(U_{0}, U_{2}, V_{0}, V_{2} ; Y_{2} \mid Q\right)
$$$$
-2 \mathbb{I}\left(U_{0}, V_{0} ; Z \mid Q\right)-\mathbb{I}\left(U_{2} ; Z \mid U_{0}, V_{0}\right)-\mathbb{I}\left(U_{1}, U_{2}, V_{1}, V_{2} ; Z \mid U_{0}, V_{0}\right)-\mathbb{I}\left(U_{1}, V_{1} ; U_{2}, V_{2} \mid U_{0}, V_{0}\right)
$$$$
2 R_{1}+2 R_{2}<\mathbb{I}\left(U_{0}, U_{2} ; Y_{2} \mid Q, V_{0}, V_{2}\right)+\mathbb{I}\left(U_{2}, V_{0}, V_{2} ; Y_{2} \mid Q, U_{0}\right)+\mathbb{I}\left(U_{0}, U_{1}, V_{0}, V_{1} ; Y_{1} \mid Q\right)
$$$$
-2 \mathbb{I}\left(U_{0}, V_{0} ; Z \mid Q\right)-\mathbb{I}\left(U_{2} ; Z \mid U_{0}, V_{0}\right)-\mathbb{I}\left(U_{1}, U_{2}, V_{1}, V_{2} ; Z \mid U_{0}, V_{0}\right)-\mathbb{I}\left(U_{1}, V_{1} ; U_{2}, V_{2} \mid U_{0}, V_{0}\right)
$$ 


$$
\begin{aligned}
& 2 R_{1}+2 R_{2}<2 \mathbb{I}\left(V_{0}, V_{1} ; Y_{1} \mid Q, U_{0}, U_{1}\right)+\mathbb{I}\left(U_{0}, U_{1}, V_{1} ; Y_{1} \mid Q, V_{0}\right)+\mathbb{I}\left(U_{0}, U_{2}, V_{2} ; Y_{2} \mid Q, V_{0}\right) \\
& -2 \mathbb{I}\left(U_{0}, V_{0} ; Z \mid Q\right)-2 \mathbb{I}\left(V_{1} ; Z \mid U_{0}, V_{0}\right)-\mathbb{I}\left(U_{1}, U_{2}, V_{1}, V_{2} ; Z \mid U_{0}, V_{0}\right)-\mathbb{I}\left(U_{1}, V_{1} ; U_{2}, V_{2} \mid U_{0}, V_{0}\right) \\
& 2 R_{1}+2 R_{2}<\mathbb{I}\left(V_{0}, V_{1} ; Y_{1} \mid Q, U_{0}, U_{1}\right)+\mathbb{I}\left(V_{0}, V_{2} ; Y_{2} \mid Q, U_{0}, U_{2}\right) \\
& +\mathbb{I}\left(U_{0}, U_{1}, V_{1} ; Y_{1} \mid Q, V_{0}\right)+\mathbb{I}\left(U_{0}, U_{2}, V_{2} ; Y_{2} \mid Q, V_{0}\right)-2 \mathbb{I}\left(U_{0}, V_{0} ; Z \mid Q\right) \\
& -\mathbb{I}\left(V_{1}, V_{2} ; Z \mid U_{0}, V_{0}\right)-\mathbb{I}\left(U_{1}, U_{2}, V_{1}, V_{2} ; Z \mid U_{0}, V_{0}\right)-\mathbb{I}\left(V_{1} ; V_{2} \mid V_{0}\right)-\mathbb{I}\left(U_{1}, V_{1} ; U_{2}, V_{2} \mid U_{0}, V_{0}\right) \\
& 2 R_{1}+2 R_{2}<\mathbb{I}\left(V_{0}, V_{1} ; Y_{1} \mid Q, U_{0}, U_{1}\right)+\mathbb{I}\left(U_{0}, U_{1}, V_{1} ; Y_{1} \mid Q, V_{0}\right)+\mathbb{I}\left(U_{0}, U_{2}, V_{0}, V_{2} ; Y_{2} \mid Q\right) \\
& -2 \mathbb{I}\left(U_{0}, V_{0} ; Z \mid Q\right)-\mathbb{I}\left(V_{1} ; Z \mid U_{0}, V_{0}\right)-\mathbb{I}\left(U_{1}, U_{2}, V_{1}, V_{2} ; Z \mid U_{0}, V_{0}\right)-\mathbb{I}\left(U_{1}, V_{1} ; U_{2}, V_{2} \mid U_{0}, V_{0}\right) \\
& 2 R_{1}+2 R_{2}<\mathbb{I}\left(V_{0}, V_{1} ; Y_{1} \mid Q, U_{0}, U_{1}\right)+\mathbb{I}\left(U_{0}, U_{2}, V_{2} ; Y_{2} \mid Q, V_{0}\right)+\mathbb{I}\left(U_{0}, U_{1}, V_{0}, V_{1} ; Y_{1} \mid Q\right) \\
& -2 \mathbb{I}\left(U_{0}, V_{0} ; Z \mid Q\right)-\mathbb{I}\left(V_{1} ; Z \mid U_{0}, V_{0}\right)-\mathbb{I}\left(U_{1}, U_{2}, V_{1}, V_{2} ; Z \mid U_{0}, V_{0}\right)-\mathbb{I}\left(U_{1}, V_{1} ; U_{2}, V_{2} \mid U_{0}, V_{0}\right) \\
& 2 R_{1}+2 R_{2}<2 \mathbb{I}\left(V_{0}, V_{2} ; Y_{2} \mid Q, U_{0}, U_{2}\right)+\mathbb{I}\left(U_{0}, U_{1}, V_{1} ; Y_{1} \mid Q, V_{0}\right)+\mathbb{I}\left(U_{0}, U_{2}, V_{2} ; Y_{2} \mid Q, V_{0}\right) \\
& -2 \mathbb{I}\left(U_{0}, V_{0} ; Z \mid Q\right)-2 \mathbb{I}\left(V_{2} ; Z \mid U_{0}, V_{0}\right)-\mathbb{I}\left(U_{1}, U_{2}, V_{1}, V_{2} ; Z \mid U_{0}, V_{0}\right)-\mathbb{I}\left(U_{1}, V_{1} ; U_{2}, V_{2} \mid U_{0}, V_{0}\right) \\
& 2 R_{1}+2 R_{2}<\mathbb{I}\left(V_{0}, V_{2} ; Y_{2} \mid Q, U_{0}, U_{2}\right)+\mathbb{I}\left(U_{0}, U_{1}, V_{1} ; Y_{1} \mid Q, V_{0}\right)+\mathbb{I}\left(U_{0}, U_{2}, V_{0}, V_{2} ; Y_{2} \mid Q\right) \\
& -2 \mathbb{I}\left(U_{0}, V_{0} ; Z \mid Q\right)-\mathbb{I}\left(V_{2} ; Z \mid U_{0}, V_{0}\right)-\mathbb{I}\left(U_{1}, U_{2}, V_{1}, V_{2} ; Z \mid U_{0}, V_{0}\right)-\mathbb{I}\left(U_{1}, V_{1} ; U_{2}, V_{2} \mid U_{0}, V_{0}\right) \\
& 2 R_{1}+2 R_{2}<\mathbb{I}\left(V_{0}, V_{2} ; Y_{2} \mid Q, U_{0}, U_{2}\right)+\mathbb{I}\left(U_{0}, U_{2}, V_{2} ; Y_{2} \mid Q, V_{0}\right)+\mathbb{I}\left(U_{0}, U_{1}, V_{0}, V_{1} ; Y_{1} \mid Q\right) \\
& -2 \mathbb{I}\left(U_{0}, V_{0} ; Z \mid Q\right)-\mathbb{I}\left(V_{2} ; Z \mid U_{0}, V_{0}\right)-\mathbb{I}\left(U_{1}, U_{2}, V_{1}, V_{2} ; Z \mid U_{0}, V_{0}\right)-\mathbb{I}\left(U_{1}, V_{1} ; U_{2}, V_{2} \mid U_{0}, V_{0}\right) \\
& 2 R_{1}+2 R_{2}<\mathbb{I}\left(U_{0}, U_{1}, V_{0}, V_{1} ; Y_{1} \mid Q\right)+\mathbb{I}\left(U_{0}, U_{2}, V_{0}, V_{2} ; Y_{2} \mid Q\right) \\
& -2 \mathbb{I}\left(U_{0}, V_{0} ; Z \mid Q\right)-\mathbb{I}\left(U_{1}, U_{2}, V_{1}, V_{2} ; Z \mid U_{0}, V_{0}\right)-\mathbb{I}\left(U_{1}, V_{1} ; U_{2}, V_{2} \mid U_{0}, V_{0}\right) \\
& 3 R_{1}+3 R_{2}<\mathbb{I}\left(U_{0}, U_{1} ; Y_{1} \mid Q, V_{0}, V_{1}\right)+\mathbb{I}\left(U_{1}, V_{0}, V_{1} ; Y_{1} \mid Q, U_{0}\right) \\
& +2 \mathbb{I}\left(U_{0}, U_{2}, V_{0}, V_{2} ; Y_{2} \mid Q\right)-3 \mathbb{I}\left(U_{0}, V_{0} ; Z\right)-\mathbb{I}\left(U_{1}, U_{2}, V_{2} ; Z \mid U_{0}, V_{0}\right) \\
& -\mathbb{I}\left(U_{1}, U_{2}, V_{1}, V_{2} ; Z \mid U_{0}, V_{0}\right)-\mathbb{I}\left(U_{1} ; U_{2} \mid U_{0}\right)-\mathbb{I}\left(U_{1}, V_{1} ; U_{2}, V_{2} \mid U_{0}, V_{0}\right) \\
& 3 R_{1}+3 R_{2}<\mathbb{I}\left(U_{0}, U_{2} ; Y_{2} \mid Q, V_{0}, V_{2}\right)+\mathbb{I}\left(U_{2}, V_{0}, V_{2} ; Y_{2} \mid Q, U_{0}\right) \\
& +2 \mathbb{I}\left(U_{0}, U_{1}, V_{0}, V_{1} ; Y_{1} \mid Q\right)-3 \mathbb{I}\left(U_{0}, V_{0} ; Z \mid Q\right)-\mathbb{I}\left(U_{1}, U_{2}, V_{1} ; Z \mid U_{0}, V_{0}\right) \\
& -\mathbb{I}\left(U_{1}, U_{2}, V_{1}, V_{2} ; Z \mid U_{0}, V_{0}\right)-\mathbb{I}\left(U_{1} ; U_{2} \mid U_{0}\right)-\mathbb{I}\left(U_{1}, V_{1} ; U_{2}, V_{2} \mid U_{0}, V_{0}\right) \\
& 3 R_{1}+3 R_{2}<\mathbb{I}\left(V_{0}, V_{1} ; Y_{1} \mid Q, U_{0}, U_{1}\right)+\mathbb{I}\left(U_{0}, U_{1}, V_{1} ; Y_{1} \mid Q, V_{0}\right) \\
& +2 \mathbb{I}\left(U_{0}, U_{2}, V_{0}, V_{2} ; Y_{2} \mid Q\right)-3 \mathbb{I}\left(U_{0}, V_{0} ; Z \mid Q\right)-\mathbb{I}\left(U_{2}, V_{1}, V_{2} ; Z \mid U_{0}, V_{0}\right)
\end{aligned}
$$




$$
\begin{aligned}
& -\mathbb{I}\left(U_{1}, U_{2}, V_{1}, V_{2} ; Z \mid U_{0}, V_{0}\right)-\mathbb{I}\left(V_{1} ; V_{2} \mid V_{0}\right)-\mathbb{I}\left(U_{1}, V_{1} ; U_{2}, V_{2} \mid U_{0}, V_{0}\right) \\
& 3 R_{1}+3 R_{2}<\mathbb{I}\left(V_{0}, V_{2} ; Y_{2} \mid Q, U_{0}, U_{2}\right)+\mathbb{I}\left(U_{0}, U_{2}, V_{2} ; Y_{2} \mid Q, V_{0}\right) \\
& +2 \mathbb{I}\left(U_{0}, U_{1}, V_{0}, V_{1} ; Y_{1} \mid Q\right)-3 \mathbb{I}\left(U_{0}, V_{0} ; Z \mid Q\right)-\mathbb{I}\left(U_{1}, V_{1}, V_{2} ; Z \mid U_{0}, V_{0}\right) \\
& -\mathbb{I}\left(U_{1}, U_{2}, V_{1}, V_{2} ; Z \mid U_{0}, V_{0}\right)-\mathbb{I}\left(V_{1} ; V_{2} \mid V_{0}\right)-\mathbb{I}\left(U_{1}, V_{1} ; U_{2}, V_{2} \mid U_{0}, V_{0}\right) \\
& 3 R_{1}+3 R_{2}<\mathbb{I}\left(U_{0}, U_{1}, V_{1} ; Y_{1} \mid Q, V_{0}\right)+\mathbb{I}\left(U_{1}, V_{0}, V_{1} ; Y_{1} \mid Q, U_{0}\right)+2 \mathbb{I}\left(U_{0}, U_{2}, V_{0}, V_{2} ; Y_{2} \mid Q\right) \\
& -3 \mathbb{I}\left(U_{0}, V_{0} ; Z \mid Q\right)-2 \mathbb{I}\left(U_{1}, U_{2}, V_{1}, V_{2} ; Z \mid U_{0}, V_{0}\right)-2 \mathbb{I}\left(U_{1}, V_{1} ; U_{2}, V_{2} \mid U_{0}, V_{0}\right) \\
& 3 R_{1}+3 R_{2}<\mathbb{I}\left(U_{0}, U_{2}, V_{2} ; Y_{2} \mid Q, V_{0}\right)+\mathbb{I}\left(U_{2}, V_{0}, V_{2} ; Y_{2} \mid Q, U_{0}\right)+2 \mathbb{I}\left(U_{0}, U_{1}, V_{0}, V_{1} ; Y_{1} \mid Q\right) \\
& -3 \mathbb{I}\left(U_{0}, V_{0} ; Z \mid Q\right)-2 \mathbb{I}\left(U_{1}, U_{2}, V_{1}, V_{2} ; Z \mid U_{0}, V_{0}\right)-2 \mathbb{I}\left(U_{1}, V_{1} ; U_{2}, V_{2} \mid U_{0}, V_{0}\right) \\
& 4 R_{1}+4 R_{2}<\mathbb{I}\left(U_{0}, U_{1} ; Y_{1} \mid V_{0}, V_{1}\right)+\mathbb{I}\left(U_{0}, U_{2} ; Y_{2} \mid Q, V_{0}, V_{2}\right)+2 \mathbb{I}\left(U_{1}, V_{0}, V_{1} ; Y_{1} \mid Q, U_{0}\right) \\
& +2 \mathbb{I}\left(U_{0}, U_{2}, V_{0}, V_{2} ; Y_{2} \mid Q\right)-4 \mathbb{I}\left(U_{0}, V_{0} ; Z \mid Q\right)-\mathbb{I}\left(U_{1}, U_{2} ; Z \mid U_{0}, V_{0}\right) \\
& -2 \mathbb{I}\left(U_{1}, U_{2}, V_{1}, V_{2} ; Z \mid U_{0}, V_{0}\right)-\mathbb{I}\left(U_{1} ; U_{2} \mid U_{0}\right)-2 \mathbb{I}\left(U_{1}, V_{1} ; U_{2}, V_{2} \mid U_{0}, V_{0}\right) \\
& 4 R_{1}+4 R_{2}<\mathbb{I}\left(U_{0}, U_{1} ; Y_{1} \mid Q, V_{0}, V_{1}\right)+\mathbb{I}\left(U_{0}, U_{2} ; Y_{2} \mid Q, V_{0}, V_{2}\right) \\
& +2 \mathbb{I}\left(U_{2}, V_{0}, V_{2} ; Y_{2} \mid Q, U_{0}\right)+2 \mathbb{I}\left(U_{0}, U_{1}, V_{0}, V_{1} ; Y_{1} \mid Q\right)-4 \mathbb{I}\left(U_{0}, V_{0} ; Z \mid Q\right) \\
& -\mathbb{I}\left(U_{1}, U_{2} ; Z \mid U_{0}, V_{0}\right)-2 \mathbb{I}\left(U_{1}, U_{2}, V_{1}, V_{2} ; Z \mid U_{0}, V_{0}\right)-\mathbb{I}\left(U_{1} ; U_{2} \mid U_{0}\right)-2 \mathbb{I}\left(U_{1}, V_{1} ; U_{2}, V_{2} \mid U_{0}, V_{0}\right) \\
& 4 R_{1}+4 R_{2}<\mathbb{I}\left(V_{0}, V_{1} ; Y_{1} \mid Q, U_{0}, U_{1}\right)+\mathbb{I}\left(V_{0}, V_{2} ; Y_{2} \mid Q, U_{0}, U_{2}\right) \\
& +2 \mathbb{I}\left(U_{0}, U_{1}, V_{1} ; Y_{1} \mid Q, V_{0}\right)+2 \mathbb{I}\left(U_{0}, U_{2}, V_{0}, V_{2} ; Y_{2} \mid Q\right)-4 \mathbb{I}\left(U_{0}, V_{0} ; Z \mid Q\right) \\
& -\mathbb{I}\left(V_{1}, V_{2} ; Z \mid U_{0}, V_{0}\right)-2 \mathbb{I}\left(U_{1}, U_{2}, V_{1}, V_{2} ; Z \mid U_{0}, V_{0}\right)-\mathbb{I}\left(V_{1} ; V_{2} \mid V_{0}\right)-2 \mathbb{I}\left(U_{1}, V_{1} ; U_{2}, V_{2} \mid U_{0}, V_{0}\right) \\
& 4 R_{1}+4 R_{2}<\mathbb{I}\left(V_{0}, V_{1} ; Y_{1} \mid Q, U_{0}, U_{1}\right)+\mathbb{I}\left(V_{0}, V_{2} ; Y_{2} \mid Q, U_{0}, U_{2}\right) \\
& +2 \mathbb{I}\left(U_{0}, U_{2}, V_{2} ; Y_{2} \mid Q, V_{0}\right)+2 \mathbb{I}\left(U_{0}, U_{1}, V_{0}, V_{1} ; Y_{1} \mid Q\right)-4 \mathbb{I}\left(U_{0}, V_{0} ; Z \mid Q\right) \\
& -\mathbb{I}\left(V_{1}, V_{2} ; Z \mid U_{0}, V_{0}\right)-2 \mathbb{I}\left(U_{1}, U_{2}, V_{1}, V_{2} ; Z \mid U_{0}, V_{0}\right)-\mathbb{I}\left(V_{1} ; V_{2} \mid V_{0}\right)-2 \mathbb{I}\left(U_{1}, V_{1} ; U_{2}, V_{2} \mid U_{0}, V_{0}\right)
\end{aligned}
$$

for some distribution

$$
p(q) p\left(u_{0}, u_{1}, u_{2} \mid q\right) p\left(v_{0}, v_{1}, v_{2} \mid q\right) p\left(x_{1} \mid u_{0}, u_{1}, u_{2}\right) p\left(x_{2} \mid v_{0}, v_{1}, v_{2}\right) p\left(y_{1}, y_{2}, z \mid x_{1}, x_{2}\right)
$$

Proof. The achievability proof is inspired by [9], and is based on solving a dual secret key agreement problem in the source model that includes shared randomness at all terminals (see Fig. 6). In this dual model, rate constraints are derived so that the input and output distributions of the dual model approximate that of the original model while satisfying reliability and secrecy 


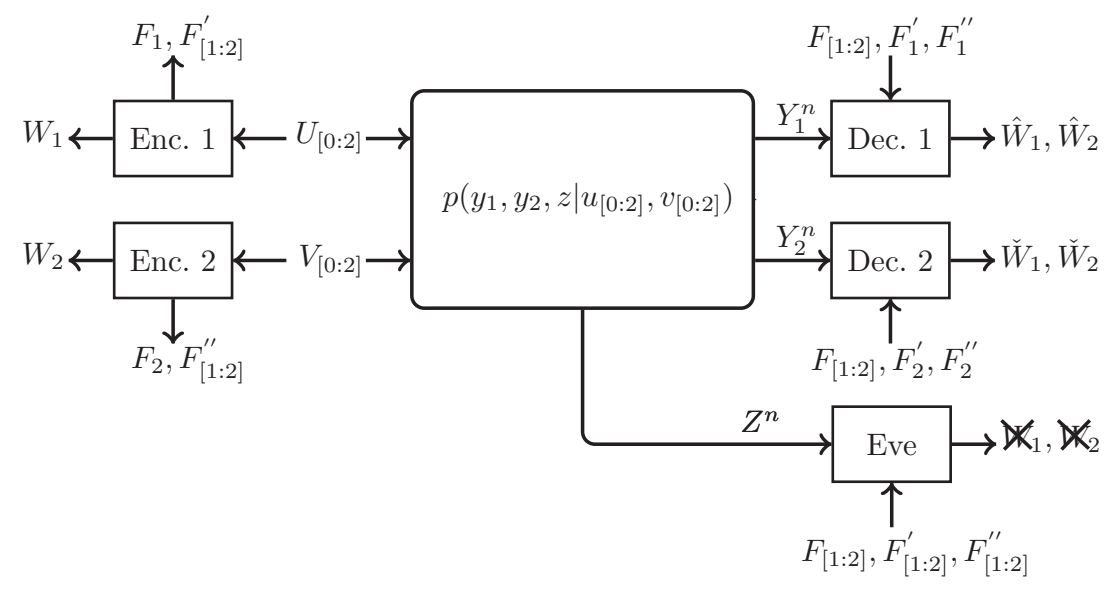

Fig. 6. Dual secret key agreement problem in the source model for the original problem.

conditions in the dual model. The probability approximation then guarantees that reliability and secrecy conditions can be achieved in the original model. Finally, it is shown that there exists one realization of shared randomness for which the above mentioned are valid, thus removing the necessity for common randomness.

We begin by developing the encoding and decoding strategies for the source model and the original model, and derive and compare the joint probability distributions arising from these two strategies.

We begin with the multi-terminal secret key agreement problem in the source model as depicted in Fig. 6. Let $\left(U_{[0: 2]}^{n}, V_{[0: 2]}^{n}, X_{1}^{n}, X_{2}^{n}, Y_{1}^{n}, Y_{2}^{n}, Z^{n}\right)$ be i.i.d. and distributed according to

$$
p\left(u_{[0: 2]}, x_{1}\right) p\left(v_{[0: 2]}, x_{2}\right) p\left(y_{1}, y_{2}, z \mid x_{1}, x_{2}\right) .
$$

\section{Random Binning:}

- To each and every $u_{0}^{n}$, uniformly and independently assign two random bin indices $w_{1} \in$ $\llbracket 1,2^{n R_{1}} \rrbracket$ and $f_{1} \in \llbracket 1,2^{n \tilde{R}_{1}} \rrbracket$.

- To each pair $\left(u_{0}^{n}, u_{j}^{n}\right)$ for $j=1,2$ uniformly and independently assign random bin index $f_{j}^{\prime} \in \llbracket 1,2^{n \tilde{R}_{j}^{\prime}} \rrbracket$.

- To each $v_{0}^{n}$ uniformly and independently assign two random bin indices $w_{2} \in \llbracket 1,2^{n R_{2}} \rrbracket$ and $f_{2} \in \llbracket 1,2^{n \tilde{R}_{2}} \rrbracket$.

- To each pair $\left(v_{0}^{n}, v_{j}^{n}\right)$ for $j=1,2$ uniformly and independently assign random bin index $f_{j}^{\prime \prime} \in \llbracket 1,2^{n \tilde{R}_{j}^{\prime \prime}} \rrbracket$. 
- The random variables representing bin indices are:

$$
W_{[1: 2]}, \quad F_{[1: 2]}, \quad F_{[1: 2]}^{\prime}, \quad F_{[1: 2]}^{\prime \prime} .
$$

- Decoder 1 is a Slepian-Wolf decoder observing $\left(y_{1}^{n}, f_{[1: 2]}, f_{1}^{\prime}, f_{1}^{\prime \prime}\right)$, and producing $\left(\hat{u}_{0}^{n}, \hat{u}_{1}^{n}\right)$ and $\left(\hat{v}_{0}^{n}, \hat{v}_{1}^{n}\right)$, thus declaring $\hat{w}_{1}=W_{1}\left(\hat{u}_{0}^{n}\right)$ and $\hat{w}_{2}=W_{2}\left(\hat{v}_{0}^{n}\right)$ to be the estimate of the pair $\left(w_{1}, w_{2}\right)$.

- Decoder 2 is a Slepian-Wolf decoder observing $\left(y_{2}^{n}, f_{[1: 2]}, f_{2}^{\prime}, f_{2}^{\prime \prime}\right)$, and producing $\left(\check{u}_{0}^{n}, \breve{u}_{2}^{n}\right)$ and $\left(\check{v}_{0}^{n}, \breve{v}_{2}^{n}\right)$, thus declaring the bin indices $\check{w}_{1}=W_{1}\left(\check{u}_{0}^{n}\right)$ and $\check{w}_{2}=W_{2}\left(\check{v}_{0}^{n}\right)$ as the estimate of the pair $\left(w_{1}, w_{2}\right)$.

To condense the notation, we define the following variables:

$$
\begin{gathered}
\mathbf{f} \triangleq\left(f_{[1: 2]}, f_{[1: 2]}^{\prime}, f_{[1: 2]}^{\prime \prime}\right), \\
\hat{\mathbf{u}} \triangleq\left(\hat{u}_{0}^{n}, \check{u}_{0}^{n}, \hat{u}_{1}^{n}, \check{u}_{2}^{n}, \hat{v}_{0}^{n}, \check{v}_{0}^{n}, \hat{v}_{1}^{n}, \check{v}_{2}^{n}\right),
\end{gathered}
$$

The random pmf induced by random binning is then as follows:

$$
\begin{aligned}
& P\left(u_{[0: 2]}^{n}, v_{[0: 2]}^{n}, x_{[1: 2]}^{n}, y_{1}^{n}, y_{2}^{n}, z^{n}, w_{[1: 2]}, \mathbf{f}, \hat{\mathbf{u}}\right) \\
& =p\left(u_{[0: 2]}^{n}, v_{[0: 2]}^{n}, x_{[1: 2]}^{n}, y_{1}^{n}, y_{2}^{n}, z^{n}\right) P\left(w_{[1: 2]}, f_{[1: 2]} \mid u_{0}^{n}, v_{0}^{n}\right) P\left(f_{[1: 2]}^{\prime}, f_{[1: 2]}^{\prime \prime} \mid u_{[0: 2]}^{n}, v_{[0: 2]}^{n}\right) \\
& \times P^{S W}\left(\hat{u}_{0}^{n}, \hat{u}_{1}^{n}, \hat{v}_{0}^{n}, \hat{v}_{1}^{n} \mid y_{1}^{n}, f_{[1: 2]}, f_{1}^{\prime}, f_{1}^{\prime \prime}\right) P^{S W}\left(\check{u}_{0}^{n}, \check{u}_{2}^{n}, \check{v}_{0}^{n}, \check{v}_{2}^{n} \mid y_{2}^{n}, f_{[1: 2]}, f_{2}^{\prime}, f_{2}^{\prime \prime}\right) \\
& =P\left(w_{[1: 2]}, f_{[1: 2]}, u_{0}^{n}, v_{0}^{n}\right) P\left(f_{[1: 2]}^{\prime}, f_{[1: 2]}^{\prime \prime}, u_{[1: 2]}^{n}, v_{[1: 2]}^{n} \mid u_{0}^{n}, v_{0}^{n}\right) \\
& \times p\left(x_{1}^{n} \mid u_{[0: 2]}^{n}\right) p\left(x_{2}^{n} \mid v_{[0: 2]}^{n}\right) p\left(y_{1}^{n}, y_{2}^{n}, z^{n} \mid x_{1}^{n}, x_{2}^{n}\right) \\
& \times P^{S W}\left(\hat{u}_{0}^{n}, \hat{u}_{1}^{n}, \hat{v}_{0}^{n}, \hat{v}_{1}^{n} \mid y_{1}^{n}, f_{[1: 2]}, f_{1}^{\prime}, f_{1}^{\prime \prime}\right) P^{S W}\left(\check{u}_{0}^{n}, \check{u}_{2}^{n}, \check{v}_{0}^{n}, \check{v}_{2}^{n} \mid y_{2}^{n}, f_{[1: 2]}, f_{2}^{\prime}, f_{2}^{\prime \prime}\right) \\
& =P\left(w_{[1: 2]}, f_{[1: 2]}\right) P\left(u_{0}^{n}, v_{0}^{n} \mid w_{[1: 2]}, f_{[1: 2]}\right) P\left(f_{[1: 2]}^{\prime}, f_{[1: 2]}^{\prime \prime} \mid u_{0}^{n}, v_{0}^{n}\right) P\left(u_{[1: 2]}^{n}, v_{[1: 2]}^{n} \mid u_{0}^{n}, v_{0}^{n}, f_{[1: 2]}^{\prime}, f_{[1: 2]}^{\prime \prime}\right) \\
& \times p\left(x_{1}^{n} \mid u_{[0: 2]}^{n}\right) p\left(x_{2}^{n} \mid v_{[0: 2]}^{n}\right) p\left(y_{1}^{n}, y_{2}^{n}, z^{n} \mid x_{1}^{n}, x_{2}^{n}\right) \\
& \times P^{S W}\left(\hat{u}_{0}^{n}, \hat{u}_{1}^{n}, \hat{v}_{0}^{n}, \hat{v}_{1}^{n} \mid y_{1}^{n}, f_{[1: 2]}, f_{1}^{\prime}, f_{1}^{\prime \prime}\right) P^{S W}\left(\check{u}_{0}^{n}, \check{u}_{2}^{n}, \check{v}_{0}^{n}, \check{v}_{2}^{n} \mid y_{2}^{n}, f_{[1: 2]}, f_{2}^{\prime}, f_{2}^{\prime \prime}\right) .
\end{aligned}
$$

$P^{S W}$ denotes the pmf of the output of the Slepian-Wolf decoder, which is a random pmf. $\hat{W}_{1}, \hat{W}_{2}$ and $\check{W}_{1}, \check{W}_{2}$ are omitted because they are functions of other random variables.

We now return to the original problem illustrated in Fig. 1 except that, in addition, a genie provides all terminals with shared randomness described by $\left(F_{[1: 2]}, F_{[1: 2]}^{\prime}, F_{[1: 2]}^{\prime \prime}\right)$, whose distribution will be clarified in the sequel. In this augmented model: 
- The messages $W_{1}, W_{2}$ are mutually independent and uniformly distributed with rates $R_{1}, R_{2}$ respectively. The shared randomness $\left(F_{1}, F_{2}\right)$ is uniformly distributed over $\llbracket 1,2^{n \tilde{R}_{1}} \rrbracket$, $\llbracket 1,2^{n \tilde{R}_{2}} \rrbracket$, and independent of $W_{1}, W_{2}$.

- Encoder 1 and 2 are stochastic encoders producing codewords $U_{0}^{n}, V_{0}^{n}$ according to distributions $P\left(u_{0}^{n} \mid w_{[1: 2]}, f_{[1: 2]}\right)$ and $P\left(v_{0}^{n} \mid w_{[1: 2]}, f_{[1: 2]}\right)$, respectively, which are the marginals of distribution $P\left(u_{0}^{n}, v_{0}^{n} \mid w_{[1: 2]}, f_{[1: 2]}\right)$ appearing in (38). This choice of encoder establishes a the connection between the two models.

- The four random variables $F_{[1: 2]}^{\prime}, F_{[1: 2]}^{\prime \prime}$ are mutually independent and uniformly distributed over, respectively, $\llbracket 1,2^{n \tilde{R}_{1}^{\prime}} \rrbracket$ and $\llbracket 1,2^{n \tilde{R}_{2}^{\prime}} \rrbracket, \llbracket 1,2^{n \tilde{R}_{1}^{\prime \prime}} \rrbracket$ and $\llbracket 1,2^{n \tilde{R}_{2}^{\prime \prime}} \rrbracket$. They are also independent of $\left(U_{0}^{n}, V_{0}^{n}\right)$ and therefore are independent of $\left(W_{[1: 2]}, F_{[1: 2]}\right)$.

- Encoder 1 and 2 further generate $U_{[1: 2]}^{n}, V_{[1: 2]}^{n}$ according to $P\left(u_{[1: 2]}^{n} \mid u_{0}^{n}, v_{0}^{n}, f_{[1: 2]}^{\prime}, f_{[1: 2]}^{\prime \prime}\right)$ and $P\left(v_{[1: 2]}^{n} \mid u_{0}^{n}, v_{0}^{n}, f_{[1: 2]}^{\prime}, f_{[1: 2]}^{\prime \prime}\right)$, respectively, which are marginal distributions of $P\left(u_{[1: 2]}^{n}, v_{[1: 2]}^{n} \mid u_{0}^{n}, v_{0}^{n}, f_{[1: 2]}^{\prime}, f_{[1: 2]}^{\prime \prime}\right)$ from (38).

- Encoder 1 generates $X_{1}^{n}$ i.i.d. according to $p\left(x_{1} \mid u_{[0: 2]}\right)$. Encoder 2 generates $X_{2}^{n}$ i.i.d. according to $p\left(x_{2} \mid v_{[0: 2]}\right) . X_{1}, X_{2}$ are transmitted over the channel.

- Decoders 1 and 2 are Slepian-Wolf decoders inherited from the source model secret key agreement problem, observing respectively $\left(y_{1}^{n}, f_{[1: 2]}, f_{1}^{\prime}, f_{1}^{\prime \prime}\right)$ and $\left(y_{2}^{n}, f_{[1: 2]}, f_{2}^{\prime}, f_{2}^{\prime \prime}\right)$, and producing $\left(\hat{u}_{0}^{n}, \hat{u}_{1}^{n}, \hat{v}_{0}^{n}, \hat{v}_{1}^{n}\right)$ and $\left(\check{u}_{0}^{n}, \check{u}_{2}^{n}, \check{v}_{0}^{n}, \check{v}_{2}^{n}\right)$. Therefore the following random PMFs for the decoder output distributions are inherited from the source model:

$$
\begin{aligned}
& P^{S W}\left(\hat{u}_{0}^{n}, \hat{u}_{1}^{n}, \hat{v}_{0}^{n}, \hat{v}_{1}^{n} \mid y_{1}^{n}, f_{[1: 2]}, f_{1}^{\prime}, f_{1}^{\prime \prime}\right) \\
& P^{S W}\left(\check{u}_{0}^{n}, \check{u}_{2}^{n}, \check{v}_{0}^{n}, \check{v}_{2}^{n} \mid y_{2}^{n}, f_{[1: 2]}, f_{2}^{\prime}, f_{2}^{\prime \prime}\right)
\end{aligned}
$$

- Decoders 1 and 2 then produce estimates of $\left(W_{1}, W_{2}\right)$, which are denoted $\left(\hat{W}_{1}, \hat{W}_{2}\right)$ and $\left(\breve{W}_{1}, \breve{W}_{2}\right)$ respectively.

The random pmf induced by the random binning and the encoding/decoding strategy is as follows:

$$
\begin{aligned}
& \hat{P}\left(u_{[0: 2]}^{n}, v_{[0: 2]}^{n}, y_{1}^{n}, y_{2}^{n}, z^{n}, w_{[1: 2]}, \mathbf{f}, \hat{\mathbf{u}}\right) \\
& =p^{U}\left(w_{[1: 2]}\right) p^{U}\left(f_{[1: 2]}\right) P\left(u_{0}^{n}, v_{0}^{n} \mid w_{[1: 2]}, f_{[1: 2]}\right) p^{U}\left(f_{[1: 2]}^{\prime}\right) p^{U}\left(f_{[1: 2]}^{\prime \prime}\right) P\left(u_{[1: 2]}^{n}, v_{[1: 2]}^{n} \mid u_{0}^{n}, v_{0}^{n}, f_{[1: 2]}^{\prime}, f_{[1: 2]}^{\prime \prime}\right) \\
& \times p\left(x_{1}^{n} \mid u_{[0: 2]}^{n}\right) p\left(x_{2}^{n} \mid v_{[0: 2]}^{n}\right) p\left(y_{1}^{n}, y_{2}^{n}, z^{n} \mid x_{1}^{n}, x_{2}^{n}\right) \\
& \times P^{S W}\left(\hat{u}_{0}^{n}, \hat{u}_{1}^{n}, \hat{v}_{0}^{n}, \hat{v}_{1}^{n} \mid y_{1}^{n}, f_{[1: 2]}, f_{1}^{\prime}, f_{1}^{\prime \prime}\right) P^{S W}\left(\check{u}_{0}^{n}, \check{u}_{2}^{n}, \check{v}_{0}^{n}, \check{v}_{2}^{n} \mid y_{2}^{n}, f_{[1: 2]}, f_{2}^{\prime}, f_{2}^{\prime \prime}\right),
\end{aligned}
$$

where $\mathbf{f}$ and $\hat{\mathbf{u}}$ are defined in (36) and (37), respectively, and $p^{U}$ is the uniform distribution. 
We now find constraints that ensure that the pmfs $\hat{P}$ and $P$ are close in total variation distance. For the source model secret key agreement problem, substituting $X_{1}=X_{2} \leftarrow U_{0}$, and $X_{3}=$ $X_{4} \leftarrow V_{0}$, in [9. Theorem 1] implies that $W_{[1: 2]}$ is nearly independent of $F_{[1: 2]}$ and $Z^{n}$, if

$$
\begin{aligned}
R_{1}+\tilde{R}_{1} & \leq \mathbb{H}\left(U_{0} \mid Z\right), \\
R_{2}+\tilde{R}_{2} & \leq \mathbb{H}\left(V_{0} \mid Z\right), \\
R_{1}+\tilde{R}_{1}+R_{2}+\tilde{R}_{2} & \leq \mathbb{H}\left(U_{0}, V_{0} \mid Z\right),
\end{aligned}
$$

note that [9. Theorem 1] returns a total of 15 inequalities, but the remaining are redundant because of (40)-(42). The above constraints imply that

$$
P\left(z^{n}, w_{[1: 2]}, f_{[1: 2]}\right) \approx p\left(z^{n}\right) p^{U}\left(w_{[1: 2]}\right) p^{U}\left(f_{[1: 2]}\right)
$$

Similarly, substituting $X_{1} \leftarrow\left(U_{0}, U_{1}\right), X_{2} \leftarrow\left(U_{0}, U_{2}\right), X_{3} \leftarrow\left(V_{0}, V_{1}\right), X_{4} \leftarrow\left(V_{0}, V_{2}\right)$, and $Z \leftarrow\left(U_{0}, V_{0}, Z\right)$ in [9, Theorem 1] implies that $\left(f_{[1: 2]}^{\prime}, f_{[1: 2]}^{\prime \prime}\right)$ are nearly mutually independent and independent of $\left(U_{0}, V_{0}, Z\right)$, therefore they are independent of $\left(w_{[1: 2]}, f_{[1: 2]}\right)$, if

$$
\begin{gathered}
\tilde{R}_{j}^{\prime} \leq \mathbb{H}\left(U_{j} \mid U_{0}, V_{0}, Z\right), \\
\tilde{R}_{j}^{\prime \prime} \leq \mathbb{H}\left(V_{j} \mid U_{0}, V_{0}, Z\right), \\
\tilde{R}_{1}^{\prime}+\tilde{R}_{j}^{\prime \prime} \leq \mathbb{H}\left(U_{1}, V_{j} \mid U_{0}, V_{0}, Z\right), \\
\tilde{R}_{2}^{\prime}+\tilde{R}_{j}^{\prime \prime} \leq \mathbb{H}\left(U_{2}, V_{j} \mid U_{0}, V_{0}, Z\right), \\
\tilde{R}_{1}^{\prime}+\tilde{R}_{2}^{\prime} \leq \mathbb{H}\left(U_{1}, U_{2} \mid U_{0}, V_{0}, Z\right), \\
\tilde{R}_{1}^{\prime \prime}+\tilde{R}_{2}^{\prime \prime} \leq \mathbb{H}\left(V_{1}, V_{2} \mid U_{0}, V_{0}, Z\right), \\
\tilde{R}_{1}^{\prime}+\tilde{R}_{2}^{\prime}+\tilde{R}_{j}^{\prime \prime} \leq \mathbb{H}\left(U_{1}, U_{2}, V_{j} \mid U_{0}, V_{0}, Z\right), \\
\tilde{R}_{j}^{\prime}+\tilde{R}_{1}^{\prime \prime}+\tilde{R}_{2}^{\prime \prime} \leq \mathbb{H}\left(U_{j}, V_{1}, V_{2} \mid U_{0}, V_{0}, Z\right), \\
\tilde{R}_{1}^{\prime}+\tilde{R}_{2}^{\prime}+\tilde{R}_{1}^{\prime \prime}+\tilde{R}_{2}^{\prime \prime} \leq \mathbb{H}\left(U_{1}, U_{2}, V_{1}, V_{2} \mid U_{0}, V_{0}, Z\right),
\end{gathered}
$$

for $j=1,2$. The above constraints imply

$$
P\left(z^{n}, u_{0}^{n}, v_{0}^{n}, f_{[1: 2]}^{\prime}, f_{[1: 2]}^{\prime \prime}\right) \approx p\left(z^{n}, u_{0}^{n}, v_{0}^{n}\right) p^{U}\left(f_{[1: 2]}^{\prime}\right) p^{U}\left(f_{[1: 2]}^{\prime \prime}\right) .
$$

Hence,

$$
\begin{gathered}
P\left(w_{[1: 2]}, f_{[1: 2]}\right)=\hat{P}\left(w_{[1: 2]}, f_{[1: 2]}\right)=p^{U}\left(w_{[1: 2]}\right) p^{U}\left(f_{[1: 2]}\right), \\
P\left(f_{[1: 2]}^{\prime}, f_{[1: 2]}^{\prime \prime} \mid u_{0}^{n}, v_{0}^{n}\right)=\hat{P}\left(f_{[1: 2]}^{\prime}, f_{[1: 2]}^{\prime \prime} \mid u_{0}^{n}, v_{0}^{n}\right)=p^{U}\left(f_{[1: 2]}^{\prime}\right) p^{U}\left(f_{[1: 2]}^{\prime \prime}\right) .
\end{gathered}
$$


In other words, the inequalities (40)-(42) and (43)-(51) imply that

$$
P\left(z^{n}, w_{[1: 2]}, f_{[1: 2]}, f_{[1: 2]}^{\prime}, f_{[1: 2]}^{\prime \prime}\right) \approx p\left(z^{n}\right) p^{U}\left(w_{[1: 2]}\right) p^{U}\left(f_{[1: 2]}\right) p^{U}\left(f_{[1: 2]}^{\prime}\right) p^{U}\left(f_{[1: 2]}^{\prime \prime}\right)
$$

Here, the pmf $P\left(z^{n}\right)$ is equal to $p\left(z^{n}\right)$ because the marginal distribution does not include random binning.

Therefore, the distributions in (38) and (39) are nearly equal, that is

$$
P\left(u_{[0: 2]}^{n}, v_{[0: 2]}^{n}, y_{1}^{n}, y_{2}^{n}, z^{n}, w_{[1: 2]}, \mathbf{f}, \hat{\mathbf{u}}\right) \approx \hat{P}\left(u_{[0: 2]}^{n}, v_{[0: 2]}^{n}, y_{1}^{n}, y_{2}^{n}, z^{n}, w_{[1: 2]}, \mathbf{f}, \hat{\mathbf{u}}\right)
$$

Similar to indirect decoding for channel coding it is possible to use indirect decoding for source coding. More precisely, the first and the second decoders only need $\left(u_{0}^{n}, v_{0}^{n}\right)$ to decode $\left(w_{1}, w_{2}\right)$. Decoder 1 and Decoder 2 can indirectly decode $\left(u_{0}^{n}, v_{0}^{n}\right)$ from $\left(y_{1}^{n}, f_{[1: 2]}, f_{1}^{\prime}, f_{1}^{\prime \prime}\right)$ and $\left(y_{2}^{n}, f_{[1: 2]}, f_{2}^{\prime}, f_{2}^{\prime \prime}\right)$, respectively. From [9, Lemma 1] decoding is successful if

$$
\begin{array}{r}
\tilde{R}_{1}+\tilde{R}_{j}^{\prime} \geq \mathbb{H}\left(U_{0}, U_{j} \mid V_{0}, V_{j}, Y_{j}\right), \\
\tilde{R}_{2}+\tilde{R}_{j}^{\prime \prime} \geq \mathbb{H}\left(V_{0}, V_{j} \mid U_{0}, U_{j}, Y_{j}\right), \\
\tilde{R}_{1}+\tilde{R}_{j}^{\prime}+\tilde{R}_{j}^{\prime \prime} \geq \mathbb{H}\left(U_{0}, U_{j}, V_{j} \mid V_{0}, Y_{j}\right), \\
\tilde{R}_{1}+\tilde{R}_{2}+\tilde{R}_{j}^{\prime \prime} \geq \mathbb{H}\left(V_{0}, V_{j} \mid U_{0}, U_{j}, Y_{j}\right), \\
\tilde{R}_{j}^{\prime}+\tilde{R}_{2}+\tilde{R}_{j}^{\prime \prime} \geq \mathbb{H}\left(U_{j}, V_{0}, V_{j} \mid U_{0}, Y_{j}\right), \\
\tilde{R}_{1}+\tilde{R}_{j}^{\prime}+\tilde{R}_{2}+\tilde{R}_{j}^{\prime \prime} \geq \mathbb{H}\left(U_{0}, U_{j}, V_{0}, V_{j} \mid Y_{j}\right),
\end{array}
$$

for $j=1,2$. Note that, inequality (60) is redundant because of (58). It yields

$$
\begin{aligned}
& P\left(u_{[0: 2]}^{n}, v_{[0: 2]}^{n}, y_{1}^{n}, y_{2}^{n}, z^{n}, w_{[1: 2]}, \mathbf{f}, \hat{\mathbf{u}}\right) \approx P\left(u_{[0: 2]}^{n}, v_{[0: 2]}^{n}, y_{1}^{n}, y_{2}^{n}, z^{n}, w_{[1: 2]}, \mathbf{f}\right) \\
& \times \mathbb{1}\left\{\hat{u}_{0}^{n}=\check{u}_{0}^{n}=u_{0}^{n}, \hat{u}_{1}^{n}=u_{1}^{n}, \check{u}_{2}^{n}=u_{2}^{n}\right\} \mathbb{1}\left\{\hat{v}_{0}^{n}=\check{v}_{0}^{n}=v_{0}^{n}, \hat{v}_{1}^{n}=v_{1}^{n}, \check{v}_{2}^{n}=v_{2}^{n}\right\} .
\end{aligned}
$$

From Equations (56), (63), and the triangle inequality,

$$
\begin{aligned}
& \hat{P}\left(u_{[0: 2]}^{n}, v_{[0: 2]}^{n}, y_{1}^{n}, y_{2}^{n}, z^{n}, w_{[1: 2]}, \mathbf{f}, \hat{\mathbf{u}}\right) \approx P\left(u_{[0: 2]}^{n}, v_{[0: 2]}^{n}, y_{1}^{n}, y_{2}^{n}, z^{n}, w_{[1: 2]}, \mathbf{f}\right) \\
& \times \mathbb{1}\left\{\hat{u}_{0}^{n}=\check{u}_{0}^{n}=u_{0}^{n}, \hat{u}_{1}^{n}=u_{1}^{n}, \check{u}_{2}^{n}=u_{2}^{n}\right\} \mathbb{1}\left\{\hat{v}_{0}^{n}=\check{v}_{0}^{n}=v_{0}^{n}, \hat{v}_{1}^{n}=v_{1}^{n}, \check{v}_{2}^{n}=v_{2}^{n}\right\} .
\end{aligned}
$$

For convenience, we reintroduce a lemma from [9]:

Lemma 2. ( [9] Lemma 4]) Consider distributions $p_{X^{n}}, p_{Y^{n} \mid X^{n}}, q_{X^{n}}$, and $q_{Y^{n} \mid X^{n}}$ and random pmfs $P_{X^{n}}, P_{Y^{n} \mid X^{n}}, Q_{X^{n}}$, and $Q_{Y^{n} \mid X^{n}}$. Denoting asymptotic equality under total variation with

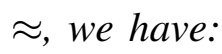


1)

$$
\begin{aligned}
& P_{X^{n}} \approx Q_{X^{n}} \Rightarrow P_{X^{n}} P_{Y^{n} \mid X^{n}} \approx Q_{X^{n}} P_{Y^{n} \mid X^{n}} \\
& P_{X^{n}} P_{Y^{n} \mid X^{n}} \approx Q_{X^{n}} Q_{Y^{n} \mid X^{n}} \Rightarrow P_{X^{n}} \approx Q_{X^{n}}
\end{aligned}
$$

2) If $p_{X^{n}} p_{Y^{n} \mid X^{n}} \approx q_{X^{n}} q_{Y^{n} \mid X^{n}}$, then there exists a sequence $x^{n} \in \mathcal{X}^{n}$ such that

$$
p_{Y^{n} \mid X^{n}=x^{n}} \approx q_{Y^{n} \mid X^{n}=x^{n} .}
$$

3) If $P_{X^{n}} \approx Q_{X^{n}}$ and $P_{X^{n}} P_{Y^{n} \mid X^{n}} \approx P_{X^{n}} Q_{Y^{n} \mid X^{n}}$, then

$$
P_{X^{n}} P_{Y^{n} \mid X^{n}} \approx Q_{X^{n}} Q_{Y^{n} \mid X^{n}}
$$

Using Lemma 2, Equation (66), the marginal distributions of the two sides of (64) are asymptotically equivalent, i.e.,

$$
\begin{aligned}
& \hat{P}\left(u_{[0: 2]}^{n}, v_{[0: 2]}^{n}, z^{n}, w_{[1: 2]}, \mathbf{f}, \hat{\mathbf{u}}\right) \approx P\left(u_{[0: 2]}^{n}, v_{[0: 2]}^{n}, z^{n}, w_{[1: 2]}, \mathbf{f}\right) \\
& \times \mathbb{1}\left\{\hat{u}_{0}^{n}=\check{u}_{0}^{n}=u_{0}^{n}, \hat{u}_{1}^{n}=u_{1}^{n}, \check{u}_{2}^{n}=u_{2}^{n}\right\} \mathbb{1}\left\{\hat{v}_{0}^{n}=\check{v}_{0}^{n}=v_{0}^{n}, \hat{v}_{1}^{n}=v_{1}^{n}, \check{v}_{2}^{n}=v_{2}^{n}\right\} .
\end{aligned}
$$

Using Lemma 2, Equation (65) we multiply the two sides of Equation (69) by the conditional distribution:

$$
\begin{aligned}
& \hat{P}\left(\hat{w}_{1}, \check{w}_{1}, \hat{w}_{2}, \check{w}_{2} \mid u_{[0: 2]}^{n}, v_{[0: 2]}^{n}, z^{n}, w_{[1: 2]}, \mathbf{f}, \hat{\mathbf{u}}\right)= \\
& \quad \mathbb{1}\left\{W_{1}\left(\hat{u}_{0}^{n}\right)=\hat{w}_{1}, W_{1}\left(\check{u}_{0}^{n}\right)=\check{w}_{1}\right\} \mathbb{1}\left\{W_{2}\left(\hat{v}_{0}^{n}\right)=\hat{w}_{2}, W_{2}\left(\check{v}_{0}^{n}\right)=\check{w}_{2}\right\}
\end{aligned}
$$

to get:

$$
\begin{aligned}
& \hat{P}\left(u_{[0: 2]}^{n}, v_{[0: 2]}^{n}, z^{n}, w_{[1: 2]}, \mathbf{f}, \hat{\mathbf{u}}, \hat{w}_{1}, \check{w}_{1}, \hat{w}_{2}, \check{w}_{2}\right) \approx P\left(u_{[0: 2]}^{n}, v_{[0: 2]}^{n}, z^{n}, w_{[1: 2]}, \mathbf{f}\right) \\
& \times \mathbb{1}\left\{\hat{u}_{0}^{n}=\check{u}_{0}^{n}=u_{0}^{n}, \hat{u}_{1}^{n}=u_{1}^{n}, \check{u}_{2}^{n}=u_{2}^{n}\right\} \mathbb{1}\left\{\hat{v}_{0}^{n}=\check{v}_{0}^{n}=v_{0}^{n}, \hat{v}_{1}^{n}=v_{1}^{n}, \check{v}_{2}^{n}=v_{2}^{n}\right\} \\
& \times \mathbb{1}\left\{W_{1}\left(\hat{u}_{0}^{n}\right)=\hat{w}_{1}, W_{1}\left(\check{u}_{0}^{n}\right)=\check{w}_{1}\right\} \mathbb{1}\left\{W_{2}\left(\hat{v}_{0}^{n}\right)=\hat{w}_{2}, W_{2}\left(\check{v}_{0}^{n}\right)=\check{w}_{2}\right\} \\
& =P\left(u_{[0: 2]}^{n}, v_{[0: 2]}^{n}, z^{n}, w_{[1: 2]}, \mathbf{f}\right) \\
& \times \mathbb{1}\left\{\hat{u}_{0}^{n}=\check{u}_{0}^{n}=u_{0}^{n}, \hat{u}_{1}^{n}=u_{1}^{n}, \check{u}_{2}^{n}=u_{2}^{n}\right\} \mathbb{1}\left\{\hat{v}_{0}^{n}=\check{v}_{0}^{n}=v_{0}^{n}, \hat{v}_{1}^{n}=v_{1}^{n}, \check{v}_{2}^{n}=v_{2}^{n}\right\} \\
& \times \mathbb{1}\left\{\hat{w}_{1}=\check{w}_{1}=w_{1}, \hat{w}_{2}=\check{w}_{2}=w_{2}\right\},
\end{aligned}
$$

where $W_{1}\left(u_{0}^{n}\right)=\hat{w}_{1}$ and $W_{2}\left(v_{0}^{n}\right)=\hat{w}_{2}$ denote the bins assigned to $u_{0}^{n}$ and $v_{0}^{n}$, respectively. Using (70) and Lemma 2, Equation (65) leads to

$$
\hat{P}\left(z^{n}, w_{[1: 2]}, \mathbf{f}, \hat{w}_{1}, \check{w}_{1}, \hat{w}_{2}, \check{w}_{2}\right) \approx P\left(z^{n}, w_{[1: 2]}, \mathbf{f}\right) \mathbb{1}\left\{\hat{w}_{1}=\check{w}_{1}=w_{1}, \hat{w}_{2}=\check{w}_{2}=w_{2}\right\},
$$


Using Equations (55) and (71) and Lemma 2, Equation (68) leads to

$$
\begin{aligned}
& \hat{P}\left(z^{n}, w_{[1: 2]}, \mathbf{f}, \hat{w}_{1}, \check{w}_{1}, \hat{w}_{2}, \check{w}_{2}\right) \\
& \approx p\left(z^{n}\right) p^{U}\left(w_{[1: 2]}, f_{[1: 2]}\right) p^{U}\left(f_{[1: 2]}^{\prime}, f_{[1: 2]}^{\prime \prime}\right) \mathbb{1}\left\{\hat{w}_{1}=\check{w}_{1}=w_{1}, \hat{w}_{2}=\check{w}_{2}=w_{2}\right\} .
\end{aligned}
$$

We now eliminate the shared randomness $\left(F_{[1: 2]}, F_{[1: 2]}^{\prime}, F_{[1: 2]}^{\prime \prime}\right)$ without affecting the secrecy and reliability requirements. By using Definition 3, Equation (72) ensures that there exists a fixed binning with corresponding pmf $p$ that, if used in place of the random coding strategy $P$ in (39), will induce the pmf $\hat{p}$ as follows:

$$
\begin{aligned}
& \hat{p}\left(z^{n}, w_{[1: 2]}, f_{[1: 2]}, f_{[1: 2]}^{\prime}, f_{[1: 2]}^{\prime \prime}, \hat{w}_{1}, \check{w}_{1}, \hat{w}_{2}, \check{w}_{2}\right) \\
& \approx p\left(z^{n}\right) p^{U}\left(w_{[1: 2]}, f_{[1: 2]}\right) p^{U}\left(f_{[1: 2]}^{\prime}, f_{[1: 2]}^{\prime \prime}\right) \mathbb{1}\left\{\hat{w}_{1}=\check{w}_{1}=w_{1}, \hat{w}_{2}=\check{w}_{2}=w_{2}\right\} .
\end{aligned}
$$

Now, using Lemma 2, Equation (67) shows that there exists an instance of $\left(f_{[1: 2]}, f_{[1: 2]}^{\prime}, f_{[1: 2]}^{\prime \prime}\right)$ such that:

$$
\begin{aligned}
& \hat{p}\left(z^{n}, w_{[1: 2]}, \hat{w}_{1}, \check{w}_{1}, \hat{w}_{2}, \check{w}_{2} \mid f_{[1: 2]}, f_{[1: 2]}^{\prime}, f_{[1: 2]}^{\prime \prime}\right) \\
& \approx p\left(z^{n}\right) p^{U}\left(w_{1}\right) p^{U}\left(w_{2}\right) \mathbb{1}\left\{\hat{w}_{1}=\check{w}_{1}=w_{1}, \hat{w}_{2}=\check{w}_{2}=w_{2}\right\} .
\end{aligned}
$$

This distribution satisfies the secrecy and reliability requirements as follows:

- Reliability: Using Lemma 2, Equation (66) leads to

$$
\hat{p}\left(w_{[1: 2]}, \hat{w}_{1,1}, \hat{w}_{1,2}, \hat{w}_{2,1}, \hat{w}_{2,2} \mid f_{[1: 2]}, f_{[1: 2]}^{\prime}, f_{[1: 2]}^{\prime \prime}\right) \approx \mathbb{1}\left\{\hat{w}_{1}=\check{w}_{1}=w_{1}, \hat{w}_{2}=\check{w}_{2}=w_{2}\right\}
$$

which is equivalent to:

$$
\hat{p}\left(\left\{\left(\hat{W}_{1}, \hat{W}_{2}\right) \neq\left(W_{1}, W_{2}\right)\right\} \cup\left\{\left(\check{W}_{1}, \check{W}_{2}\right) \neq\left(W_{1}, W_{2}\right)\right\} \mid f_{[1: 2]}, f_{[1: 2]}^{\prime}, f_{[1: 2]}^{\prime \prime}\right) \rightarrow 0 .
$$

- Security: Again, using Lemma 2, Equation (66)

$$
\hat{p}\left(z^{n}, w_{[1: 2]} \mid f_{[1: 2]}, f_{[1: 2]}^{\prime}, f_{[1: 2]}^{\prime \prime}\right) \approx p\left(z^{n}\right) p^{U}\left(w_{1}\right) p^{U}\left(w_{2}\right) .
$$

Finally, we identify $p\left(x_{1}^{n} \mid w_{1}, f_{1}, f_{[1: 2]}^{\prime}\right)$ and $p\left(x_{2}^{n} \mid w_{2}, f_{2}, f_{[1: 2]}^{\prime \prime}\right)$ (which is done by generating $u_{[0: 2]}$ and $v_{[0: 2]}$ first, respectively) as encoders and the Slepian-Wolf decoders as decoders for the channel coding problem. These encoders and decoders lead to reliable and secure encoders and decoders.

By applying a computer generated Fourier-Motzkin procedure to (40)-(51), (57), (58), and (62) the achievable rate region for the strong secrecy regime in Theorem 6 is obtained [32]. 
Remark 3. If we assume that (5), and therefore (6), holds, the inequalities (45) for $j=2$, (46) for $j=1$, and (47)-(51) will be redundant and by applying the Fourier-Motzkin procedure [32], [33] to (40)-(44), (45) for $j=1$, (46) for $j=2$, (57), (58), and (62) the region in Theorem 1 ] over the distribution (33) will be achieved. This shows that the region derived by OSRB is a superset of the region derived in the weak secrecy regime.

Remark 4. The random distributions $P\left(u_{0}^{n}, v_{0}^{n} \mid w_{[1: 2]}, f_{[1: 2]}\right)$ and $P\left(u_{[1: 2]}^{n}, v_{[1: 2]}^{n} \mid u_{0}^{n}, v_{0}^{n}, f_{[1: 2]}^{\prime}, f_{[1: 2]}^{\prime \prime}\right)$ factorize as $P\left(u_{0}^{n} \mid w_{1}, f_{1}\right) P\left(v_{0}^{n} \mid w_{2}, f_{2}\right)$ and $P\left(u_{[1: 2]}^{n} \mid u_{0}^{n}, f_{[1: 2]}^{\prime}\right) P\left(v_{[1: 2]}^{n} \mid v_{0}^{n}, f_{[1: 2]}^{\prime \prime}\right)$, respectively, which means that Encoders 1 and 2 are not using the common randomness and the message available at the other encoder to generate the common and private random variables. The common randomness $\left(F_{1}, F_{[1: 2]}^{\prime}\right)$ represents the realization of Encoder 1 's codebook and $\left(F_{2}, F_{[1: 2]}^{\prime \prime}\right)$ represents the realization of Encoder 2's codebook, which is available at all terminals, but the codebook at one encoder does not depend on the codebook of the other encoder.

Remark 5. The achievable region described in the proof of Theorem 6 was without time sharing, i.e., $Q=\emptyset$. One can incorporate this into the proof by generating i.i.d. copies of $Q$, and sharing it among all terminals and conditioning everything on it.

\section{APPENDIX A}

\section{ProOF OF LEMMA 1}

Let $N\left(Q^{n}, U_{0}^{n}, V_{0}^{n}, Z^{n}\right)=\mid\left\{(k, \ell) \in \llbracket 1,2^{n S} \rrbracket \times \llbracket 1,2^{n T} \rrbracket:\left(Q^{n}, U_{0}^{n}, V_{0}^{n}, U_{1}^{n}(k), V_{1}^{n}(\ell), Z^{n}\right) \in\right.$ $\left.\mathcal{T}_{\epsilon}^{(n)}\right\} \mid$. Next, let's define the following error events.

Let $E_{1}\left(Q^{n}, U_{0}^{n}, V_{0}^{n}, Z^{n}\right)=1$ if $N\left(Q^{n}, U_{0}^{n}, V_{0}^{n}, Z^{n}\right) \geq\left(1+\delta_{1}(\epsilon)\right) 2^{n\left(S+T-\mathbb{I}\left(U_{1}, V_{1} ; Z \mid Q, U_{0}, V_{0}\right)+\delta(\epsilon)\right)}$ and $E_{1}=0$ otherwise.

Let $E=0$ if $\left(Q^{n}, U_{0}^{n}, V_{0}^{n}, U_{1}^{n}(K), V_{1}^{n}(L), Z^{n}\right) \in \mathcal{T}_{\epsilon}^{(n)}$ and $E_{1}\left(Q^{n}, U_{0}^{n}, V_{0}^{n}, Z^{n}, K, L\right)=0$, and $E=1$ otherwise.

We now show that if $S \geq \mathbb{I}\left(U_{1} ; Z \mid Q, U_{0}, V_{0}\right)+\delta(\epsilon), T \geq \mathbb{I}\left(V_{1} ; Z \mid Q, U_{0}, V_{0}\right)+\delta(\epsilon)$, and $S+T \geq \mathbb{I}\left(U_{1}, V_{1} ; Z \mid Q, U_{0}, V_{0}\right)+\delta(\epsilon)$, then $\mathbb{P}(E=1) \rightarrow 0$ as $n \rightarrow \infty$.

By the union bound we have

$$
\mathbb{P}(E=1) \leq \mathbb{P}\left(\left(Q^{n}, U_{0}^{n}, V_{0}^{n}, U_{1}^{n}(K), V_{1}^{n}(L), Z^{n}\right) \notin \mathcal{T}_{\epsilon}^{(n)}\right)+\mathbb{P}\left(E_{1}\left(Q^{n}, U_{0}^{n}, V_{0}^{n}, Z^{n}, K, L\right)=1\right)
$$

The first term tends to zero by the main assumption of the Lemma. 
We then partition the event $\left\{E_{1}=1\right\}$ based on the composition of the typical sequences $\left(Q^{n}, U_{0}^{n}, V_{0}^{n}, U_{1}^{n}(k), V_{1}^{n}(\ell), Z^{n}\right) \in \mathcal{T}_{\epsilon}^{(n)}:$

- When all such typical sequences share the same $U_{1}^{n}(k)$, i.e., correspond to a single $k$.

- When all such typical sequences share the same $V_{1}^{n}(\ell)$, i.e., correspond to a single $\ell$.

- Neither of the above

As usual, each of the three partitioned $E_{1}$ events gives rise to one rate constraint. We discuss the first in detail; the remaining two follow similarly. Define $A\left(Q^{n}, U_{0}^{n}, V_{0}^{n}, z^{n}\right)$ as the event $\left\{E_{1}\left(Q^{n}, U_{0}^{n}, V_{0}^{n}, Z^{n}\right)=1\right\} \cap\left\{Z^{n}=z^{n}\right\}$,

$$
\begin{aligned}
& \mathbb{P}\left(E_{1}\left(Q^{n}, U_{0}^{n}, V_{0}^{n}, Z^{n}\right)=1\right) \\
& =\sum_{\substack{\left(q^{n}, u_{0}^{n}, v_{0}^{n}\right) \in \mathcal{T}_{\epsilon}^{(n)} \\
=}}\left[p\left(q^{n}\right) p\left(u_{0}^{n} \mid q^{n}\right) p\left(v_{0}^{n} \mid q^{n}\right) \times \mathbb{P}\left(\left(E_{1}\left(Q^{n}, U_{0}^{n}, V_{0}^{n}, Z^{n}\right)=1\right) \mid Q^{n}=q^{n}, U_{0}^{n}=u_{0}^{n}, V_{0}^{n}=v_{0}^{n}\right)\right] \\
& \sum_{\substack{\left(q^{n}, u_{0}^{n}, v_{0}^{n}\right) \in \mathcal{T}_{\epsilon}^{(n)}\left(Q, U_{0}, V_{0}\right) \\
z^{n} \in \mathcal{T}_{\epsilon}^{(n)}\left(Z \mid Q, U_{0}, V_{0}\right)}} p\left(q^{n}\right) p\left(u_{0}^{n} \mid q^{n}\right) p\left(v_{0}^{n} \mid q^{n}\right) \mathbb{P}\left(A\left(q^{n}, u_{0}^{n}, v_{0}^{n}, z^{n}\right) \mid Q^{n}=q^{n}, U_{0}^{n}=u_{0}^{n}, V_{0}^{n}=v_{0}^{n}\right) \\
& \quad \sum_{\substack{\left.\left(q^{n}, u_{0}^{n}, v_{0}^{n}\right) \in \mathcal{T}_{\epsilon}^{(n)}\left(Q, U_{0}, V_{0}\right) \\
\mid Q^{n}=q^{n}, U_{0}^{n}=u_{0}^{n}, V_{0}^{n}=v_{0}^{n}\right) .}} p\left(q^{n}\right) p\left(u_{0}^{n} \mid q^{n}\right) p\left(v_{0}^{n} \mid q^{n}\right) \sum_{z^{n} \in \mathcal{T}_{\epsilon}^{(n)}\left(Z \mid Q, U_{0}, V_{0}\right)} \mathbb{P}\left(\left(E_{1}\left(q^{n}, u_{0}^{n}, v_{0}^{n}, z^{n}\right)=1\right)\right.
\end{aligned}
$$

Then,

$$
\begin{aligned}
& \mathbb{P}\left(E_{1}\left(q^{n}, u_{0}^{n}, v_{0}^{n}, z^{n}\right)=1 \mid Q^{n}=q^{n}, U_{0}^{n}=u_{0}^{n}, V_{0}^{n}=v_{0}^{n}\right)= \\
& \mathbb{P}\left(N\left(q^{n}, u_{0}^{n}, v_{0}^{n}, z^{n}\right) \geq\left(1+\delta_{1}(\epsilon)\right) 2^{n\left(T-\mathbb{I}\left(V_{1} ; Z \mid Q, U_{0}, V_{0}\right)+\delta(\epsilon)\right)}\right) .
\end{aligned}
$$

Define $X_{\ell}=1$ if $\left(q^{n}, u_{0}^{n}, v_{0}^{n}, V_{1}^{n}(\ell), z^{n}\right) \in \mathcal{T}_{\epsilon}^{(n)}$ and 0 otherwise. Here, $X_{\ell}, \ell \in \llbracket 1,2^{n T} \rrbracket$, are i.i.d. Bernoulli- $\alpha$ random variables, where

$$
2^{-n\left(\mathbb{I}\left(V_{1} ; Z \mid Q, U_{0}, V_{0}\right)+\delta(\epsilon)\right)} \leq \alpha \leq 2^{-n\left(\mathbb{I}\left(V_{1} ; Z \mid Q, U_{0}, V_{0}\right)-\delta(\epsilon)\right)}
$$

Then

$$
\begin{aligned}
& \mathbb{P}\left(N\left(q^{n}, u_{0}^{n}, v_{0}^{n}, z^{n}\right) \geq\left(1+\delta_{1}(\epsilon)\right) 2^{n\left(T-\mathbb{I}\left(V_{1} ; Z \mid Q, U_{0}, V_{0}\right)+\delta(\epsilon)\right)} \mid Q^{n}=q^{n}, U_{0}^{n}=u_{0}^{n}, V_{0}^{n}=v_{0}^{n}\right) \\
& \leq \mathbb{P}\left(\sum_{\ell=1}^{2^{n T}} X_{\ell} \geq\left(1+\delta_{1}(\epsilon)\right) 2^{n T} \alpha \mid Q^{n}=q^{n}, U_{0}^{n}=u_{0}^{n}, V_{0}^{n}=v_{0}^{n}\right) .
\end{aligned}
$$


Applying the Chernoff Bound (e.g., see [34, Appendix B]), leads to

$$
\begin{aligned}
& \mathbb{P}\left(\sum_{\ell=1}^{2^{n T}} X_{\ell} \geq\left(1+\delta_{1}(\epsilon)\right) 2^{n T} \alpha \mid Q^{n}=q^{n}, U_{0}^{n}=u_{0}^{n}, V_{0}^{n}=v_{0}^{n}\right) \\
& \leq \exp \left(-2^{n T} \alpha \delta_{1}^{2}(\epsilon) / 4\right) \\
& \leq \exp \left(-2^{n\left(T-\mathbb{I}\left(V_{1} ; Z \mid Q, U_{0}, V_{0}\right)-\delta(\epsilon)\right)} \delta_{1}^{2}(\epsilon) / 4\right) .
\end{aligned}
$$

Therefore,

$$
\begin{aligned}
& \mathbb{P}\left(E_{1}\left(Q^{n}, U_{0}^{n}, V_{0}^{n}, Z^{n}\right)=1\right) \\
& \leq \sum_{\left(q^{n}, u_{0}^{n}, v_{0}^{n}\right) \in \mathcal{T}_{\epsilon}^{(n)}} p\left(q^{n}\right) p\left(u_{0}^{n} \mid q^{n}\right) p\left(v_{0}^{n} \mid q^{n}\right) \sum_{z^{n} \in \mathcal{T}_{\epsilon}^{(n)}\left(Z \mid Q, U_{0}, V_{0}\right)} \exp \left(-2^{n\left(T-\mathbb{I}\left(V_{1} ; Z \mid Q, U_{0}, V_{0}\right)-\delta(\epsilon)\right)} \delta_{1}^{2}(\epsilon) / 4\right) \\
& \leq 2^{n \log |\mathcal{Z}|} \exp \left(-2^{n\left(T-\mathbb{I}\left(V_{1} ; Z \mid Q, U_{0}, V_{0}\right)-\delta(\epsilon)\right)} \delta_{1}^{2}(\epsilon) / 4\right)
\end{aligned}
$$

which tends to zero as $n \rightarrow \infty$ if $T \geq \mathbb{I}\left(V_{1} ; Z \mid Q, U_{0}, V_{0}\right)+\delta(\epsilon)$.

In a similar manner, the bounding of error probability for the second and third partition of $E_{1}$ (please see above) will give rise to the rate constraints $S \geq \mathbb{I}\left(U_{1} ; Z \mid Q, U_{0}, V_{0}\right)+\delta(\epsilon)$, and $S+T \geq \mathbb{I}\left(U_{1}, V_{1} ; Z \mid Q, U_{0}, V_{0}\right)+\delta(\epsilon)$, respectively. Details are ommited for brevity.

Finally, we bound $\mathbb{H}\left(L, K \mid Q^{n}, U_{0}^{n}, V_{0}^{n}, Z^{n}, \mathcal{C}\right)$ as follows:

$$
\begin{aligned}
& \mathbb{H}\left(L, K, E \mid Q^{n}, U_{0}^{n}, V_{0}^{n}, Z^{n}, C\right) \\
& \leq 1+\mathbb{P}(E=1) \mathbb{H}\left(L, K \mid E=1, Q^{n}, U_{0}^{n}, V_{0}^{n}, Z^{n}, C\right)+\mathbb{P}(E=0) \mathbb{H}\left(L, K \mid E=0, Q^{n}, U_{0}^{n}, V_{0}^{n}, Z^{n}, C\right) \\
& \leq 1+\mathbb{P}(E=1) n(S+T)+\log \left(\left(1+\delta_{1}(\epsilon)\right) 2^{n\left(S+T-\mathbb{I}\left(U_{1}, V_{1} ; Z \mid Q, U_{0}, V_{0}\right)+\delta(\epsilon)\right)}\right) \\
& \leq n\left(S+T-\mathbb{I}\left(U_{1}, V_{1} ; Z \mid Q, U_{0}, V_{0}\right)+\delta_{2}(\epsilon)\right)
\end{aligned}
$$

\section{APPENDIX B}

\section{ACHIEvable RATE REgion FOR MAC-WTC Under RANDOMnESS CONSTRAint}

It is well-known that a stochastic encoding is required to avoid leaking information about the transmitted confidential messages to an eavesdropper. Here, a new achievability technique for characterizing the trade-off between the rate of the random number to realize the stochastic encoding and the communication rates in multiple access wiretap channel, by employing a variation of superposition coding, is presented.

Consider a MAC-WTC $\left(\mathcal{X}_{1}, \mathcal{X}_{2}, p\left(y, z \mid x_{1}, x_{2}\right), \mathcal{Y}, \mathcal{Z}\right)$, in which $\mathcal{X}_{1}, \mathcal{X}_{2}$ are finite input alphabets and $\mathcal{Y}$ and $\mathcal{Z}$ are finite output alphabets at the legitimate receiver and the eavesdropper, 


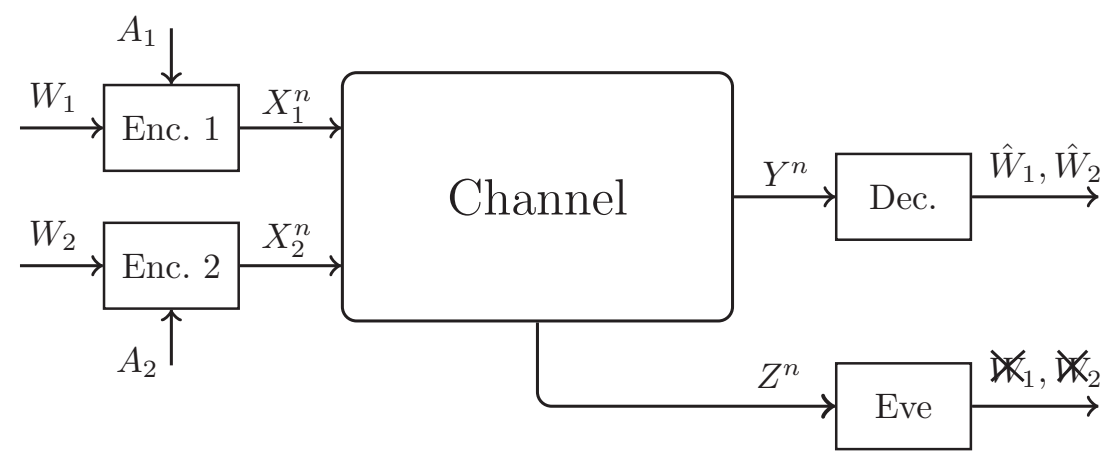

Fig. 7. Multiple access wiretap channel

respectively (as depicted in Fig. 7). In this problem, each transmitter sends a confidential message which is supposed to be decoded by the legitimate receiver and must be kept secret from the eavesdropper. Furthermore, for stochastic encoding, Encoder 1 and Encoder 2 are allowed to use a limited amount of randomness. Thus, we are interested in the trade-off between the rate of randomness, and the rates of confidential messages.

Definition 5. $A\left(M_{1, n}, M_{2, n}, n\right)$ code for the considered model (Fig. 7) consists of the following:

i) Two message sets $\mathcal{W}_{i}=\llbracket 1, M_{i, n} \rrbracket, i=1,2$, from which independent messages $W_{1}$ and $W_{2}$ are drawn uniformly distributed over their respective sets. Also, Two dummy message sets $\mathcal{A}_{i}=\llbracket 1, M_{i, n}^{\prime} \rrbracket, i=1,2$, from which independent dummy messages $A_{1}$ and $A_{2}$ are drawn uniformly distributed over their respective sets.

ii) Deterministic encoders $f_{i, n}, i=1,2$, are defined by function $f_{i, n}: \mathcal{W}_{i} \times \mathcal{A}_{i} \rightarrow \mathcal{X}_{i}^{n}$.

iii) A decoding function $\phi: \mathcal{Y}^{n} \rightarrow \mathcal{W}_{1} \times \mathcal{W}_{2}$ that assigns $\left(\hat{w}_{1}, \hat{w}_{2}\right) \in \llbracket 1, M_{1, n} \rrbracket \times \llbracket 1, M_{2, n} \rrbracket$ to received sequence $y^{n}$.

The probability of error is given by:

$$
P_{e} \triangleq \mathbb{P}\left(\left\{\left(\hat{W}_{1}, \hat{W}_{2}\right) \neq\left(w_{1}, w_{2}\right)\right\}\right)
$$

Definition 6 ( [28]). A quadruple $\left(R_{1}, R_{d_{1}}, R_{2}, R_{d_{2}}\right)$ is achievable under weak secrecy if there exists a sequence of $\left(M_{1, n}, M_{2, n}, M_{1, n}^{\prime}, M_{2, n}^{\prime}, n\right)$ codes with $M_{1, n} \geq 2^{n R_{1}}, M_{2, n} \geq 2^{n R_{2}}, M_{1, n}^{\prime} \leq$ $2^{n R_{d_{1}}}, M_{2, n}^{\prime} \leq 2^{n R_{d_{2}}}$, so that $P_{e} \underset{n \rightarrow \infty}{\longrightarrow} 0$ and

$$
\frac{1}{n} \mathbb{I}\left(W_{1}, W_{2} ; Z^{n}\right) \underset{n \rightarrow \infty}{\longrightarrow} 0 .
$$


Theorem 7. An inner bound on the secrecy capacity region of the multiple access wiretap channel is given by the set of non-negative quadruple $\left(R_{1}, R_{d_{1}}, R_{2}, R_{d_{2}}\right)$ such that

$$
\begin{aligned}
R_{1} & \leq \mathbb{I}(U ; Y \mid Q, V)-\mathbb{I}(U ; Z \mid Q), \\
R_{2} & \leq \mathbb{I}(V ; Y \mid Q, U)-\mathbb{I}(V ; Z \mid Q), \\
R_{1}+R_{2} & \leq \mathbb{I}(U, V ; Y \mid Q)-\mathbb{I}(U, V ; Z \mid Q), \\
R_{d_{1}} & \geq \mathbb{I}(U ; Z \mid Q)+\mathbb{I}\left(X_{1} ; Z \mid Q, U, V\right), \\
R_{d_{2}} & \geq \mathbb{I}(V ; Z \mid Q)+\mathbb{I}\left(X_{2} ; Z \mid Q, U, V\right), \\
R_{d_{1}}+R_{d_{2}} & \geq \mathbb{I}(U, V ; Z \mid Q)+\mathbb{I}\left(X_{1}, X_{2} ; Z \mid Q, U, V\right),
\end{aligned}
$$

for some

$$
p(q) p(u \mid q) p(v \mid q) p\left(x_{1} \mid u\right) p\left(x_{2} \mid v\right) p\left(y, z \mid x_{1}, x_{2}\right) .
$$

Remark 6. By setting $U=X_{1}, V=X_{2}$, and by taking sufficiently large $R_{d_{1}}$ and $R_{d_{2}}$, the result in Theorem 7 reduces to the achievable rate region of multiple access wiretap channel without common message [18]-[20].

Remark 7. By setting $X_{2}=\emptyset$ and $V=\emptyset\left(\right.$ or $X_{1}=\emptyset$ and $\left.U=\emptyset\right)$, the result in Theorem 7 reduces to the capacity rate region of broadcast channel with confidential messages under randomness constraint in [12 Corollary 11].

Proof. Rate Splitting: Divide the dummy message $A_{1}$ into independent dummy messages $A_{1,1} \in$ $\llbracket 1,2^{n R_{1,1}} \rrbracket$ and $A_{1,2} \in \llbracket 1,2^{n R_{1,2}} \rrbracket$. Also, divide the dummy message $A_{2}$ into independent dummy messages $A_{2,1} \in \llbracket 1,2^{n R_{2,1}} \rrbracket$ and $A_{2,2} \in \llbracket 1,2^{n R_{2,2}} \rrbracket$. Therefore, $R_{d_{1}}=R_{1,1}+R_{1,2}$ and $R_{d_{2}}=$ $R_{2,1}+R_{2,2}$.

Codebook Generation: Fix $p(q), p(u \mid q), p(v \mid q), p\left(x_{1} \mid u\right), p\left(x_{2} \mid v\right)$, and $\epsilon>0$. Randomly and independently generate a typical sequence $q^{n}$ according to $p\left(q^{n}\right)=\prod_{i=1}^{n} p\left(q_{i}\right)$. We suppose that all the terminals know $q^{n}$.

i) Generate $2^{n\left(R_{1}+R_{1,1}\right)}$ sequences according to $\prod_{i=1}^{n} p_{U \mid Q}\left(u_{i} \mid q_{i}\right)$. Then, randomly bin these $2^{n\left(R_{1}+R_{1,1}\right)}$ sequences into $2^{n R_{1}}$ bins. We index these sequences as $u^{n}\left(w_{1}, a_{1,1}\right)$. For each $\left(w_{1}, a_{1,1}\right)$, generate $2^{n R_{1,2}}$ codewords $x_{1}^{n}\left(w_{1}, a_{1,1}, a_{1,2}\right)$ each according to $\prod_{i=1}^{n} p_{X_{1} \mid U}\left(x_{1, i} \mid u_{i}\right)$. 
ii) Generate $2^{n\left(R_{2}+R_{2,1}\right)}$ sequences according to $\prod_{i=1}^{n} p_{V \mid Q}\left(v_{i} \mid q_{i}\right)$. Then, randomly bin these $2^{n\left(R_{2}+R_{2,1}\right)}$ sequences into $2^{n R_{2}}$ bins. We index these sequences as $v^{n}\left(w_{2}, a_{2,1}\right)$. For each $\left(w_{2}, a_{2,1}\right)$, generate $2^{n R_{2,2}}$ codewords $x_{1}^{n}\left(w_{2}, a_{2,1}, a_{2,2}\right)$ each according to $\prod_{i=1}^{n} p_{X_{2} \mid V}\left(x_{2, i} \mid v_{i}\right)$.

Encoding: To send the message $w_{1}$, the Encoder 1 splits $a_{1}$ into $\left(a_{1,1}, a_{1,2}\right)$, and chooses $u^{n}\left(w_{1}, a_{1,1}\right)$. Then it chooses codeword $x_{1}^{n}\left(w_{1}, a_{1,1}, a_{1,2}\right)$ and send it over the channel.

To send the message $w_{2}$, the Encoder 2 splits $a_{2}$ into $\left(a_{2,1}, a_{2,2}\right)$, and chooses $v^{n}\left(w_{2}, a_{2,1}\right)$. Then it chooses codeword $x_{2}^{n}\left(w_{2}, a_{2,1}, a_{2,2}\right)$ and send it over the channel.

Decoding and Error Probability Analysis:

- Decoder decodes $\left(w_{1}, w_{2}\right)$ by finding a unique pair $\left(w_{1}, w_{2}\right)$ such that $\left(q^{n}, u^{n}\left(w_{1}, a_{1,1}\right), v^{n}\left(w_{2}, a_{2,1}\right), y^{n}\right) \in \mathcal{T}_{\epsilon}^{(n)}\left(p_{U, V, Y}\right)$ for some $\left(a_{1,1}, a_{2,1}\right)$. The probability of error for Receiver goes to zero as $n \rightarrow \infty$ if we choose [34]

$$
\begin{aligned}
& R_{1}+R_{1,1} \leq \mathbb{I}(U ; Y \mid Q, V)-\epsilon, \\
& R_{2}+R_{2,1} \leq \mathbb{I}(V ; Y \mid Q, U)-\epsilon, \\
& R_{1}+R_{1,1}+R_{2}+R_{2,1} \leq \mathbb{I}(U, V ; Y \mid Q)-\epsilon .
\end{aligned}
$$

Equivocation Calculation: We analyze mutual information between $\left(W_{1}, W_{2}\right)$ and $Z^{n}$, averaged over all random codebooks

$$
\begin{aligned}
& \mathbb{I}\left(W_{1}, W_{2} ; Z^{n} \mid Q^{n}, \mathcal{C}\right) \\
= & \mathbb{I}\left(W_{1}, W_{2}, A_{1,1}, A_{1,2}, A_{2,1}, A_{2,2} ; Z^{n} \mid Q^{n}, \mathcal{C}\right)-\mathbb{I}\left(A_{1,1}, A_{1,2}, A_{2,1}, A_{2,2} ; Z^{n} \mid W_{1}, W_{2}, Q^{n}, \mathcal{C}\right) \\
\stackrel{(a)}{=} & \mathbb{I}\left(W_{1}, W_{2}, A_{1,1}, A_{1,2}, A_{2,1}, A_{2,2}, X_{1}^{n}, X_{2}^{n} ; Z^{n} \mid Q^{n}, \mathcal{C}\right)-\mathbb{I}\left(A_{1,1}, A_{1,2}, A_{2,1}, A_{2,2} ; Z^{n} \mid W_{1}, W_{2}, Q^{n}, \mathcal{C}\right) \\
\stackrel{(b)}{=} & \mathbb{I}\left(X_{1}^{n}, X_{2}^{n} ; Z^{n} \mid Q^{n}, \mathcal{C}\right)-\mathbb{I}\left(A_{1,1}, A_{1,2}, A_{2,1}, A_{2,2} ; Z^{n} \mid W_{1}, W_{2}, Q^{n}, \mathcal{C}\right) \\
= & \mathbb{I}\left(X_{1}^{n}, X_{2}^{n} ; Z^{n} \mid Q^{n}, \mathcal{C}\right)-\mathbb{I}\left(A_{1,1}, A_{2,1} ; Z^{n} \mid W_{1}, W_{2}, Q^{n}, \mathcal{C}\right) \\
& \quad-\mathbb{I}\left(A_{1,2}, A_{2,2} ; Z^{n} \mid W_{1}, W_{2}, A_{1,1}, A_{1,2}, Q^{n}, \mathcal{C}\right) \\
= & \mathbb{I}\left(X_{1}^{n}, X_{2}^{n} ; Z^{n} \mid Q^{n}, \mathcal{C}\right)-\mathbb{H}\left(A_{1,1}, A_{2,1} \mid W_{1}, W_{2}, Q^{n}, \mathcal{C}\right)+\mathbb{H}\left(A_{1,1}, A_{2,1} \mid W_{1}, W_{2}, Z^{n}, Q^{n}, \mathcal{C}\right) \\
& -\mathbb{H}\left(A_{1,2}, A_{2,2} \mid W_{1}, W_{2}, A_{1,1}, A_{2,1}, Q^{n}, \mathcal{C}\right)+\mathbb{H}\left(A_{1,2}, A_{2,2} \mid W_{1}, W_{2}, A_{1,1}, A_{2,1}, Z^{n}, Q^{n}, \mathcal{C}\right)
\end{aligned}
$$

where $(a)$ is due to $X_{1}^{n}$ and $X_{2}^{n}$ are deterministic functions of $\left(W_{1}, A_{1,1}, A_{1,2}\right)$ and $\left(W_{2}, A_{2,1}, A_{2,2}\right)$, respectively. Also, $(b)$ is due to the fact that, given $X_{1}^{n}$ and $X_{2}^{n}$, the indices $W_{1}, W_{2}, A_{1,1}, A_{1,2}, A_{2,1}$, and $A_{2,2}$ are uniquely determined. 
The first term in (94) is bounded as:

$$
\mathbb{I}\left(X_{1}^{n}, X_{2}^{n} ; Z^{n} \mid Q^{n}, \mathcal{C}\right) \leq n \mathbb{I}\left(X_{1}, X_{2} ; Z \mid Q\right)+n \epsilon
$$

where $\epsilon \underset{n \rightarrow \infty}{\longrightarrow} 0$ similar to [34].

For the second term in (94) we have

$$
\mathbb{H}\left(A_{1,1}, A_{2,1} \mid W_{1}, W_{2}, Q^{n}, \mathcal{C}\right)=n\left(R_{1,1}+R_{2,1}\right) .
$$

For the third term, substituting $U_{0} \leftarrow Q, V_{0} \leftarrow Q, U_{1} \leftarrow U$, and $V_{1} \leftarrow V$ in Lemma 1 result that if $\mathbb{P}\left(\left(Q^{n}, U^{n}\left(W_{1}, A_{1,1}\right), V^{n}\left(W_{2}, A_{2,1}\right), Z^{n}\right) \in \mathcal{T}_{\epsilon}^{(n)}\right) \underset{n \rightarrow \infty}{\longrightarrow} 1$ and

$$
\begin{aligned}
R_{1,1} & \geq \mathbb{I}(U ; Z \mid Q)+\epsilon, \\
R_{2,1} & \geq \mathbb{I}(V ; Z \mid Q)+\epsilon, \\
R_{1,1}+R_{2,1} & \geq \mathbb{I}(U, V ; Z \mid Q)+\epsilon .
\end{aligned}
$$

Then,

$$
\mathbb{H}\left(A_{1,1}, A_{2,1} \mid W_{1}, W_{2}, Z^{n}, Q^{n}, \mathcal{C}\right) \leq n\left(R_{1,1}+R_{2,1}-\mathbb{I}(U, V ; Z \mid Q)+\epsilon\right)
$$

Here, this condition holds because

$$
\mathbb{P}\left(\left(Q^{n}, U^{n}\left(W_{1}, A_{1,1}\right), X_{1}^{n}\left(W_{1}, A_{1,1}, A_{1,2}\right), V^{n}\left(W_{2}, A_{2,1}\right), X_{2}^{n}\left(W_{2}, A_{2,1}, A_{2,2}\right), Z^{n}\right) \in \mathcal{T}_{\epsilon}^{(n)}\right) \underset{n \rightarrow \infty}{\longrightarrow} 1
$$

Now, we bound the fourth term in (94),

$$
\mathbb{H}\left(A_{1,2}, A_{2,2} \mid W_{1}, W_{2}, A_{1,1}, A_{2,1}, Q^{n}, \mathcal{C}\right)=n\left(R_{1,2}+R_{2,2}\right)
$$

Now, we bound the last term in (94) by applying Lemma 1 ,

$$
\mathbb{H}\left(A_{1,2}, A_{2,2} \mid W_{1}, W_{2}, A_{1,1}, A_{2,1}, Z^{n}, Q^{n}, \mathcal{C}\right) \leq n\left(R_{1,2}+R_{2,2}-\mathbb{I}\left(X_{1}, X_{2} ; Z \mid Q, U, V\right)+\epsilon\right),
$$

if (101) holds and

$$
\begin{gathered}
R_{1,2} \geq \mathbb{I}\left(X_{1} ; Z \mid Q, U, V\right)+\epsilon, \\
R_{2,2} \geq \mathbb{I}\left(X_{2} ; Z \mid Q, U, V\right)+\epsilon, \\
R_{1,2}+R_{2,2} \geq \mathbb{I}\left(X_{1}, X_{2} ; Z \mid Q, U, V\right)+\epsilon .
\end{gathered}
$$


Substituting (95), (96), (100), (102), and (103) into (94) yields

$$
\begin{aligned}
& \mathbb{I}\left(W_{1}, W_{2} ; Z^{n} \mid Q^{n}, \mathcal{C}\right) \leq n \mathbb{I}\left(X_{1}, X_{2} ; Z \mid Q\right)-n\left(R_{1,1}+R_{2,1}\right) \\
& +n\left(R_{1,1}+R_{2,1}-\mathbb{I}(U, V ; Z \mid Q)+\epsilon\right)-n\left(R_{1,2}+R_{2,2}\right) \\
& +n\left(R_{1,2}+R_{2,2}-\mathbb{I}\left(X_{1}, X_{2} ; Z \mid Q, U, V\right)+\epsilon\right) .
\end{aligned}
$$

Therefore $\mathbb{I}\left(W_{1}, W_{2} ; Z^{n} \mid Q^{n}, \mathcal{C}\right) \leq 2 n \epsilon$. By applying the Fourier-Motzkin procedure [32] to (91)(93), (97)-(99), (104)-(106), $R_{d_{1}}=R_{1,1}+R_{1,2}$, and $R_{d_{2}}=R_{2,1}+R_{2,2}$ we obtain the region in Theorem 7 .

\section{APPENDIX C}

\section{Proof of TheOREM 1}

The coding scheme is based on superposition coding, Wyner's random binning [35], Marton coding, and applying indirect decoding [7].

The random code generation is as follows:

Fix $p(q), p\left(u_{0} \mid q\right), p\left(u_{1}, u_{2} \mid u_{0}\right), p\left(v_{0} \mid q\right), p\left(v_{1}, v_{2} \mid v_{0}\right), p\left(x_{1} \mid u_{0}, u_{1}, u_{2}\right), p\left(x_{2} \mid v_{0}, v_{1}, v_{2}\right), \epsilon_{1}<$ $\min \left\{\epsilon^{\prime}, \epsilon^{\prime \prime}\right\}$, and $\epsilon_{2}<\min \left\{\epsilon^{\prime}, \epsilon^{\prime \prime}\right\}$.

Codebook Generation: Randomly and independently generate a typical sequence $q^{n}$ according to $p\left(q^{n}\right)=\prod_{i=1}^{n} p\left(q_{i}\right)$. We suppose that all the terminals know $q^{n}$.

i) Generate $2^{n \tilde{R}_{1}}$ codewords $u_{0}^{n}\left(\ell_{0}\right)$ each according to $\prod_{i=1}^{n} p_{U_{0} \mid Q}\left(u_{0, i} \mid q_{i}\right)$. Then, randomly bin the $2^{n \tilde{R}_{1}}$ codewords into $2^{n R_{1}}$ bins, $\mathcal{B}\left(w_{1}\right), w_{1} \in \llbracket 1,2^{n R_{1}} \rrbracket$. For each $\ell_{0}$, generate $2^{n \rho_{1}}$ codewords $u_{1}^{n}\left(\ell_{0}, t_{1}\right)$ each according to $\prod_{i=1}^{n} p_{U_{1} \mid U_{0}}\left(u_{1, i} \mid u_{0, i}\right)$. Then, randomly bin the $2^{n \rho_{1}}$ codewords into $2^{n \rho_{1}^{\prime}}$ bins, $\mathcal{B}\left(\ell_{0}, \ell_{1}\right), \ell_{1} \in \llbracket 1,2^{n \rho_{1}^{\prime}} \rrbracket$. Similarly, for each $\ell_{0}$, generate $2^{n \tilde{\rho}_{1}}$ codewords $u_{2}^{n}\left(\ell_{0}, t_{2}\right)$ each according to $\prod_{i=1}^{n} p_{U_{2} \mid U_{0}}\left(u_{2, i} \mid u_{0, i}\right)$. Then, randomly bin the $2^{n \tilde{\rho}_{1}}$ codewords into $2^{n \tilde{\rho}_{1}^{\prime}}$ bins, $\mathcal{B}\left(\ell_{0}, \ell_{2}\right), \ell_{2} \in \llbracket 1,2^{n \tilde{\rho}_{1}^{\prime}} \rrbracket$.

ii) Similarly, generate $2^{n \tilde{R}_{2}}$ codewords $v_{0}^{n}\left(\ell_{0}^{\prime}\right)$ each according to $\prod_{i=1}^{n} p_{V_{0} \mid Q}\left(v_{0, i} \mid q_{i}\right)$. Then, randomly bin the $2^{n \tilde{R}_{2}}$ codewords into $2^{n R_{2}}$ bins, $\mathcal{B}\left(w_{2}\right), w_{2} \in \llbracket 1,2^{n R_{2}} \rrbracket$. For each $\ell_{0}^{\prime}$, generate $2^{n \rho_{2}}$ codewords $v_{1}^{n}\left(\ell_{0}^{\prime}, s_{1}\right)$ each according to $\prod_{i=1}^{n} p_{V_{1} \mid V_{0}}\left(v_{1, i} \mid v_{0, i}\right)$. Then, randomly bin the $2^{n \rho_{2}}$ codewords into $2^{n \rho_{2}^{\prime}}$ bins, $\mathcal{B}\left(\ell_{0}^{\prime}, \ell_{1}^{\prime}\right), \ell_{1}^{\prime} \in \llbracket 1,2^{n \rho_{2}^{\prime}} \rrbracket$. Similarly, for each $\ell_{0}^{\prime}$, generate $2^{n \tilde{\rho}_{2}}$ codewords $v_{2}^{n}\left(\ell_{0}^{\prime}, s_{2}\right)$ each according to $\prod_{i=1}^{n} p_{V_{2} \mid V_{0}}\left(v_{2, i} \mid v_{0, i}\right)$. Then, randomly bin the $2^{n \tilde{\rho}_{2}}$ codewords into $2^{n \tilde{\rho}_{2}^{\prime}}$ bins, $\mathcal{B}\left(\ell_{0}^{\prime}, \ell_{2}^{\prime}\right), \ell_{2}^{\prime} \in \llbracket 1,2^{n \tilde{\rho}_{2}^{\prime}} \rrbracket$.

Encoding: To send the message $w_{1}$, the encoder $f_{1}$ first uniformly chooses index $L_{0} \in \mathcal{B}\left(w_{1}\right)$. Then, it uniformly chooses a pair of indices $\left(L_{1}, L_{2}\right)$ and selects a jointly typical sequence pair 
$\left(u_{1}^{n}\left(L_{0}, t_{1}\left(L_{0}, L_{1}\right)\right), u_{2}^{n}\left(L_{0}, t_{2}\left(L_{0}, L_{1}\right)\right)\right) \in \mathcal{T}_{\epsilon 1}^{(n)}\left(U_{1}, U_{2} \mid U_{0}\right)$ in the product bin. If the encoder $f_{1}$ finds more than one such pair, then it chooses one of them uniformly at random. We have an error if there is no such pair, in which the encoder $f_{1}$ uniformly at random chooses $t_{1} \in \mathcal{B}\left(L_{0}, L_{1}\right)$, $t_{2} \in \mathcal{B}\left(L_{0}, L_{2}\right)$. The error probability of the last event approaches to zero as $n \rightarrow \infty$, if [36]

$$
\rho_{1}^{\prime}+\tilde{\rho}_{1}^{\prime} \leq \rho_{1}+\tilde{\rho}_{1}-\mathbb{I}\left(U_{1} ; U_{2} \mid U_{0}\right)-\epsilon_{1} .
$$

Finally, the encoder $f_{1}$ generates a sequence $X_{1}^{n}$ at random according to $\prod_{i=1}^{n} p\left(x_{1, i} \mid u_{0, i}, u_{1, i}, u_{2, i}\right)$. Encoder 2 proceeds similarly to encode $w_{2}$ and sends codeword $X_{2}^{n}$. The probability of not finding a jointly typical sequence pair $\left(v_{1}^{n}\left(L_{0}^{\prime}, s_{1}\left(L_{0}^{\prime}, L_{1}^{\prime}\right)\right), v_{2}^{n}\left(L_{0}^{\prime}, s_{2}\left(L_{0}^{\prime}, L_{1}^{\prime}\right)\right)\right) \in \mathcal{T}_{\epsilon_{2}}^{(n)}\left(V_{1}, V_{2} \mid V_{0}\right)$ in the product bin approaches to zero as $n \rightarrow \infty$, if [36]

$$
\rho_{2}^{\prime}+\tilde{\rho}_{2}^{\prime} \leq \rho_{2}+\tilde{\rho}_{2}-\mathbb{I}\left(V_{1} ; V_{2} \mid V_{0}\right)-\epsilon_{2} .
$$

\section{Decoding and Error Probability Analysis:}

- Let $\left(W_{1}, L_{0}, T_{1}\right)$ and $\left(W_{2}, L_{0}^{\prime}, S_{1}\right)$ denote the transmitted indices by the first and second transmitter, respectively, and let $\left(\hat{W}_{1}, \hat{L}_{0}, \hat{T}_{1}\right)$ and $\left(\hat{W}_{2}, \hat{L}_{0}^{\prime}, \hat{S}_{1}\right)$ denote the corresponding decoded messages by the first receiver, respectively. Receiver 1 decodes $\left(L_{0}, L_{0}^{\prime}\right)$ and therefore $\left(w_{1}, w_{2}\right)$ indirectly by finding a unique pair $\left(\hat{\ell}_{0}, \hat{\ell}_{0}^{\prime}\right)$ such that $\left(q^{n}, u_{0}^{n}\left(\hat{\ell}_{0}\right), u_{1}^{n}\left(\hat{\ell}_{0}, t_{1}\right), v_{0}^{n}\left(\hat{\ell}_{0}^{\prime}\right), v_{1}^{n}\left(\hat{\ell}_{0}^{\prime}, s_{1}\right), y_{1}^{n}\right) \in \mathcal{T}_{\epsilon^{\prime}}^{(n)}\left(U_{0}, U_{1}, V_{0}, V_{1}, Y_{1}\right)$ for some $t_{1} \in$ $\llbracket 1,2^{n \rho_{1}} \rrbracket$ and $s_{1} \in \llbracket 1,2^{n \rho_{2}} \rrbracket$. The idea of indirect decoding for the situation that there is just one transmitter is shown in Fig. 8 , The error event $\left(\hat{W}_{1}, \hat{W}_{1}\right) \neq\left(W_{1}, W_{1}\right)$ occurs only if at least one of the following events occurs:

$$
\begin{aligned}
& \mathcal{E}_{1}=\left\{\left(Q^{n}, U_{0}^{n}\left(\ell_{0}\right), U_{1}^{n}\left(\ell_{0}, t_{1}\right), V_{0}^{n}\left(\ell_{0}^{\prime}\right), V_{1}^{n}\left(\ell_{0}^{\prime}, s_{1}\right), Y_{1}^{n}\right) \notin \mathcal{T}_{\epsilon}^{(n)}\right\} \\
& \mathcal{E}_{2}=\left\{\left(Q^{n}, U_{0}^{n}\left(\hat{\ell}_{0}\right), U_{1}^{n}\left(\hat{\ell}_{0}, \hat{t}_{1}\right), V_{0}^{n}\left(\ell_{0}^{\prime}\right), V_{1}^{n}\left(\ell_{0}^{\prime}, s_{1}\right), Y_{1}^{n}\right) \in \mathcal{T}_{\epsilon}^{(n)} \text { for some } \hat{\ell}_{0} \neq \ell_{0}, \hat{t}_{1}\right\} \mathcal{E}_{3}=\left\{\left(Q^{n}, U_{0}^{n}\left(\ell_{0}\right), U_{1}^{n}\left(\ell_{0}, t_{1}\right), V_{0}^{n}\left(\hat{\ell}_{0}^{\prime}\right), V_{1}^{n}\left(\hat{\ell}_{0}^{\prime}, \hat{s}_{1}\right), Y_{1}^{n}\right) \in \mathcal{T}_{\epsilon}^{(n)} \text { for some } \hat{\ell}_{0}^{\prime} \neq \ell_{0}^{\prime}, \hat{s}_{1}\right\}, \\
& \mathcal{E}_{4}=\left\{\left(Q^{n}, U_{0}^{n}\left(\hat{\ell}_{0}\right), U_{1}^{n}\left(\hat{\ell}_{0}, \hat{t}_{1}\right), V_{0}^{n}\left(\ell_{0}^{\prime}\right), V_{1}^{n}\left(\ell_{0}^{\prime}, \hat{s}_{1}\right),\right.\right. \\
&\left.\left.Y_{1}^{n}\right) \in \mathcal{T}_{\epsilon}^{(n)} \text { for some } \hat{\ell}_{0} \neq \ell_{0}, \hat{t}_{1}, \hat{s}_{1} \neq s_{1}\right\}, \\
& \mathcal{E}_{5}=\left\{\left(Q^{n}, U_{0}^{n}\left(\ell_{0}\right), U_{1}^{n}\left(\ell_{0}, \hat{t}_{1}\right), V_{0}^{n}\left(\hat{\ell}_{0}^{\prime}\right), V_{1}^{n}\left(\hat{\ell}_{0}^{\prime}, \hat{s}_{1}\right),\right.\right.
\end{aligned}
$$




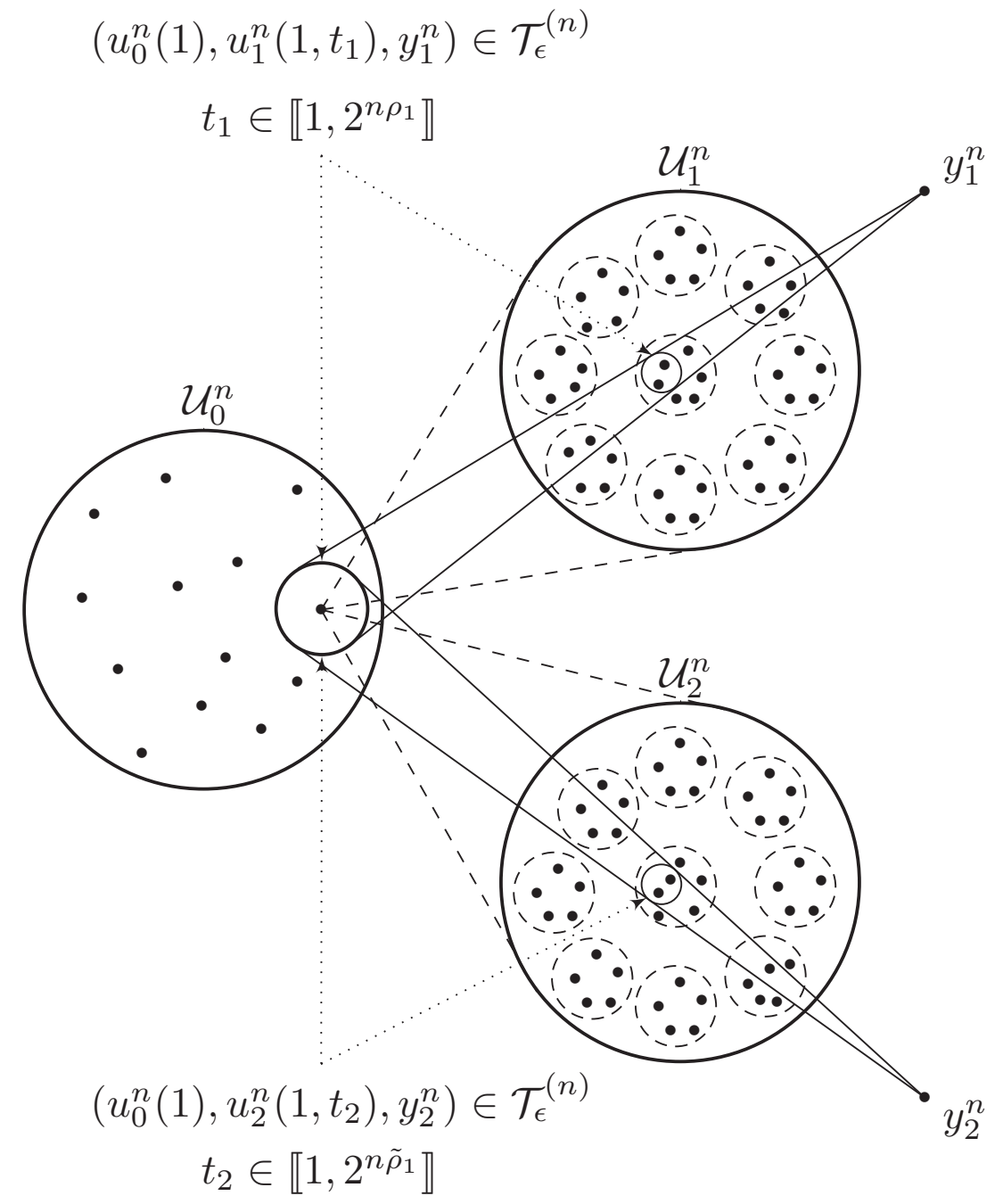

Fig. 8. Codebook structure and indirect decoding for $u_{0}^{n}(1)$ via $u_{1}^{n}\left(1, t_{1}\right)$ and $u_{2}^{n}\left(1, t_{2}\right)$ for the situation that there is just one transmitter.

$$
\begin{gathered}
\left.\left.Y_{1}^{n}\right) \in \mathcal{T}_{\epsilon}^{(n)} \text { for some } \hat{t}_{1} \neq t_{1}, \hat{\ell}_{0}^{\prime} \neq \ell_{0}^{\prime}, \hat{s}_{1}\right\}, \\
\mathcal{E}_{6}=\left\{\left(Q^{n}, U_{0}^{n}\left(\hat{\ell}_{0}\right), U_{1}^{n}\left(\hat{\ell}_{0}, \hat{t}_{1}\right), V_{0}^{n}\left(\hat{\ell}_{0}^{\prime}\right), V_{1}^{n}\left(\hat{\ell}_{0}^{\prime}, \hat{s}_{1}\right)\right.\right. \\
\left.\left., Y_{1}^{n}\right) \in \mathcal{T}_{\epsilon}^{(n)} \text { for some } \hat{\ell}_{0} \neq \ell_{0}, \hat{t}_{1}, \hat{\ell}_{0}^{\prime} \neq \ell_{0}^{\prime}, \hat{s}_{1}\right\} .
\end{gathered}
$$

Therefore, by Union Bound the average probability of error for decoder 1 is upper bounded as

$$
P_{e_{1}} \leq \mathbb{P}\left(\mathcal{E}_{1}\right)+\mathbb{P}\left(\mathcal{E}_{2}\right)+\mathbb{P}\left(\mathcal{E}_{3}\right)+\mathbb{P}\left(\mathcal{E}_{4}\right)+\mathbb{P}\left(\mathcal{E}_{5}\right)+\mathbb{P}\left(\mathcal{E}_{6}\right)
$$


By law of large numbers, $\mathbb{P}\left(\mathcal{E}_{1}\right)$ tends to zero as $n \rightarrow \infty$. By packing lemma [34, Lemma 3.1] $\mathbb{P}\left(\mathcal{E}_{2}\right)$ to $\mathbb{P}\left(\mathcal{E}_{6}\right)$ respectively tend to zero as $n \rightarrow \infty$ if

$$
\begin{array}{r}
\tilde{R}_{1}+\rho_{1}<\mathbb{I}\left(U_{0}, U_{1} ; Y_{1} \mid Q, V_{0}, V_{1}\right), \\
\tilde{R}_{2}+\rho_{2}<\mathbb{I}\left(V_{0}, V_{1} ; Y_{1} \mid Q, U_{0}, U_{1}\right), \\
\tilde{R}_{1}+\rho_{1}+\rho_{2}<\mathbb{I}\left(U_{0}, U_{1}, V_{1} ; Y_{1} \mid Q, V_{0}\right), \\
\rho_{1}+\tilde{R}_{2}+\rho_{2}<\mathbb{I}\left(U_{1}, V_{0}, V_{1} ; Y_{1} \mid Q, U_{0}\right), \\
\tilde{R}_{1}+\rho_{1}+\tilde{R}_{2}+\rho_{2}<\mathbb{I}\left(U_{0}, U_{1}, V_{0}, V_{1} ; Y_{1} \mid Q\right) .
\end{array}
$$

- Similarly Receiver 2 decodes $\left(L_{0}, L_{0}^{\prime}\right)$ and therefore $\left(w_{1}, w_{2}\right)$ indirectly by finding a unique pair $\left(\check{\ell}_{0}, \check{\ell}_{0}^{\prime}\right)$ such that $\left(q^{n}, u_{0}^{n}\left(\check{\ell}_{0}\right), u_{2}^{n}\left(\check{\ell}_{0}, t_{2}\right), v_{0}^{n}\left(\check{\ell}_{0}^{\prime}\right), v_{2}^{n}\left(\check{\ell}_{0}^{\prime}, s_{2}\right), y_{2}^{n}\right) \in \mathcal{T}_{\epsilon^{\prime \prime}}^{(n)}\left(U_{0}, U_{2}, V_{0}, V_{2}, Y_{2}\right)$ for some $t_{2} \in \llbracket 1,2^{n \tilde{\rho}_{1}} \rrbracket$ and $s_{2} \in \llbracket 1,2^{n \tilde{\rho}_{2}} \rrbracket$. The error analysis for the second receiver is similar to the first receiver and for the interest of brevity it is omitted here. Similar to Receiver 1 the The probability of error for Receiver 2 goes to zero as $n \rightarrow \infty$ if we choose [34]

$$
\begin{array}{r}
\tilde{R}_{1}+\tilde{\rho}_{1}<\mathbb{I}\left(U_{0}, U_{2} ; Y_{2} \mid Q, V_{0}, V_{2}\right), \\
\tilde{R}_{2}+\tilde{\rho}_{2}<\mathbb{I}\left(V_{0}, V_{2} ; Y_{2} \mid Q, U_{0}, U_{2}\right), \\
\tilde{R}_{1}+\tilde{\rho}_{1}+\tilde{\rho}_{2}<\mathbb{I}\left(U_{0}, U_{2}, V_{2} ; Y_{2} \mid Q, V_{0}\right), \\
\tilde{\rho}_{1}+\tilde{R}_{2}+\tilde{\rho}_{2}<\mathbb{I}\left(U_{2}, V_{0}, V_{2} ; Y_{2} \mid Q, U_{0}\right), \\
\tilde{R}_{1}+\tilde{\rho}_{1}+\tilde{R}_{2}+\tilde{\rho}_{2}<\mathbb{I}\left(U_{0}, U_{2}, V_{0}, V_{2} ; Y_{2} \mid Q\right) .
\end{array}
$$

Equivocation Calculation: We analyze mutual information between $\left(W_{1}, W_{2}\right)$ and $Z^{n}$, averaged over all random codebooks

$$
\begin{aligned}
& \mathbb{I}\left(W_{1}, W_{2} ; Z^{n} \mid Q^{n}, \mathcal{C}\right) \\
= & \mathbb{I}\left(W_{1}, W_{2}, L_{0}, T_{1}, T_{2}, L_{0}^{\prime}, S_{1}, S_{2} ; Z^{n} \mid Q^{n}, \mathcal{C}\right)-\mathbb{I}\left(L_{0}, T_{1}, T_{2}, L_{0}^{\prime}, S_{1}, S_{2} ; Z^{n} \mid W_{1}, W_{2}, Q^{n}, \mathcal{C}\right) \\
\leq & \mathbb{I}\left(U_{0}^{n}, U_{1}^{n}, U_{2}^{n}, V_{0}^{n}, V_{1}^{n}, V_{2}^{n} ; Z^{n} \mid Q^{n}, \mathcal{C}\right)-\mathbb{I}\left(L_{0}, L_{0}^{\prime} ; Z^{n} \mid W_{1}, W_{2}, Q^{n}, \mathcal{C}\right) \\
& \quad-\mathbb{I}\left(T_{1}, T_{2}, S_{1}, S_{2} ; Z^{n} \mid L_{0}, L_{0}^{\prime}, Q^{n}, \mathcal{C}\right) \\
= & \mathbb{I}\left(U_{0}^{n}, U_{1}^{n}, U_{2}^{n}, V_{0}^{n}, V_{1}^{n}, V_{2}^{n} ; Z^{n} \mid Q^{n}, \mathcal{C}\right)-\mathbb{H}\left(L_{0}, L_{0}^{\prime} \mid W_{1}, W_{2}, Q^{n}, \mathcal{C}\right) \\
& +\mathbb{H}\left(L_{0}, L_{0}^{\prime} \mid Z^{n}, W_{1}, W_{2}, Q^{n}, \mathcal{C}\right)-\mathbb{I}\left(T_{1}, T_{2}, S_{1}, S_{2} ; Z^{n} \mid L_{0}, L_{0}^{\prime}, Q^{n}, \mathcal{C}\right)
\end{aligned}
$$


where the inequality is due to the data processing inequality. Here, $T_{1}, T_{2}, S_{1}$, and $S_{2}$ are deterministic functions of $\left(L_{0}, L_{1}\right),\left(L_{0}, L_{2}\right),\left(L_{0}^{\prime}, L_{1}^{\prime}\right)$, and $\left(L_{0}^{\prime}, L_{2}^{\prime}\right)$, respectively.

The first term in (126) is bounded as:

$$
\mathbb{I}\left(U_{0}^{n}, U_{1}^{n}, U_{2}^{n}, V_{0}^{n}, V_{1}^{n}, V_{2}^{n} ; Z^{n} \mid Q^{n}, \mathcal{C}\right) \leq n \mathbb{I}\left(U_{0}, U_{1}, U_{2}, V_{0}, V_{1}, V_{2} ; Z \mid Q\right)+n \epsilon,
$$

as $n \rightarrow \infty$ where $\epsilon \rightarrow 0$ [34].

For the second term in (126) we have

$$
\mathbb{H}\left(L_{0}, L_{0}^{\prime} \mid W_{1}, W_{2}, Q^{n}, \mathcal{C}\right)=n\left(\tilde{R}_{1}-R_{1}+\tilde{R}_{2}-R_{2}\right)
$$

For the third term, substituting $U_{0} \leftarrow Q, V_{0} \leftarrow Q, U_{1} \leftarrow U_{0}$, and $V_{1} \leftarrow V_{0}$ in Lemma 1 result that,

$$
\mathbb{H}\left(L_{0}, L_{0}^{\prime} \mid Z^{n}, W_{1}, W_{2}, Q^{n}, \mathcal{C}\right) \leq n\left(\tilde{R}_{1}-R_{1}+\tilde{R}_{2}-R_{2}-\mathbb{I}\left(U_{0}, V_{0} ; Z \mid Q\right)+\epsilon\right),
$$

if $\mathbb{P}\left(\left(Q^{n}, U_{0}^{n}\left(L_{0}\right), V_{0}^{n}\left(L_{0}^{\prime}\right), Z^{n}\right) \in \mathcal{T}_{\epsilon}^{(n)}\right) \rightarrow 1$ as $n \rightarrow \infty$ and $\tilde{R}_{1}-R_{1} \geq \mathbb{I}\left(U_{0} ; Z \mid Q\right)+\epsilon$, $\tilde{R}_{2}-R_{2} \geq \mathbb{I}\left(V_{0} ; Z \mid Q\right)+\epsilon$, and $\tilde{R}_{1}-R_{1}+\tilde{R}_{2}-R_{2} \geq \mathbb{I}\left(U_{0}, V_{0} ; Z \mid Q\right)+\epsilon$.

Here, the first condition holds because

$$
\begin{aligned}
& \mathbb{P}\left(\left(Q^{n}, U_{0}^{n}\left(L_{0}\right), U_{1}^{n}\left(L_{0}, t_{1}\left(L_{0}, L_{1}\right)\right), U_{2}^{n}\left(L_{0}, t_{2}\left(L_{0}, L_{1}\right)\right), V_{0}^{n}\left(L_{0}^{\prime}\right), V_{1}^{n}\left(L_{0}^{\prime}, s_{1}\left(L_{0}^{\prime}, L_{1}^{\prime}\right)\right)\right.\right. \\
& \left.\left.\quad, V_{2}^{n}\left(L_{0}^{\prime}, s_{2}\left(L_{0}^{\prime}, L_{1}^{\prime}\right)\right), Z^{n}\right) \in \mathcal{T}_{\epsilon}^{(n)}\right) \rightarrow 1
\end{aligned}
$$

as $n \rightarrow \infty$. Now, we bound the last term in (126)

$$
\begin{aligned}
& \mathbb{I}\left(T_{1}, T_{2}, S_{1}, S_{2} ; Z^{n} \mid L_{0}, L_{0}^{\prime}, Q^{n}, \mathcal{C}\right) \\
& =\mathbb{H}\left(T_{1}, T_{2}, S_{1}, S_{2} \mid L_{0}, L_{0}^{\prime}, Q^{n}, \mathcal{C}\right)-\mathbb{H}\left(T_{1}, T_{2}, S_{1}, S_{2} \mid Z^{n}, L_{0}, L_{0}^{\prime}, Q^{n}, \mathcal{C}\right) \\
& \stackrel{(a)}{=} \mathbb{H}\left(T_{1}, T_{2}, S_{1}, S_{2}, L_{1}, L_{2}, L_{1}^{\prime}, L_{2}^{\prime} \mid L_{0}, L_{0}^{\prime}, Q^{n}, \mathcal{C}\right)-\mathbb{H}\left(T_{1}, T_{2}, S_{1}, S_{2} \mid Z^{n}, L_{0}, L_{0}^{\prime}, Q^{n}, \mathcal{C}\right) \\
& \geq \mathbb{H}\left(L_{1}, L_{2}, L_{1}^{\prime}, L_{2}^{\prime} \mid L_{0}, L_{0}^{\prime}, Q^{n}, \mathcal{C}\right)-\mathbb{H}\left(T_{1}, S_{1} \mid Z^{n}, L_{0}, L_{0}^{\prime}, Q^{n}, \mathcal{C}\right)-\mathbb{H}\left(T_{2}, S_{2} \mid Z^{n}, L_{0}, L_{0}^{\prime}, Q^{n}, \mathcal{C}\right) \\
& \stackrel{(b)}{=} \mathbb{H}\left(L_{1}, L_{2} \mid L_{0}, L_{0}^{\prime}, Q^{n}, \mathcal{C}\right)+\mathbb{H}\left(L_{1}^{\prime}, L_{2}^{\prime} \mid L_{0}, L_{0}^{\prime}, Q^{n}, \mathcal{C}\right) \\
& -\mathbb{H}\left(T_{1}, S_{1} \mid Z^{n}, L_{0}, L_{0}^{\prime}, Q^{n}, \mathcal{C}\right)-\mathbb{H}\left(T_{2}, S_{2} \mid Z^{n}, L_{0}, L_{0}^{\prime}, Q^{n}, \mathcal{C}\right),
\end{aligned}
$$

where $(a)$ is due to given the codebook $\mathcal{C}$ and $\left(L_{0}, L_{0}^{\prime}\right),\left(L_{1}, L_{2}, L_{1}^{\prime}, L_{2}^{\prime}\right)$ is a deterministic function of $\left(T_{1}\left(L_{0}, L_{1}\right), T_{2}\left(L_{0}, L_{2}\right), S_{1}\left(L_{0}^{\prime}, L_{1}^{\prime}\right), S_{2}\left(L_{0}^{\prime}, L_{2}^{\prime}\right)\right)$, and $(b)$ holds due to the fact that given $\left(L_{0}, L_{0}^{\prime}, Q^{n}, \mathcal{C}\right),\left(L_{1}, L_{2}\right)$ and $\left(L_{1}^{\prime}, L_{2}^{\prime}\right)$ are independent. Now,

$$
\mathbb{H}\left(L_{1}, L_{2} \mid L_{0}, L_{0}^{\prime}, Q^{n}, \mathcal{C}\right)=n\left(\rho_{1}^{\prime}+\tilde{\rho}_{1}^{\prime}\right)
$$




$$
\begin{aligned}
& \mathbb{H}\left(L_{1}^{\prime}, L_{2}^{\prime} \mid L_{0}, L_{0}^{\prime}, Q^{n}, \mathcal{C}\right)=n\left(\rho_{2}^{\prime}+\tilde{\rho}_{2}^{\prime}\right), \\
& \mathbb{H}\left(T_{1}, S_{1} \mid Z^{n}, L_{0}, L_{0}^{\prime}, Q^{n}, \mathcal{C}\right) \stackrel{(a)}{\leq} n\left(\rho_{1}+\rho_{2}-\mathbb{I}\left(U_{1}, V_{1} ; Z \mid Q, U_{0}, V_{0}\right)+\epsilon\right), \\
& \mathbb{H}\left(T_{2}, S_{2} \mid Z^{n}, L_{0}, L_{0}^{\prime}, Q^{n}, \mathcal{C}\right) \stackrel{(b)}{\leq} n\left(\tilde{\rho}_{1}+\tilde{\rho}_{2}-\mathbb{I}\left(U_{2}, V_{2} ; Z \mid Q, U_{0}, V_{0}\right)+\epsilon\right),
\end{aligned}
$$

where $(a)$ is due to the following. Consider,

$$
\begin{aligned}
& \mathbb{H}\left(T_{1}, S_{1} \mid Z^{n}, L_{0}, L_{0}^{\prime}, Q^{n}, \mathcal{C}\right)=\mathbb{H}\left(T_{1}, S_{1} \mid U_{0}^{n}\left(L_{0}\right), V_{0}^{n}\left(L_{0}^{\prime}\right), Z^{n}, L_{0}, L_{0}^{\prime}, Q^{n}, \mathcal{C}\right) \\
& \quad \leq \mathbb{H}\left(T_{1}, S_{1} \mid U_{0}^{n}\left(L_{0}\right), V_{0}^{n}\left(L_{0}^{\prime}\right), Z^{n}, Q^{n}, \mathcal{C}\right)
\end{aligned}
$$

Now we upper bound the term $\mathbb{H}\left(T_{1}, S_{1} \mid U_{0}^{n}\left(L_{0}\right), V_{0}^{n}\left(L_{0}^{\prime}\right), Z^{n}, Q^{n}, \mathcal{C}\right)$. From (130) we have $\mathbb{P}\left(\left(Q^{n}, U_{0}^{n}\left(L_{0}\right), U_{1}^{n}\left(L_{0}, t_{1}\left(L_{0}, L_{1}\right)\right), V_{0}^{n}\left(L_{0}^{\prime}\right), V_{1}^{n}\left(L_{0}^{\prime}, s_{1}\left(L_{0}^{\prime}, L_{1}^{\prime}\right)\right), Z^{n}\right) \in \mathcal{T}_{\epsilon}^{(n)}\right) \rightarrow 1$ as $n \rightarrow \infty$. Applying Lemma 1 leads to,

$$
\mathbb{H}\left(T_{1}, S_{1} \mid U_{0}^{n}\left(L_{0}\right), V_{0}^{n}\left(L_{0}^{\prime}\right), Z^{n}, Q^{n}, \mathcal{C}\right) \leq n\left(\rho_{1}+\rho_{2}-\mathbb{I}\left(U_{1}, V_{1} ; Z \mid Q, U_{0}, V_{0}\right)+\epsilon\right),
$$

if $\rho_{1} \geq \mathbb{I}\left(U_{1} ; Z \mid Q, U_{0}, V_{0}\right)+\epsilon, \rho_{2} \geq \mathbb{I}\left(V_{1} ; Z \mid Q, U_{0}, V_{0}\right)+\epsilon$, and $\rho_{1}+\rho_{2} \geq \mathbb{I}\left(U_{1}, V_{1} ; Z \mid Q, U_{0}, V_{0}\right)+\epsilon$. By the same argument the inequality $(b)$ holds, if the following inequalities hold,

$$
\begin{gathered}
\tilde{\rho}_{1} \geq \mathbb{I}\left(U_{2} ; Z \mid Q, U_{0}, V_{0}\right)+\epsilon, \\
\tilde{\rho}_{2} \geq \mathbb{I}\left(V_{2} ; Z \mid Q, U_{0}, V_{0}\right)+\epsilon, \\
\tilde{\rho}_{1}+\tilde{\rho}_{2} \geq \mathbb{I}\left(U_{2}, V_{2} ; Z \mid Q, U_{0}, V_{0}\right)+\epsilon,
\end{gathered}
$$

Substituting (132)-135) into (131) leads to,

$$
\begin{aligned}
& \mathbb{I}\left(T_{1}, T_{2}, S_{1}, S_{2} ; Z^{n} \mid L_{0}, L_{0}^{\prime}, Q^{n}, \mathcal{C}\right) \\
& \geq n\left(\rho_{1}^{\prime}+\tilde{\rho}_{1}^{\prime}\right)+n\left(\rho_{2}^{\prime}+\tilde{\rho}_{2}^{\prime}\right)-n\left(\rho_{1}+\rho_{2}-\mathbb{I}\left(U_{1}, V_{1} ; Z \mid Q, U_{0}, V_{0}\right)+\epsilon\right) \\
& \quad-n\left(\tilde{\rho}_{1}+\tilde{\rho}_{2}-\mathbb{I}\left(U_{2}, V_{2} ; Z \mid Q, U_{0}, V_{0}\right)+\epsilon\right) .
\end{aligned}
$$

Substituting (127)-(129) and (137) into (126) yields

$$
\begin{aligned}
& \mathbb{I}\left(W_{1}, W_{2} ; Z^{n} \mid Q^{n}, \mathcal{C}\right) \leq n \mathbb{I}\left(U_{0}, U_{1}, U_{2}, V_{0}, V_{1}, V_{2} ; Z \mid Q\right)-n\left(\tilde{R}_{1}-R_{1}+\tilde{R}_{2}-R_{2}\right) \\
& +n\left(\tilde{R}_{1}-R_{1}+\tilde{R}_{2}-R_{2}-\mathbb{I}\left(U_{0}, V_{0} ; Z \mid Q\right)\right)-n\left(\rho_{1}^{\prime}+\tilde{\rho}_{1}^{\prime}\right)-n\left(\rho_{2}^{\prime}+\tilde{\rho}_{2}^{\prime}\right) \\
& +n\left(\rho_{1}+\rho_{2}-\mathbb{I}\left(U_{1}, V_{1} ; Z \mid Q, U_{0}, V_{0}\right)+\epsilon\right)+n\left(\tilde{\rho}_{1}+\tilde{\rho}_{2}-\mathbb{I}\left(U_{2}, V_{2} ; Z \mid Q, U_{0}, V_{0}\right)+\epsilon\right) .
\end{aligned}
$$

Therefore $\mathbb{I}\left(W_{1}, W_{2} ; Z^{n} \mid Q^{n}, \mathcal{C}\right) \leq n \epsilon$ if

$$
\mathbb{I}\left(U_{1}, U_{2}, V_{1}, V_{2} ; Z \mid U_{0}, V_{0}\right)-\rho_{1}^{\prime}-\tilde{\rho}_{1}^{\prime}-\rho_{2}^{\prime}-\tilde{\rho}_{2}^{\prime}+\rho_{1}+\rho_{2}-\mathbb{I}\left(U_{1}, V_{1} ; Z \mid Q, U_{0}, V_{0}\right)
$$




$$
+\tilde{\rho}_{1}+\tilde{\rho}_{2}-\mathbb{I}\left(U_{2}, V_{2} ; Z \mid Q, U_{0}, V_{0}\right) \leq \epsilon .
$$

As a result, the rate constraints derived in equivocation analysis are

$$
\begin{aligned}
& \tilde{R}_{1}-R_{1}+\tilde{R}_{2}-R_{2} \geq \mathbb{I}\left(U_{0}, V_{0} ; Z \mid Q\right) \\
& \tilde{R}_{1}-R_{1} \geq \mathbb{I}\left(U_{0} ; Z \mid Q\right) \\
& \tilde{R}_{2}-R_{2} \geq \mathbb{I}\left(V_{0} ; Z \mid Q\right) \\
& \rho_{1}+\rho_{2} \geq \mathbb{I}\left(U_{1}, V_{1} ; Z \mid Q, U_{0}, V_{0}\right) \\
& \rho_{1} \geq \mathbb{I}\left(U_{1} ; Z \mid Q, U_{0}, V_{0}\right) \\
& \rho_{2} \geq \mathbb{I}\left(V_{1} ; Z \mid Q, U_{0}, V_{0}\right) \\
& \tilde{\rho}_{1}+\tilde{\rho}_{2} \geq \mathbb{I}\left(U_{2}, V_{2} ; Z \mid Q, U_{0}, V_{0}\right) \\
& \tilde{\rho}_{1} \geq \mathbb{I}\left(U_{2} ; Z \mid Q, U_{0}, V_{0}\right) \\
& \tilde{\rho}_{2} \geq \mathbb{I}\left(V_{2} ; Z \mid Q, U_{0}, V_{0}\right) \\
& \rho_{1}+\rho_{2}+\tilde{\rho}_{1}+\tilde{\rho}_{2}-\rho_{1}^{\prime}-\tilde{\rho}_{1}^{\prime}-\rho_{2}^{\prime}-\tilde{\rho}_{2}^{\prime} \leq \mathbb{I}\left(U_{1}, V_{1} ; Z \mid Q, U_{0}, V_{0}\right)+\mathbb{I}\left(U_{2}, V_{2} ; Z \mid Q, U_{0}, V_{0}\right) \\
& \quad-\mathbb{I}\left(U_{1}, U_{2}, V_{1}, V_{2} ; Z \mid U_{0}, V_{0}\right)
\end{aligned}
$$

Finally, by applying the Fourier-Motzkin procedure [33] to (108), (109), (116)-(125), and (140)(149) we obtain the inequalities in Theorem 1 .

\section{ACKNOWLEDGMENT}

The authors would like to thank anonymous reviewers of ISIT 2018 for their helpful comments.

\section{REFERENCES}

[1] A. Cohen, A. Cohen, M. Médard, and O. Gurewitz, "Secure multi-source multicast," available at https://arxiv.org/abs/1702.03012, Nov. 2017.

[2] O. Tervo, L.-M. Tran, H. Pennanen, S. Chatzinotas, B. Ottersten, and M. Juntti, "Energy-efficient multi-cell multigroup multicasting with joint beamforming and antenna selection," available at https://arxiv.org/abs/1808.00852. Aug. 2018.

[3] Y. Liang, G. Kramer, H. V. Poor, and S. Shamai, "Compound wiretap channels," in Proc. 45th Annual Allerton Conference on Communication, Control, and Computing, Monticello,IL, Sep. 2007, pp. 136-143.

[4] R. Ahlswede, "The capacity region of a channel with two senders and two receivers," Ann. Probab., vol. 2, no. 5, pp. 805-814, 1974.

[5] S. Verdú, "Fifty years of Shannon theory," IEEE Trans. Inf. Theory, vol. 44, no. 6, pp. 2057-2078, Oct. 1998. 
[6] T. S. Han and K. Kobayashi, "A new achievable rate region for the interference channel," IEEE Trans. Inf. Theory, vol. 27, no. 1, pp. 49-60, Jan. 1981.

[7] Y. K. Chia and A. El Gamal, "Three-receiver broadcast channel with common and confidential messages," IEEE Trans. Inf. Theory, vol. 58, no. 9, pp. 2748-2765, May 2012.

[8] C. Nair and A. El Gamal, "The capacity region of a class of three-receiver broadcast channels with degraded message sets," IEEE Trans. Inf. Theory, vol. 55, no. 10, pp. 4479-4493, Oct. 2009.

[9] M. H. Yassaee, M. R. Aref, and A. A. Gohari, "Achievability proof via output statistics of random binning," IEEE Trans. Inf. Theory, vol. 60, no. 11, pp. 6786-6760, Nov. 2014.

[10] J. M. Renes and R. Renner, "Noisy channel coding via privacy amplification and information reconciliation," IEEE Trans. Inf. Theory, vol. 57, no. 11, pp. 7377-7385, Nov. 2011.

[11] I. Csiszár, "Almost independence and secrecy capacity," IEEE Trans. Inf. Theory, vol. 32, no. 1, pp. 40-47, Jan. 1996.

[12] S. Watanabe and Y. Oohama, "The optimal use of rate-limited randomness in broadcast channels with confidential messages," IEEE Trans. Inf. Theory, vol. 61, no. 2, pp. 983-995, Feb 2015.

[13] H. ZivariFard, M. R. Bloch, and A. Nosratinia, “Two-transmitter two-receiver channel with confidential messages," in Proc. 55th Annual Allerton Conference on Communication, Control, and Computing, Monticello,IL, Sep. 2017, pp. $103-110$.

[14] S. Salehkalaibar, M. Mirmohseni, and M. R. Aref, "One-receiver two-eavesdropper broadcast channel with degraded message sets," IEEE Trans. Inf. Forensics Security, vol. 8, no. 12, pp. 1961-1974, Dec. 2013.

[15] E. Ekrem and S. Ulukus, "Multi-receiver wiretap channel with public and confidential messages," IEEE Trans. Inf. Theory, vol. 59, no. 4, pp. 2165-2177, Apr. 2013.

[16] M. Benammar and P. Piantanida, "Secrecy capacity region of some classes of wiretap broadcast channels," IEEE Trans. Inf. Theory, vol. 61, no. 10, pp. 5564-5582, Oct. 2015.

[17] L. Liang and H. V. Poor, "Multiple-access channels with confidential messages," IEEE Trans. Inf. Theory, vol. 3, no. 3, pp. 976-1002, Mar. 2008.

[18] E. Tekin and A. Yener, "The Gaussian multiple access wire-tap channel," IEEE Trans. Inf. Theory, vol. 54, no. 12, pp. 5747-5755, Dec. 2008.

[19] _ - "The general Gaussian multiple-access and two-way wiretap channels: Achievable rates and cooperative jamming," IEEE Trans. Inf. Theory, vol. 54, no. 6, pp. 2735-2751, Jun. 2008.

[20] M. H. Yassaee and M. R. Aref, "Multiple access wiretap channels with strong secrecy," in Proc. IEEE Info. Theory Workshop (ITW), Dublin, Ireland, Sep. 2010, pp. 1-5.

[21] M. Wiese and H. Boche, "Strong secrecy for multiple access channels," in Information Theory, Combinatorics, and Search Theory, Springer, 2013, pp. 71-122.

[22] A. J. Pierrot and M. R. Bloch, "Strongly secure communications over the two-way wiretap channel," IEEE Trans. Inf. Forensics Security, vol. 6, no. 3, pp. 595-605, Sep. 2011.

[23] H. Zivari-Fard, B. Akhbari, M. Ahmadian-Attari, and M. R. Aref, "Imperfect and perfect secrecy in compound multiple access channel with confidential message,” IEEE Trans. Inf. Forensics Security, vol. 11, no. 6, pp. 1239 -1251, Jun. 2016.

[24] A. A. Chou and A. Yener, "Polar coding for the multiple access wiretap channel via rate-splitting and cooperative jamming," IEEE Trans. Inf. Theory, vol. 64, no. 12, pp. 7903-7921, Dec. 2018.

[25] A. Carleial, "Multiple-access channels with different generalized feedback signals," IEEE Trans. Inf. Theory, vol. 28, no. 6, pp. 841-850, Nov. 1982.

[26] R. Liu, I. Marić, P. Spasojević, and R. D. Yates, "Discrete memoryless interference and broadcast channels with confidential messages," IEEE Trans. Inf. Theory, vol. 54, no. 6, pp. 2493-2507, Jun. 2008.

[27] P. Cuff, "Distributed channel synthesis," IEEE Trans. Inf. Theory, vol. 59, no. 11, pp. 7071-7096, Nov. 2013. 
[28] M. R. Bloch and J. Barros, Physical-Layer Security: From Information Theory to Security Engineering, 1st ed. Cambridge, U.K: Cambridge University Press, 2011.

[29] Y. Liang, H. V. Poor, and S. Shamai, Information Theoretic Security, 1st ed. Hanover, MA, USA: Now Publishers Inc., 2009.

[30] I. Csiszár and J. Körner, "Broadcast channels with confidential messages," IEEE Trans. Inf. Theory, vol. 24, no. 3, pp. 339-348, May 1978.

[31] I. Csiszár and J. Körner, "Broadcast channels with confidential messages," IEEE Trans. Inf. Theory, vol. 24, no. 3, pp. 339-348, May 1978.

[32] I. B. Gattegno, Z. Goldfeld, and H. H. Permuter, "Fourier-Motzkin elimination software for information theoretic inequalities," in http://www.ee.bgu.ac.il/ fmeit/, 2016.

[33] F. S. Chaharsooghi, M. J. Emadi, M. Zamanighomi, and M. R. Aref, "A new method for variable elimination in systems of inequations," in Proc. IEEE Int. Symp. on Info. Theory (ISIT), St. Petersburg, Russia, Jul. 2011, pp. 1215-1219.

[34] A. El Gamal and Y.-H. Kim, Network Information Theory, 1st ed. Cambridge, U.K: Cambridge University Press, 2012.

[35] A. D. Wyner, “The wire-tap channel,” Bell System Technical Journal, vol. 57, no. 8, pp. 1355-1367, Oct. 1975.

[36] A. El Gamal and E. C. van der Meulen, "A proof of Marton's coding theorem for the discrete memoryless broadcast channel," IEEE Trans. Inf. Theory, vol. 27, no. 1, pp. 120-122, Jan. 1981. 